TRANSACTIONS OF THE

AMERICAN MATHEMATICAL SOCIETY

Volume 349, Number 8, August 1997, Pages 3181-3234

S 0002-9947(97)01963-6

\title{
AN INFINITE DIMENSIONAL MORSE THEORY WITH APPLICATIONS
}

\author{
WOJCIECH KRYSZEWSKI AND ANDRZEJ SZULKIN
}

\begin{abstract}
In this paper we construct an infinite dimensional (extraordinary) cohomology theory and a Morse theory corresponding to it. These theories have some special properties which make them useful in the study of critical points of strongly indefinite functionals (by strongly indefinite we mean a functional unbounded from below and from above on any subspace of finite codimension). Several applications are given to Hamiltonian systems, the onedimensional wave equation (of vibrating string type) and systems of elliptic partial differential equations.
\end{abstract}

\section{INTRODUCTION}

Let $E$ be a real Hilbert space with an inner product $\langle.,$.$\rangle and let \Phi$ be a twice continuously differentiable functional. Denote the Fréchet derivative and the gradient of $\Phi$ at $x$ by $\Phi^{\prime}(x)$ and $\nabla \Phi(x)$ respectively, where as usual

$$
\langle\nabla \Phi(x), y\rangle:=\Phi^{\prime}(x) y \quad \forall y \in E .
$$

Recall that a point $x_{0} \in E$ is said to be critical if $\Phi^{\prime}\left(x_{0}\right)=0$, or equivalently, if $\nabla \Phi\left(x_{0}\right)=0$. The level $c \in \mathbf{R}$ will be called regular if $\Phi^{-1}(c)$ contains no critical points, and critical if $\nabla \Phi\left(x_{0}\right)=0$ for some $x_{0} \in \Phi^{-1}(c)$.

Let $a, b, a<b$, be two regular levels of $\Phi$. Denote $M:=\Phi^{-1}([a, b])$ and consider the restriction of $\Phi$ to $M$. In Morse theory one is interested in the local topological structure of the level sets of $\left.\Phi\right|_{M}$ near a critical point and in the relation between this local structure and the topological structure of the set $M$. To be more specific, suppose that $x_{0} \in M$ is an isolated critical point of $\Phi$. Then one defines a sequence of critical groups of $\Phi$ at $x_{0}$ by setting

$$
c_{q}\left(\Phi, x_{0}\right):=H_{q}\left(\Phi^{c} \cap U, \Phi^{c} \cap U-\left\{x_{0}\right\}\right), \quad q=0,1,2, \ldots,
$$

where $c:=\Phi\left(x_{0}\right), \Phi^{c}:=\{x \in E: \Phi(x) \leq c\}, H_{q}$ is the $q$-th singular homology group with coefficients in some field $\mathcal{F}$ and $U$ is a neighbourhood of $x_{0}$. Define the Morse index of $x_{0}$ to be the maximal dimension of a subspace of $E$ on which the quadratic form $\left\langle\Phi^{\prime \prime}\left(x_{0}\right) y, y\right\rangle$ is negative definite. One shows that if $x_{0}$ is a nondegenerate critical point, i.e., if $\Phi^{\prime \prime}\left(x_{0}\right): E \rightarrow E$ is invertible, then $c_{q}\left(\Phi, x_{0}\right)=\mathcal{F}$

Received by the editors March 20, 1995.

1991 Mathematics Subject Classification. Primary 58E05; Secondary 34C25, 35J65, 35L05, $55 \mathrm{~N} 20,58 \mathrm{~F} 05$.

Key words and phrases. Filtration, cohomology, critical group, Morse inequalities, Morse index, degree theory, Hamiltonian system, wave equation, system of elliptic partial differential equations.

The first author was supported in part by the KBN Grant PB 513/2/91.

The second author was supported in part by the Swedish Natural Science Research Council. 
for $q=$ the Morse index of $x_{0}$, and $c_{q}\left(\Phi, x_{0}\right)=0$ otherwise. So in this case the groups $c_{q}\left(\Phi, x_{0}\right)$ are uniquely determined by the Morse index. If $x_{0}$ is degenerate, no such simple relation exists. The global aspect of Morse theory is expressed by the Morse inequalities which relate the critical groups of all critical points of $\left.\Phi\right|_{M}$ to the homology groups $H_{q}\left(\Phi^{b}, \Phi^{a}\right)$ (which are isomorphic with $H_{q}\left(M, \Phi^{-1}(a)\right.$ ) by the excision property of homology).

In what follows we assume that the reader is somewhat familiar with Morse theory in Hilbert spaces. Necessary prerequisites (and much more!) may be found e.g. in Chang [10] and Mawhin and Willem [34]. Let us also mention that a different approach to Morse theory, based on the Conley index, has been developed by Benci (see [6] and the references there).

The purpose of this paper is to construct a Morse theory for strongly indefinite functionals. In order to explain why the ordinary theory fails in this case, let us consider the following very simple example: Let $E=E^{+} \oplus E^{-}$be an orthogonal decomposition of $E$ into two infinite dimensional subspaces and suppose $\Phi(x)=$ $\frac{1}{2}\left\|x^{+}\right\|^{2}-\frac{1}{2}\left\|x^{-}\right\|^{2}$, where $x^{+} \in E^{+}$and $x^{-} \in E^{-}$. Then 0 is the only critical point of $\Phi$; it is nondegenerate and has the Morse index $+\infty$. Therefore $c_{q}(\Phi, 0)=0$ for all $q$ (this can also be easily computed directly from the definition of $c_{q}$ ). More generally, if $\Phi(x)=\frac{1}{2}\left\|x^{+}\right\|^{2}-\frac{1}{2}\left\|x^{-}\right\|^{2}+\psi(x)$, where $\nabla \psi$ is a compact mapping (i.e., it takes bounded sets to precompact ones), then the Morse index of any critical point must necessarily be $+\infty$. So in this case one cannot expect to obtain any useful information from the usual Morse theory.

In order to remedy this difficulty, the second author has introduced a different Morse theory in [41]. It was based on a suitably adapted version of an infinite dimensional cohomology theory due to Gȩba and Granas [20, 21]. In the present paper we construct another infinite dimensional cohomology theory and a Morse theory associated with it. Let $\left(E_{n}\right)_{n=1}^{\infty}$ be a filtration of $E$, i.e., an increasing sequence of closed subspaces of $E$ such that $E=\operatorname{cl}\left(\bigcup_{n=1}^{\infty} E_{n}\right)$ (cl denotes the closure), and let $\mathcal{E}=\left\{E_{n}, d_{n}\right\}_{n=1}^{\infty}$, where $\left(d_{n}\right)_{n=1}^{\infty}$ is a sequence of nonnegative integers. Then for a pair $(X, A)$ of closed sets in $E, A \subset X$, we define cohomology groups of $(X, A)$ by setting

$$
H_{\mathcal{E}}^{q}(X, A):=\left\{H^{q+d_{n}}\left(X \cap E_{n}, A \cap E_{n}\right)\right\}_{n=1}^{\infty}, \quad q \in \mathbf{Z} .
$$

Since we are only interested in the asymptotic behaviour of the sequences on the right-hand side above, we will consider $\left(\xi_{n}\right)_{n=1}^{\infty}$ and $\left(\eta_{n}\right)_{n=1}^{\infty}$, where $\xi_{n}, \eta_{n} \in$ $H^{q+d_{n}}\left(X \cap E_{n}, A \cap E_{n}\right)$, as equivalent (or representing the same element of $H_{\mathcal{E}}^{q}(X, A)$ ) if $\xi_{n}=\eta_{n}$ for almost all $n$. In applications $E_{n}$ will be a direct summand of $E_{n+1}$, and we will have $\operatorname{dim}\left(E_{n+1} \ominus E_{n}\right)=k$ and $d_{n}=c+d n$, where $0<d \leq k$ and the constants $c, d, k$ are independent of $n$.

In [41] the cohomology groups were obtained as the limit as $n \rightarrow \infty$ of the direct system $\left\{H^{q+n}\left(X \cap E_{n}, A \cap E_{n}\right), \Delta_{n}^{q}\right\}$, where $\left(E_{n}\right)_{n=1}^{\infty}$ is a filtration with $\operatorname{dim}\left(E_{n+1} \ominus E_{n}\right)=1$ and $\Delta_{n}^{q}: H^{q+n}\left(X \cap E_{n}, A \cap E_{n}\right) \rightarrow H^{q+n+1}\left(X \cap E_{n+1}, A \cap E_{n+1}\right)$ is a certain Mayer-Vietoris homomorphism. The objects we construct here are more unusual: each group $H_{\mathcal{E}}^{q}(X, A)$ is in fact a sequence of cohomology groups of the spaces $\left(X \cap E_{n}, A \cap E_{n}\right)$ approximating $(X, A)$. Although this is a certain disadvantage compared to [41], our approach here has advantages which prevail. It is much more flexible and more elementary. In particular, the fact that we may have $\operatorname{dim}\left(E_{n+1} \ominus E_{n}\right)>1$ and $d_{n+1}-d_{n}>1$ will turn out to be very convenient in applications. E.g., for Hamiltonian systems in $\mathbf{R}^{2 N}$ the natural choice of $E_{n}$ and $d_{n}$ 
is such that $\operatorname{dim}\left(E_{n+1} \ominus E_{n}\right)=4 N$ and $d_{n}=N(1+2 n)\left(E_{n}\right.$ is in fact the space of $n$ th partial sums in the Fourier expansion of functions in $E$, so $\operatorname{dim} E_{n}=2 N(1+2 n)$ ). By this choice it will be possible to avoid a tedious approximation procedure which was needed in [41, Section 6]. Furthermore, if the functional $\Phi$ is invariant with respect to an action of a group $\mathcal{G}$, then in most cases $E_{n+1} \ominus E_{n}$ cannot be onedimensional if it is to be $\mathcal{G}$-invariant.

The cohomology theory (0.2) will be constructed in Section 1, and in Sections 25 we construct a corresponding Morse theory. We use an approach due to Gromoll and Meyer $[10,22]$ and combine it with some ideas from the Conley index theory $[6,11]$ (note that our cohomology is defined only on pairs of closed sets, so critical groups cannot be introduced by a formula similar to (0.1)). Let us point out that the functionals considered in [41] satisfied the Palais-Smale condition (PS) and were of the form $\Phi(x)=\frac{1}{2}\langle L x, x\rangle+\psi(x)$, with $L$ linear and $P_{F} \nabla \psi$ compact $\left(P_{F}\right.$ is the orthogonal projector onto a certain subspace of $E$ ). Here we assume that $\Phi$ satisfies the condition (PS)*, which is somewhat stronger than (PS) but makes any further assumptions on the form of $\Phi$ unnecessary. In Section 6 a degree theory is constructed. It is related to our Morse theory via formulas of Poincaré-Hopf type.

The remaining sections are devoted to applications. In Section 7 we consider the problem of existence of periodic solutions for a Hamiltonian system of differential equations

$$
\dot{z}=J H_{z}(z, t)
$$

with Hamiltonian $H$ asymptotically quadratic at 0 and infinity. We extend several earlier results; see Remark 7.12 for more comments on previous work.

In Section 8 we are concerned with the asymptotically linear wave equation

$$
u_{t t}-u_{x x}=f(x, t, u)
$$

satisfying the boundary and the periodicity conditions

$$
u(0, t)=u(\pi, t)=0, \quad u(x, t+2 \pi)=u(x, t) .
$$

We extend earlier results contained in $[1,27]$ (cf. Remark 8.7). Moreover, we work directly with the functional

$$
\Phi(u):=\frac{1}{2} \int_{\Omega}\left(u_{t}^{2}-u_{x}^{2}\right) d x d t+\int_{\Omega} F(x, t, u) d x d t
$$

(where $\Omega=(0, \pi) \times(0,2 \pi)$ and $F$ is the primitive of $f$ ) which is natural for this problem. This is in fact one of the main advantages of our approach. In [1] a finite dimensional reduction was performed, and for this purpose it was necessary to assume that the derivative $f_{u}$ of $f$ is bounded (here $f$ need not be differentiable). The gradient $\nabla \psi$ of the non-quadratic part of $\Phi$ does not satisfy the previously mentioned compactness condition. Therefore the theory of [41] cannot be applied directly. Still, in [27] this theory was used, but only after introducing a different functional - which had all properties required in [41].

Section 9 is concerned with the system of elliptic partial differential equations

$$
-\Delta v=F_{u}(x, u, v), \quad-\Delta u=F_{v}(x, u, v),\left.\quad u\right|_{\partial \Omega}=\left.v\right|_{\partial \Omega}=0
$$

in a bounded domain $\Omega \subset \mathbf{R}^{N}$. Note that the functional

$$
\Phi(u, v):=\int_{\Omega} \nabla u \cdot \nabla v d x-\int_{\Omega} F(x, u, v) d x
$$

corresponding to this problem is strongly indefinite. 
Notation and terminology. The closure of a set $X$ will be denoted by $\bar{X} \operatorname{or} \operatorname{cl}(X)$, the interior by $\operatorname{int}(X)$ and the boundary by $\partial X . B(p, r)$ is the open ball, $\bar{B}(p, r)$ the closed ball and $S(p, r)$ the sphere of radius $r$ centered at $p$. Two sets $A, B$ are said to be bounded away from each other if $d(A, B):=\inf \{\|x-y\|: x \in A, y \in B\}>0$. An increasing sequence $\left(E_{n}\right)_{n=1}^{\infty}$ of closed subspaces of a Hilbert space $E$ is called a filtration of $E$ if $E=\operatorname{cl}\left(\bigcup_{n=1}^{\infty} E_{n}\right)$. A filtration together with a given sequence $\left(d_{n}\right)_{n=1}^{\infty}$ of nonnegative integers will be denoted by $\mathcal{E}=\left\{E_{n}, d_{n}\right\}_{n=1}^{\infty}$ (somewhat improperly, we will sometimes write $\mathcal{E}=\left(E_{n}\right)_{n=1}^{\infty}$ when the choice of the numbers $d_{n}$ is immaterial). A continuous function is called a mapping, and a mapping $f: X \rightarrow Y$ (or $f:(X, A) \rightarrow(Y, B)$ ), where $X, Y \subset E$, is filtration-preserving if $f\left(X \cap E_{n}\right) \subset E_{n}$ for almost all $n$. Homotopies and group isomorphisms are denoted by $\simeq$ and $\cong$ respectively. For level sets and critical sets we use the customary notation

$$
\begin{gathered}
\Phi^{c}:=\{x \in E: \Phi(x) \leq c\}, \\
K:=\{x \in E: \nabla \Phi(x)=0\} \quad \text { and } \quad K_{c}:=K \cap \Phi^{-1}(c) .
\end{gathered}
$$

Occasionally we will write $K(\Phi)$ instead of $K$ if we want to distinguish between critical sets of different functionals.

\section{Cohomology of filtered SPACES}

Let $X$ be a metric space and $A$ a closed subset of $X$. In what follows we denote the Cech cohomology of the pair $(X, A)$ with coefficients in a fixed field $\mathcal{F}$ by $H^{*}(X, A)$. It is well-known that the Cech cohomology satisfies all the EilenbergSteenrod axioms. It also has some additional properties which will be useful later.

Property 1.1. [39, Corollary 6.6.3] Let $(X, A)$ be a pair of closed subsets of a normed linear space $E, A \subset X$. Let $\Lambda$ be the family of all pairs $(U, V)$ of open subsets of $E$ such that $X \subset U$ and $A \subset V \subset U$. Then $\Lambda$ is an inverse system directed by inclusion, and

$$
H^{*}(X, A)=\underset{\Lambda}{\lim } H^{*}(U, V) .
$$

Since open sets in a normed linear space are absolute neighbourhood retracts, the Cech and the singular cohomology of $(U, V)$ coincide. It follows that for pairs $(X, A)$ as above, Property 1.1 (with singular groups on the right-hand side of (1.1)) may be taken as a definition of the Čech cohomology. See also [17, Sec. VIII.6]. Note that in [39] Property 1.1 is shown to hold for the Alexander-Spanier cohomology. However, for paracompact Hausdorff spaces the Cech and the Alexander-Spanier theories are equivalent (by [39, Corollary 6.8.8] and the five lemma applied to the exact sequence of pairs).

Property 1.2. (Strong excision) If $A$ and $B$ are closed subsets of a normed linear space $E$, then the inclusion $(A, A \cap B) \subset(A \cup B, B)$ induces an isomorphism (the so-called excision isomorphism)

$$
H^{*}(A, A \cap B) \stackrel{e x c}{\cong} H^{*}(A \cup B, B) .
$$

The above property, in a more general form, may be found in [39, Theorem 6.6.5]. See also [17, VIII.6.15]. 
In order to introduce cohomology theory of filtered spaces we will need some preliminaries. Let $\left(\mathcal{G}_{n}\right)_{n=1}^{\infty}$ be a sequence of abelian groups. We define the asymptotic group $\left[\left(\mathcal{G}_{n}\right)_{n=1}^{\infty}\right]$ by the formula

$$
\left[\left(\mathcal{G}_{n}\right)_{n=1}^{\infty}\right]:=\prod_{n=1}^{\infty} \mathcal{G}_{n} / \bigoplus_{n=1}^{\infty} \mathcal{G}_{n}
$$

In other words, in the group

$$
\prod_{n=1}^{\infty} \mathcal{G}_{n}=\left\{\left(\xi_{n}\right)_{n=1}^{\infty}: \xi_{n} \in \mathcal{G}_{n}\right\}
$$

we introduce the equivalence relation $\left(\xi_{n}\right)_{n=1}^{\infty} \sim\left(\eta_{n}\right)_{n=1}^{\infty}$ if and only if $\xi_{n}=\eta_{n}$ for almost all $n \geq 1$, and set

$$
\left[\left(\mathcal{G}_{n}\right)_{n=1}^{\infty}\right]=\prod_{n=1}^{\infty} \mathcal{G}_{n} / \sim
$$

If in particular $\mathcal{G}_{n}=\mathcal{G}$ for almost all $n$, we will write $[\mathcal{G}]$ instead of $\left[\left(\mathcal{G}_{n}\right)_{n=1}^{\infty}\right]$. Note that the above construction of asymptotic groups generalizes immediately to modules.

Assume now that $E$ is a real Hilbert space and there is a filtration $\left(E_{n}\right)_{n=1}^{\infty}$ of $E$. Suppose that a sequence $\left(d_{n}\right)_{n=1}^{\infty}$ of nonnegative integers is given and let $\mathcal{E}=\left\{E_{n}, d_{n}\right\}_{n=1}^{\infty}$. If $(X, A)$ is a closed pair of subsets of $E$, then for any integer $q$ we define the $q$-th $\mathcal{E}$-cohomology group of $(X, A)$ with coefficients in $\mathcal{F}$ by the formula

$$
H_{\mathcal{E}}^{q}(X, A):=\left[\left(H^{q+d_{n}}\left(X \cap E_{n}, A \cap E_{n}\right)\right)_{n=1}^{\infty}\right] .
$$

Since $\mathcal{F}$ is a field, $H_{\mathcal{E}}^{*}(X, A)$ is in fact a (graded) vector space over $\mathcal{F}$.

As admissible morphisms in the category of closed pairs in $E$ we take all mappings $f:(X, A) \rightarrow(Y, B)$ which preserve the filtration. It is clear that each such $f$ induces a homomorphism

$$
f^{*}: H_{\mathcal{E}}^{*}(Y, B) \rightarrow H_{\mathcal{E}}^{*}(X, A)
$$

given by the formula $f^{*}:=\left[f_{n}^{*}\right]$, or more precisely, by

$$
f^{*}\left[\left(\xi_{n}\right)_{n=1}^{\infty}\right]:=\left[\left(f_{n}^{*}\left(\xi_{n}\right)\right)_{n=1}^{\infty}\right],
$$

where $f_{n}:=\left.f\right|_{\left(X \cap E_{n}, A \cap E_{n}\right)}$ and $\xi_{n} \in H^{*+d_{n}}\left(Y \cap E_{n}, B \cap E_{n}\right)$.

The coboundary homomorphism $\delta^{*}: H^{*}(A) \rightarrow H^{*+1}(X, A)$ is defined by setting $\delta^{*}:=\left[\delta_{n}^{*}\right]$, where $\delta_{n}^{*}: H^{q+d_{n}}\left(A \cap E_{n}\right) \rightarrow H^{q+d_{n}+1}\left(X \cap E_{n}, A \cap E_{n}\right)$ is the usual coboundary homomorphism in the Cech theory.

A homotopy $G$ between two admissible mappings $f, g:(X, A) \rightarrow(Y, B)$ will be called admissible or filtration-preserving if $G\left([0,1] \times\left(X \cap E_{n}\right)\right) \subset E_{n}$ for almost all $n$. It is easy to see that $H_{\mathcal{E}}^{*}$ is a cofunctor in our (extraordinary) cohomology theory of closed pairs in $E$ and filtration-preserving mappings. More precisely, we have the following:

Proposition 1.3. (i) (Contravariance of $H_{\mathcal{E}}^{*}$ ) If id is the identity mapping on $(X, A)$, then $\mathrm{id}^{*}$ is the identity on $H_{\mathcal{E}}^{*}(X, A)$, and if $f:(X, A) \rightarrow(Y, B)$ and $g:(Y, B) \rightarrow(Z, C)$ are admissible, then $(g \circ f)^{*}=f^{*} \circ g^{*}$.

(ii) (Naturality of $\delta^{*}$ ) If $f:(X, A) \rightarrow(Y, B)$ is admissible, then $\delta^{*}\left(\left.f\right|_{A}\right)^{*}=f^{*} \delta^{*}$. 
(iii) (Exactness) For each pair $(X, A), A \subset X$, of closed subsets of $E$, let $i: A \subset$ $X$ and $j: X \subset(X, A)$ be the inclusions. Then the cohomology sequence

$$
\cdots \longrightarrow H_{\mathcal{E}}^{q}(X, A) \stackrel{j^{*}}{\longrightarrow} H_{\mathcal{E}}^{q}(X) \stackrel{i^{*}}{\longrightarrow} H_{\mathcal{E}}^{q}(A) \stackrel{\delta^{*}}{\longrightarrow} H_{\mathcal{E}}^{q+1}(X, A) \longrightarrow \cdots
$$

is exact.

(iv) (Strong excision) If $A, B$ are closed subsets of $E$, then the inclusion $(A, A \cap B) \subset(A \cup B, B)$ induces the excision isomorphism

$$
H_{\mathcal{E}}^{*}(A, A \cap B) \stackrel{e x c}{\cong} H_{\mathcal{E}}^{*}(A \cup B, B) .
$$

(v) (Homotopy invariance) If $f, g:(X, A) \rightarrow(Y, B)$ are admissible and homotopic by an admissible homotopy, then $f^{*}=g^{*}$.

(vi) (Exact sequence of a triple) For each triple $(X, A, B)$, where $B \subset A \subset X$ are closed subsets of $E$, let $i:(A, B) \subset(X, B)$ and $j:(X, B) \subset(X, A)$ be the inclusions. Then there exists a homomorphism $\delta^{*}: H_{\mathcal{E}}^{*}(A, B) \rightarrow H_{\mathcal{E}}^{*+1}(X, A)$ such that the cohomology sequence

$$
\cdots \longrightarrow H_{\mathcal{E}}^{q}(X, A) \stackrel{j^{*}}{\longrightarrow} H_{\mathcal{E}}^{q}(X, B) \stackrel{i^{*}}{\longrightarrow} H_{\mathcal{E}}^{q}(A, B) \stackrel{\delta^{*}}{\longrightarrow} H_{\mathcal{E}}^{q+1}(X, A) \longrightarrow \cdots
$$

is exact.

The proofs follow immediately from the definitions and the corresponding properties of ordinary cohomology (see [17, 39] and Property 1.2). E.g., for a fixed $n$ one has the exact sequence

$$
\begin{aligned}
\cdots & \rightarrow H^{q+d_{n}}\left(X \cap E_{n}, A \cap E_{n}\right) \stackrel{j_{n}^{*}}{\rightarrow} H^{q+d_{n}}\left(X \cap E_{n}, B \cap E_{n}\right) \\
& \stackrel{i_{n}^{*}}{\rightarrow} H^{q+d_{n}}\left(A \cap E_{n}, B \cap E_{n}\right) \stackrel{\delta_{n}^{*}}{\rightarrow} H^{q+d_{n}+1}\left(X \cap E_{n}, A \cap E_{n}\right) \rightarrow \cdots,
\end{aligned}
$$

and this gives (vi).

Note that $H_{\mathcal{E}}^{*}$ satisfies all the Eilenberg-Steenrod axioms for cohomology ((i)(v) above) except the dimension axiom which is satisfied only in the trivial case $E_{n}=E$ and $d_{n}=0$ for almost all $n$. Note also that (iii) is a special case of (vi) (take $B=\emptyset$ ).

Instead of the dimension axiom we have the following basic example:

Example 1.4. Suppose that $F$ is a closed subspace of $E, \operatorname{dim}\left(F \cap E_{n}\right)=k_{n}$ and $d:=\lim _{n \rightarrow \infty}\left(k_{n}-d_{n}\right)$ exists, $d \in \mathbf{Z} \cup\{ \pm \infty\}$. Given $p \in F$ and $r>\|p\|$, let $D:=\bar{B}(p, r) \cap F$ and $S:=S(p, r) \cap F$. For each $n \geq 1, D \cap E_{n}$ is a closed ball with boundary $S \cap E_{n}$ and $\operatorname{dim}\left(D \cap E_{n}\right)=k_{n}$. So if $d=+\infty$ or $-\infty$, then for large $n, H^{q+d_{n}}\left(D \cap E_{n}, S \cap E_{n}\right)=0$, and $H_{\mathcal{E}}^{q}(D, S)=0$ for all $q \in \mathbf{Z}$. If $d \neq \pm \infty$, then $q+d_{n}=q+k_{n}-d$ for almost all $n$ and

$$
H_{\mathcal{E}}^{q}(D, S)= \begin{cases}{[\mathcal{F}]} & \text { for } q=d \\ {[0]} & \text { otherwise }\end{cases}
$$

\section{Critical groups}

Let $E$ be a real Hilbert space and $\mathcal{E}=\left(E_{n}\right)_{n=1}^{\infty}$ a filtration of $E$. Denote the orthogonal projector of $E$ onto $E_{n}$ by $P_{n}$. Observe that if $Z \subset E$ is a compact set, then $P_{n} x \rightarrow x$ as $n \rightarrow \infty$, uniformly for $x \in Z$.

Let $\Phi \in C^{1}(E, \mathbf{R})$. A sequence $\left(y_{j}\right)_{j=1}^{\infty}$ is said to be a $(P S)^{*}$-sequence (with respect to $\mathcal{E}$ ) if

$\Phi\left(y_{j}\right)$ is bounded, $y_{j} \in E_{n_{j}}$ for some $n_{j}, n_{j} \rightarrow \infty$ and $P_{n_{j}} \nabla \Phi\left(y_{j}\right) \rightarrow 0$ as $j \rightarrow \infty$. 
If each (PS)*-sequence has a convergent subsequence, then $\Phi$ is said to satisfy the $(P S)^{*}$-condition (with respect to $\mathcal{E}$ ), and if this is true for each $(\mathrm{PS})^{*}$-sequence contained in a closed set $N$, then $\Phi$ is said to satisfy the (PS)*-condition on $N$.

The (PS)*-condition (in a slightly different form) has been introduced by Bahri and Berestycki $[4,5]$ and $\mathrm{Li}$ and Liu [25]. Note that if $\Phi$ satisfies (PS)*, then each convergent subsequence of $\left(y_{j}\right)$ tends to a critical point of $\Phi$. Moreover, $\Phi$ satisfies the usual Palais-Smale condition (PS). Indeed, suppose $\left(x_{j}\right)$ is a sequence such that $\Phi\left(x_{j}\right)$ is bounded and $\nabla \Phi\left(x_{j}\right) \rightarrow 0$. For each $j$ there exists an $n_{j} \geq j$ such that setting $y_{j}:=P_{n_{j}} x_{j}$, we obtain $\left|\Phi\left(x_{j}\right)-\Phi\left(y_{j}\right)\right| \leq 1,\left\|y_{j}-x_{j}\right\| \leq 1 / j$ and $\left\|\nabla \Phi\left(x_{j}\right)-\nabla \Phi\left(y_{j}\right)\right\| \leq 1 / j$. Hence $P_{n_{j}} \nabla \Phi\left(y_{j}\right) \rightarrow 0$, so $\left(y_{j}\right)$, and therefore also $\left(x_{j}\right)$, has a convergent subsequence.

In what follows we will usually assume that $\Phi$ satisfies (PS)* on the whole space $E$. However, let us remark that our results remain valid if (PS)* is satisfied only on a suitable closed subset of $E$.

Definition 2.1. Let $N \subset E-K$, where $K$ is the critical set of $\Phi$. A mapping $V: N \rightarrow E$ is called a gradient-like vector field for $\Phi$ on $N$ if

(i): $\mathrm{V}$ is locally Lipschitz continuous;

(ii): $\|V(x)\| \leq 1$ for all $x \in N$;

(iii): there is a function $\beta: N \rightarrow \mathbf{R}_{+}$such that $\langle\nabla \Phi(x), V(x)\rangle \geq \beta(x)$ for all $x \in N$ and $\inf _{z \in Z} \beta(z)>0$ for any set $Z \subset N$ which is bounded away from $K$ and such that $\sup _{z \in Z}|\Phi(z)|<\infty$.

We say that a gradient-like vector field $V$ for $\Phi$ on $N$ is related to $\mathcal{E}$ (or $\mathcal{E}$-related) if the mapping $\left.V\right|_{Z}$ preserves the filtration $\left(E_{n}\right)_{n=1}^{\infty}$ on any set $Z \subset N$ which is bounded away from $K$ and such that $\sup _{Z}|\Phi|<\infty$.

Lemma 2.2. Let $N$ be an open subset of $E$. If $\Phi \in C^{1}(E, \mathbf{R})$ satisfies the (PS)*condition, then there exists an $\mathcal{E}$-related gradient-like vector field $V$ for $\Phi$ on $N-K$.

Proof. Let

$$
N_{k}:=\left\{x \in N: d(x, K)>\frac{1}{k},|\Phi(x)|<k\right\} .
$$

Clearly, for each $k \geq 1$ the set $N_{k}$ is open, $N_{k} \subset N_{k+1}$ and $\bigcup_{k=1}^{\infty} N_{k}=N-K$. Hence there is an integer $k_{0} \geq 1$ such that $N_{k} \neq \emptyset$ for $k \geq k_{0}$.

For each $k \geq k_{0}$ and $n \geq 1$, let

$$
\gamma_{n}(k):=\inf \left\{\left\|P_{n} \nabla \Phi(y)\right\|: y \in N_{k} \cap E_{n}\right\}
$$

and

Evidently,

$$
\gamma(k):=\frac{1}{2} \liminf _{n \rightarrow \infty} \gamma_{n}(k)
$$

$$
\gamma(k+1) \leq \gamma(k)
$$

Moreover,

$$
\gamma(k)>0
$$

for any $k \geq k_{0}$. For if $\gamma(k)=0$, then there is a sequence $\left(y_{j}\right), y_{j} \in N_{k} \cap E_{n_{j}}$, such that $n_{j} \rightarrow \infty$ and $P_{n_{j}} \nabla \Phi\left(y_{j}\right) \rightarrow 0$ as $j \rightarrow \infty$. Since $\left|\Phi\left(y_{j}\right)\right|<k$ for any $j$, it follows from (PS)* that after passing to a subsequence, $y_{j} \rightarrow y \in \bar{N}_{k}$. Therefore $y \notin K$. But $\nabla \Phi(y)=\lim _{j \rightarrow \infty} P_{n_{j}} \nabla \Phi\left(y_{j}\right)=0$, a contradiction. 
For any $x \in N-K$, let

$$
m(x):=\min \left\{k \geq k_{0}: x \in N_{k}\right\}
$$

and define a function $\beta: N-K \rightarrow \mathbf{R}_{+}$by the formula

$$
\beta(x):=\frac{1}{2} \gamma(m(x)+1), \quad x \in N-K .
$$

If $Z \subset N-K, Z$ is bounded away from $K$ and $\sup _{Z}|\Phi|<\infty$, then there is a $k \geq k_{0}$ such that $Z \subset N_{k}$. Hence by (2.1), (2.2),

$$
\inf _{z \in Z} \beta(z) \geq \frac{1}{2} \gamma(k+1)>0 .
$$

Let $x \in N-K$ and define

$$
W_{x}:=\left\{w \in S:\langle\nabla \Phi(x), w\rangle>\frac{1}{2} \gamma(m(x))\right\},
$$

where $S:=\{w \in E:\|w\|=1\}$. Observe that $W_{x}$ is open (in $S$ ) and nonempty (because $\|\nabla \Phi(x)\|=\lim _{n \rightarrow \infty}\left\|P_{n} \nabla \Phi\left(P_{n} x\right)\right\| \geq \liminf _{n \rightarrow \infty} \gamma_{n}(m(x))=2 \gamma(m(x))$ ). Denote $S_{n}:=S \cap E_{n}$. Since $\operatorname{cl}\left(\bigcup_{n=1}^{\infty} S_{n}\right)=S$, the number

$$
n(x):=\min \left\{n \geq 1: W_{x} \cap S_{n} \neq \emptyset\right\}
$$

is well-defined. Let $w(x)$ be an arbitrary point of $W_{x} \cap S_{n(x)}$. Since $\nabla \Phi$ is continuous and $N_{m(x)}$ is open, there exists an open neighbourhood $U(x)$ of $x$ such that

$$
\begin{gathered}
U(x) \subset N_{m(x)}-N_{m(x)-2}, \\
\langle\nabla \Phi(y), w(x)\rangle>\frac{1}{2} \gamma(m(x)) \quad \forall y \in U(x)
\end{gathered}
$$

and

$$
\|\nabla \Phi(y)-\nabla \Phi(x)\|<\frac{1}{2} \gamma(m(x)) \quad \forall y \in U(x)
$$

By (2.4),

$$
m(y)=m(x) \text { or } m(y)=m(x)-1 \quad \text { whenever } y \in U(x) .
$$

The open covering $\{U(x)\}_{x \in N-K}$ admits a locally finite Lipschitz continuous partition of unity $\left\{\lambda_{j}\right\}_{j \in J}$ subordinate to it. For each $j \in J$ there is an $x_{j} \in N-K$ such that $\operatorname{supp} \lambda_{j} \subset U\left(x_{j}\right)$. Define $V: N-K \rightarrow E$ by the formula

$$
V(y):=\sum_{j \in J} \lambda_{j}(y) w\left(x_{j}\right), \quad y \in N-K
$$

Clearly, $V$ is locally Lipschitz continuous and $\|V(y)\| \leq 1$ for $y \in N-K$.

Let $y \in N-K$. If $\lambda_{j}(y) \neq 0$, then $y \in U\left(x_{j}\right)$, and in view of $(2.7), m\left(x_{j}\right) \leq$ $m(y)+1$. Therefore, by $(2.5)$ and (2.1),

$$
\langle\nabla \Phi(y), V(y)\rangle=\sum_{j \in J} \lambda_{j}(y)\left\langle\nabla \Phi(y), w\left(x_{j}\right)\right\rangle>\frac{1}{2} \gamma(m(y)+1)=\beta(y) .
$$

This together with (2.3) shows that $V$ is a gradient-like vector field for $\Phi$ on $N-K$.

It remains to show that $V$ is related to $\mathcal{E}$. Let $Z \subset N-K$ be bounded away from $K$ and such that $\sup _{Z}|\Phi|<\infty$. Then $Z \subset N_{m}$ for some $m \geq k_{0}$. There exists an $n_{0}$ such that $\gamma_{n}(k)>\gamma(k)$ for all $n \geq n_{0}$ and $k_{0} \leq k \leq m$. It follows that

$$
\left\|P_{n} \nabla \Phi(y)\right\|>\gamma(k) \quad \text { whenever } n \geq n_{0}, k_{0} \leq k \leq m \text { and } y \in N_{k} \cap E_{n} .
$$


Let $n \geq n_{0}$ and $y \in Z \cap E_{n}$. If $\lambda_{j}(y) \neq 0$, then $y \in U\left(x_{j}\right)$, and by $(2.7), m(y) \leq$ $m\left(x_{j}\right)$. Moreover, $m(y) \leq m$ since $Z \subset N_{m}$. Let $\bar{w}:=\left\|P_{n} \nabla \Phi(y)\right\|^{-1} P_{n} \nabla \Phi(y) \in$ $S_{n}$. By (2.8) (with $\left.k=m(y)\right)$ and (2.6),

$$
\begin{aligned}
\left\langle\nabla \Phi\left(x_{j}\right), \bar{w}\right\rangle & =\langle\nabla \Phi(y), \bar{w}\rangle-\left\langle\nabla \Phi(y)-\nabla \Phi\left(x_{j}\right), \bar{w}\right\rangle \\
& =\left\|P_{n} \nabla \Phi(y)\right\|-\left\langle\nabla \Phi(y)-\nabla \Phi\left(x_{j}\right), \bar{w}\right\rangle \\
& >\gamma(m(y))-\frac{1}{2} \gamma\left(m\left(x_{j}\right)\right) \geq \frac{1}{2} \gamma\left(m\left(x_{j}\right)\right) .
\end{aligned}
$$

Hence $\bar{w} \in W_{x_{j}} \cap S_{n}$. It follows that $W_{x_{j}} \cap S_{n} \neq \emptyset$, and by the definition of $n(x)$, $n\left(x_{j}\right) \leq n$. Therefore $w\left(x_{j}\right) \in E_{n\left(x_{j}\right)} \subset E_{n}$ and $V(y) \in E_{n}$.

Definition 2.3. Let $A$ be an isolated compact subset of the critical set $K$ of a functional $\Phi \in C^{1}(E, \mathbf{R})$. A pair $\left(W, W^{-}\right)$of closed subsets of $E$ is said to be an admissible pair for $\Phi$ and $A$ with respect to $\mathcal{E}$ if the following conditions are satisfied:

(i): $W$ is bounded away from $K-A, W^{-} \subset \partial W$ and $A \subset \operatorname{int}(W)$;

(ii): $\left.\Phi\right|_{W}$ is bounded;

(iii): there are a neighbourhood $N$ of $W$ and an $\mathcal{E}$-related gradient-like vector field $V$ for $\Phi$ on $N-A$;

(iv): $W^{-}$is the union of finitely many (possibly intersecting) closed sets each of which lies on a $C^{1}$-manifold of codimension $1, V$ is transversal to each of these manifolds at points of $W^{-}$, the flow $\eta$ of $-V$ can leave $W$ only via $W^{-}$, and if $x \in W^{-}$, then $\eta(t, x) \notin W$ for any $t>0$.

The gradient-like vector field $V$ corresponding to $\left(W, W^{-}\right)$will be called an admissible field. In what follows we will usually omit the expressions "related to $\mathcal{E}$ " and "with respect to $\mathcal{E}$ ".

Remark 2.4. Assume that a pair $\left(W, W^{-}\right)$of closed sets satisfies the conditions of Definition 2.3 except that the gradient-like field $V$ in (iii) is defined only on a neighbourhood $N$ of $\partial W$. If $\Phi$ satisfies (PS)*, then, using Lemma 2.2 and partition of unity, it is easy to construct a gradient-like field $\widetilde{V}: \widetilde{N}-A \rightarrow E$, where $\widetilde{N}=$ $N \cup W$. Thus $\left(W, W^{-}\right)$is an admissible pair for $\Phi$ and $A$.

Proposition 2.5. Assume that $\Phi \in C^{1}(E, \mathbf{R})$ satisfies $(P S)^{*}$. Let $a<b, W:=$ $\Phi^{-1}([a, b])$ and $W^{-}:=\Phi^{-1}(a)$. If $A:=K \cap \operatorname{int}(W)$ and $W$ is bounded away from $K-A$, then the pair $\left(W, W^{-}\right)$is admissible for $\Phi$ and $A$.

Proof. Clearly, there exists an open neighbourhood $N$ of $W$ such that $N$ is bounded away from $K-A$. In view of Lemma 2.2 , there is an $\mathcal{E}$-related gradient-like vector field $V$ for $\Phi$ on $N-A$. Since $\langle\nabla \Phi(x), V(x)\rangle>0$ whenever $x \in W^{-},\left(W, W^{-}\right)$is an admissible pair.

Recall that $S(p, \delta)=\{x \in E:\|x-p\|=\delta\}$.

Proposition 2.6. Suppose that $\Phi \in C^{1}(E, \mathbf{R})$ satisfies $(P S)^{*}$ and has an isolated critical point $p$. For each open neighbourhood $U$ of $p$ there exists an admissible pair $\left(W, W^{-}\right)$for $\Phi$ and $p$ such that $W \subset U$ and $\left.\Phi\right|_{W^{-}}<c:=\Phi(p)$. Moreover, there is a $\delta_{1}>0$ such that $\bar{B}\left(p, \delta_{1}\right) \subset \operatorname{int}(W)$ and if $x \in S\left(p, \delta_{1}\right) \cap \Phi^{c}$, then $\eta(t, x) \in W^{-}$ for some $t>0$ ( $\eta$ is the flow of $-V$ ). 
Proof. Choose $\delta>0$ such that $0<\delta<d(p, K-\{p\}), \bar{B}(p, \delta) \subset U$ and $\sup _{B(p, \delta)}|\Phi|$ $<\infty$. Let $V: \bar{B}(p, \delta)-\{p\} \rightarrow E$ be a gradient-like vector field related to $\mathcal{E}$ and let

$$
\alpha:=\inf \left\{\beta(x): \frac{\delta}{2} \leq\|x-p\| \leq \delta\right\}
$$

$(\beta($.$) is the function in (iii) of Definition 2.1). Choose \varepsilon>0$ with

$$
0<\varepsilon<\frac{\alpha \delta}{4} .
$$

Let $\delta_{1}, \delta_{2}>0$ be such that $\delta_{2}<\delta_{1} / 2<\delta / 4$ and

$$
\bar{B}\left(p, \delta_{1}\right) \subset\{x \in E:|\Phi(x)-c|<\varepsilon\} .
$$

Set $N:=\bar{B}(p, \delta)$. Define now a locally Lipschitz continuous function $\omega: N \rightarrow$ $[0,1]$ such that $\omega(x)=0$ in a neighbourhood of $p, \omega(x)=1$ for $\delta_{2} \leq\|x-p\| \leq \delta$, and consider the initial value problem

Having all this, define

$$
\frac{d \sigma}{d t}=-\omega(\sigma) V(\sigma), \quad \sigma(0, x)=x \in N .
$$

$$
W:=\left\{\sigma(t, x): t \geq 0, x \in \bar{B}\left(p, \delta_{1}\right), \Phi(\sigma(t, x)) \geq c-\varepsilon\right\}
$$

and

$$
W^{-}:=W \cap \Phi^{-1}(c-\varepsilon) .
$$

We will show that $W \subset B(p, \delta)$. Assuming the contrary, there are $x \in \bar{B}\left(p, \delta_{1}\right)$ and $0 \leq t_{1}<t_{2}$ such that $\delta / 2<\|\sigma(t, x)-p\|<\delta$ for $t \in\left(t_{1}, t_{2}\right)$ and $\left\|\sigma\left(t_{1}, x\right)-p\right\|=$ $\delta / 2,\left\|\sigma\left(t_{2}, x\right)-p\right\|=\delta$. Hence

$$
\frac{\delta}{2} \leq\left\|\sigma\left(t_{2}, x\right)-\sigma\left(t_{1}, x\right)\right\| \leq \int_{t_{1}}^{t_{2}}\|V(\sigma(s, x))\| d s \leq t_{2}-t_{1} .
$$

Moreover, by Definition 2.1,

$$
\begin{aligned}
& \Phi\left(\sigma\left(t_{2}, x\right)\right)-\Phi\left(\sigma\left(t_{1}, x\right)\right)=\int_{t_{1}}^{t_{2}} \frac{d}{d s} \Phi(\sigma(s, x)) d s \\
& =\int_{t_{1}}^{t_{2}}\langle\nabla \Phi(\sigma(s, x)),-V(\sigma(s, x))\rangle d s \leq-\alpha\left(t_{2}-t_{1}\right) .
\end{aligned}
$$

Therefore, in view of (2.9), (2.10),

$$
\Phi\left(\sigma\left(t_{2}, x\right)\right) \leq \Phi\left(\sigma\left(t_{1}, x\right)\right)-\alpha\left(t_{2}-t_{1}\right) \leq c+\varepsilon-\frac{\alpha \delta}{2}<c-\varepsilon,
$$

a contradiction.

The set $W$ is closed. For if $y_{n}:=\sigma\left(t_{n}, x_{n}\right) \in W$, where $x_{n} \in \bar{B}\left(p, \delta_{1}\right), t_{n} \geq 0$ and $y_{n} \rightarrow y \in \partial W$, then $x_{n}$ may be chosen so that $\sigma\left(t, x_{n}\right) \notin B\left(p, \delta_{2}\right)$ for $0 \leq$ $t \leq t_{n}$. Since $\Phi\left(\sigma\left(t_{n}, x_{n}\right)\right) \geq c-\varepsilon$, we obtain that the sequence $\left(t_{n}\right)$ is bounded. Hence $t_{n} \rightarrow t \geq 0$ (possibly after passing to a subsequence), $x_{n}=\sigma\left(-t_{n}, y_{n}\right) \rightarrow$ $\sigma(-t, y)=: x \in \bar{B}\left(p, \delta_{1}\right)$, and $y=\sigma(t, x) \in W$.

The set $W^{-}$is obviously closed, and it is a subset of the submanifold $B(p, \delta) \cap$ $\Phi^{-1}(c-\varepsilon)$. Since the mapping $t \mapsto \sigma(t, x)$ is transversal to this manifold, $\left(W, W^{-}\right)$ is an admissible pair (and $\left.\left.\Phi\right|_{W^{-}}<c\right)$.

To prove the second conclusion, note that since $\inf \left\{\beta(x): \delta_{1} / 2 \leq\|x-p\| \leq \delta\right\}$ $>0$, there exists an $\varepsilon_{0}>0$ such that if $x \in S\left(p, \delta_{1}\right)$ and $\Phi(x) \leq c$, then $\Phi(\sigma(t, x))$ $\leq c-\varepsilon_{0}$ whenever $\|\sigma(t, x)\|=\delta_{1} / 2$. Choosing $\delta_{2}$ smaller if necessary, we obtain 
$\Phi(x)>c-\varepsilon_{0}$ for each $x \in B\left(p, \delta_{2}\right)$. Therefore $\sigma(t, x)$ cannot enter $B\left(p, \delta_{2}\right)$. Since $\omega(x)=1$ and $\beta(x)$ is bounded away from 0 as $\delta_{2} \leq\|x-p\| \leq \delta, \Phi\left(\sigma\left(t_{0}, x\right)\right)=c-\varepsilon$ for some $t_{0}$, and $\eta\left(t_{0}, x\right)=\sigma\left(t_{0}, x\right) \in W^{-}$.

From now on we assume that the sequence $\left(d_{n}\right)$ has been given and that $\mathcal{E}=$ $\left\{E_{n}, d_{n}\right\}$. Let $p$ be an isolated critical point of a functional $\Phi \in C^{1}(E, \mathbf{R})$ satisfying the (PS)*-condition and let $\left(W, W^{-}\right)$be an admissible pair for $\Phi$ and $p$. We define the $q$-th critical group $(q \in \mathbf{Z})$ of $\Phi$ at $p$ with respect to $\mathcal{E}$ by the formula

$$
c_{\mathcal{E}}^{q}(\Phi, p):=H_{\mathcal{E}}^{q}\left(W, W^{-}\right)
$$

Proposition 2.6 asserts the existence of an admissible pair $\left(W, W^{-}\right)$. We will prove now that $c_{\mathcal{E}}^{q}(\Phi, p), q \in \mathbf{Z}$, is well-defined, i.e., it does not depend on the particular choice of such a pair.

Proposition 2.7. Suppose that $\Phi$ satisfies $(P S)^{*}$ and $\left(W_{1}, W_{1}^{-}\right),\left(W_{2}, W_{2}^{-}\right)$are two admissible pairs for $\Phi$ and an isolated critical point $p$. Then $H_{\mathcal{E}}^{*}\left(W_{1}, W_{1}^{-}\right) \cong$ $H_{\mathcal{E}}^{*}\left(W_{2}, W_{2}^{-}\right)$.

Proof. To $\left(W_{i}, W_{i}^{-}\right)$there correspond a neighbourhood $N_{i}$ of $W_{i}$ and an admissible vector field $V_{i}$ on $N_{i}-\{p\}, i=1,2$. According to Proposition 2.6, there is an admissible pair $\left(W, W^{-}\right)$for $\Phi$ and $p$ such that $W \subset \operatorname{int}\left(W_{1}\right) \cap \operatorname{int}\left(W_{2}\right)$. It suffices to show that

$$
H_{\mathcal{E}}^{*}\left(W_{1}, W_{1}^{-}\right) \cong H_{\mathcal{E}}^{*}\left(W, W^{-}\right) .
$$

Assuming that $\left(W, W^{-}\right)$is constructed as in Proposition 2.6, we easily obtain (using an appropriate partition of unity) a gradient-like vector field $\widetilde{V}$ which is admissible for both $\left(W, W^{-}\right)$and $\left(W_{1}, W_{1}^{-}\right)$. Note in particular that since $W^{-} \subset$ $\Phi^{-1}(c-\varepsilon)$, where $c:=\Phi(p)$, the flow $\widetilde{\eta}$ of $-\widetilde{V}$ cannot re-enter $W$ after leaving it.

Consider the initial value problem

$$
\frac{d \sigma}{d t}=-\widetilde{\omega}(\sigma) \widetilde{V}(\sigma), \quad \sigma(0, x)=x \in W_{1},
$$

where $\widetilde{\omega}: N_{1} \rightarrow[0,1]$ is a locally Lipschitz continuous function such that $\widetilde{\omega} \equiv 0$ on $\bar{B}\left(p, \delta_{0} / 2\right), \widetilde{\omega} \equiv 1$ on $N_{1}-B\left(p, \delta_{0}\right)$, and $\delta_{0}$ is chosen so that $\bar{B}\left(p, \delta_{0}\right) \subset \operatorname{int}(W)$. Observe that whenever $n$ is large enough, then

$$
\sigma(t, x) \in W_{1} \cap E_{n} \text { provided } x \in W_{1} \cap E_{n} \text { and } \sigma(t, x) \in W_{1}
$$

(because the mapping $W_{1} \ni x \mapsto \widetilde{\omega}(x) \widetilde{V}(x)$ preserves the filtration).

Since the mapping $W_{1} \ni x \mapsto \widetilde{\omega}(x) \widetilde{V}(x)$ is locally Lipschitz continuous and bounded, it follows that for a given $x \in W_{1}$ either there is a unique $t=t(x) \in[0, \infty)$ such that $\sigma(t(x), x) \in W_{1}^{-}$or $\sigma(t, x) \in W_{1}$ for all $t \geq 0$. In the latter case we set $t(x)=+\infty$. The implicit function theorem and the transversality condition on $W_{1}^{-}$ imply that the function $x \mapsto t(x)$ is continuous on the set $\left\{x \in W_{1}: t(x)<\infty\right\}$.

Let $A:=\left\{\sigma(t, x): t \geq 0, x \in W^{-}\right\} \cap W_{1}$ and let $\widetilde{W}:=W \cup A, \widetilde{W}^{-}:=\widetilde{W} \cap W_{1}^{-}$. Then $\left(\widetilde{W}, \widetilde{W}^{-}\right)$is an admissible pair for $\Phi$ and $p$, and $\widetilde{V}$ is an admissible field. Since

$$
\begin{aligned}
\Phi(\sigma(t, x))-\Phi(x) & =\int_{0}^{t} \widetilde{\omega}(\sigma(s, x))\langle\nabla \Phi(\sigma(s, x)),-\widetilde{V}(\sigma(s, x))\rangle d s \\
& \leq-\int_{0}^{t} \widetilde{\omega}(\sigma(s, x)) \widetilde{\beta}(\sigma(s, x)) d s,
\end{aligned}
$$


where $\widetilde{\beta}$ corresponds to $\widetilde{V}$ (in the sense of (iii) of Definition 2.1 ), and since $\widetilde{\omega}=1$ and $\widetilde{\beta}$ is bounded away from 0 on $W_{1}-\operatorname{int}(W)$, it follows that $t(x)<\infty$ whenever $x \in A$. Therefore the mapping

$$
[0,1] \times A \ni(\lambda, x) \mapsto \sigma(\lambda t(x), x) \in A
$$

is a strong deformation retraction of $A$ onto $\widetilde{W}^{-}$. Since it preserves the filtration (cf. $(2.11))$, we have $H_{\mathcal{E}}^{*}\left(A, \widetilde{W}^{-}\right)=0$. Now the exactness of the cohomology sequence of the triple $\left(\widetilde{W}, A, \widetilde{W}^{-}\right)$and the excision property imply that

$$
H_{\mathcal{E}}^{*}\left(\widetilde{W}, \widetilde{W}^{-}\right) \cong H_{\mathcal{E}}^{*}(\widetilde{W}, A) \stackrel{e x c}{\cong} H_{\mathcal{E}}^{*}\left(W, W^{-}\right)
$$

Let $W_{0}=\widetilde{W} \cup W_{1}^{-}$. Then $\left(W_{0}, W_{1}^{-}\right)$is an admissible pair for $\Phi$ and $p$. Excising again, we see that

$$
H_{\mathcal{E}}^{*}\left(\widetilde{W}, \widetilde{W}^{-}\right) \stackrel{e x c}{\cong} H_{\mathcal{E}}^{*}\left(W_{0}, W_{1}^{-}\right)
$$

We will show that $H_{\mathcal{E}}^{*}\left(W_{0}, W_{1}^{-}\right) \cong H_{\mathcal{E}}^{*}\left(W_{1}, W_{1}^{-}\right)$. There is a $T>0$ such that for any $x \in W_{1}$ either $\sigma(t, x) \in \widetilde{W}$ for $T \leq t \leq t(x)$ or $T \geq t(x)$. Indeed, if $\sigma(t, x) \notin \widetilde{W}$, then by (2.12), $\Phi(\sigma(t, x)) \leq \Phi(x)-t \beta$, where $\beta:=\inf _{W_{1}-\widetilde{W}} \widetilde{\beta}(x)>0$. So we can choose $T=\left(\sup _{W_{1}} \Phi-\inf _{W_{1}} \Phi\right) / \beta$.

Consider the mapping $\xi:[0, T] \times W_{1} \rightarrow W_{1}$ given by the formula

$$
\xi(t, x):= \begin{cases}\sigma(t, x) & \text { if } 0 \leq t<t(x) \\ \sigma(t(x), x) & \text { if } t(x) \leq t \leq T\end{cases}
$$

Since the function $x \mapsto t(x)$ is continuous on the set $\left\{x \in W_{1}: t(x)<\infty\right\}$, we get that $\xi$ is a filtration-preserving deformation of the pair $\left(W_{1}, W_{1}^{-}\right)$into $\left(W_{0}, W_{1}^{-}\right)$ and $\xi\left([0, T] \times W_{0}\right) \subset W_{0}, \xi\left([0, T] \times W_{1}^{-}\right) \subset W_{1}^{-}$. It follows that the pairs $\left(W_{0}, W_{1}^{-}\right)$ and $\left(W_{1}, W_{1}^{-}\right)$are homotopy equivalent by filtration-preserving homotopies. Indeed, if $i:\left(W_{0}, W_{1}^{-}\right) \rightarrow\left(W_{1}, W_{1}^{-}\right)$is the inclusion and $\xi_{T}:=\xi(T,$.$) , then \xi_{T} \circ i \simeq$ id on $\left(W_{0}, W_{1}^{-}\right)$and $i \circ \xi_{T} \simeq$ id on $\left(W_{1}, W_{1}^{-}\right)$. Hence $H_{\mathcal{E}}^{*}\left(W_{0}, W_{1}^{-}\right) \cong H_{\mathcal{E}}^{*}\left(W_{1}, W_{1}^{-}\right)$, which together with (2.13), (2.14) completes the proof.

The critical groups $c_{\mathcal{E}}^{*}(\Phi, p)$ have a certain continuity property which will be useful further on.

Proposition 2.8. Suppose that $\Phi$ satisfies $(P S)^{*}, p$ is an isolated critical point of $\Phi$ and $\left(W, W^{-}\right)$is an admissible pair for $\Phi$ and $p$. There exists an $\varepsilon>0$ such that if $\widetilde{\Phi} \in C^{1}(E, \mathbf{R})$ satisfies $(P S)^{*}, \sup _{W}|\widetilde{\Phi}|<\infty, \sup _{W}\|\nabla \Phi(x)-\nabla \widetilde{\Phi}(x)\|<\varepsilon, \widetilde{\Phi}$ has only one critical point $\widetilde{p}$ in $W$ and $W$ is bounded away from $K(\widetilde{\Phi})-\{\widetilde{p}\}$, then $\left(W, W^{-}\right)$is an admissible pair for $\widetilde{\Phi}$ and $\widetilde{p}$.

Proof. Choose a neighbourhood $N$ of $W$ such that $N$ is bounded away from $K(\Phi)-$ $\{p\}$ and $\sup _{N}|\Phi|<\infty$. Let $V: N-\{p\} \rightarrow E$ be an admissible vector field for $\Phi$ and let $\bar{B}(p, \delta) \subset \operatorname{int}(W)$. Since the set $N-B(p, \delta)$ is bounded away from $K(\Phi)$, $\beta:=\inf \{\beta(x): x \in N-B(p, \delta)\}$ is positive (again, $\beta(\cdot)$ is the function in (iii) of Definition 2.1). Let $\varepsilon \in(0, \beta)$ be fixed. If $\widetilde{\Phi}$ satisfies our hypotheses, we may assume after shrinking $N$ if necessary that $\sup _{N}|\widetilde{\Phi}|<\infty$ and $\sup _{N}\|\nabla \Phi(x)-\nabla \widetilde{\Phi}(x)\|<\varepsilon$. Evidently, for $x \in N-B(p, \delta)$,

$\langle\nabla \widetilde{\Phi}(x), V(x)\rangle=\langle\nabla \Phi(x), V(x)\rangle+\langle\nabla \widetilde{\Phi}(x)-\nabla \Phi(x), V(x)\rangle \geq \beta(x)-\varepsilon \geq \beta-\varepsilon>0$. 
So $\widetilde{p} \in B(p, \delta)$ and $N-B(p, \delta)$ is bounded away from $K(\widetilde{\Phi})$. Invoking Remark 2.4, we easily conclude the proof.

Corollary 2.9. Let $\left\{\Phi_{\lambda}\right\}_{\lambda \in[0,1]}$ be a family of $C^{1}$-functionals satisfying $(P S)^{*}$, and suppose there is an open set $U$ such that each $\Phi_{\lambda}$ has a unique critical point $p_{\lambda} \in U$, $\sup \left\{\left|\Phi_{\lambda}(x)\right|: x \in U, \lambda \in[0,1]\right\}<\infty$ and the mapping $\lambda \mapsto \nabla \Phi_{\lambda}$ is continuous, uniformly in $x \in U$ (i.e., $\sup _{U}\left\|\nabla \Phi_{\mu}(x)-\nabla \Phi_{\lambda}(x)\right\| \rightarrow 0$ as $\mu \rightarrow \lambda$ ). Then $c_{\mathcal{E}}^{*}\left(\Phi_{\lambda}, p_{\lambda}\right)$ is independent of $\lambda \in[0,1]$.

Proof. Take any $\lambda \in[0,1]$ and a ball $B_{\lambda} \subset U$ around $p_{\lambda}$. There is an admissible pair $\left(W_{\lambda}, W_{\lambda}^{-}\right), W_{\lambda} \subset B_{\lambda}$, for $\Phi_{\lambda}$ and $p_{\lambda}$. By Proposition 2.8 , there is an $\varepsilon>0$ such that for each $\mu \in[0,1],|\mu-\lambda|<\varepsilon,\left(W_{\lambda}, W_{\lambda}^{-}\right)$is an admissible pair for $\Phi_{\mu}$ and $p_{\mu}$ (that $p_{\mu} \in B\left(p_{\lambda}, \delta\right) \subset W_{\lambda}$ is seen in the same way as in the proof of Proposition 2.8). Hence $c_{\mathcal{E}}^{*}\left(\Phi_{\mu}, p_{\mu}\right)=c_{\mathcal{E}}^{*}\left(\Phi_{\lambda}, p_{\lambda}\right)$. The conclusion follows from the connectedness of $[0,1]$.

Recall our earlier observation that the results above remain valid if (PS)* is satisfied on a suitable closed subset of $E$. In particular, in Lemma $2.2(\mathrm{PS})^{*}$ is needed on $\bar{N}$, in Proposition 2.6 on a closed neighbourhood of $p$, in Proposition 2.7 on a closed neighbourhood of $W_{1} \cup W_{2}$, and in Corollary 2.9 all $\Phi_{\lambda}$ should satisfy $(\mathrm{PS})^{*}$ on $\bar{U}$.

Corollary 2.10. Suppose $p$ is an isolated critical point of $\Phi$ and let $\Phi_{p}(x):=$ $\Phi(x+p)$. If there exists a closed neighbourhood $N$ of $p$ such that $\Phi$ satisfies $(P S)^{*}$ on $N$ and $\nabla \Phi$ is uniformly continuous on $N$, then $c_{\mathcal{E}}^{*}(\Phi, p)=c_{\mathcal{E}}^{*}\left(\Phi_{p}, 0\right)$.

Proof. Let $p_{n}:=P_{n} p$. We show first that $\Phi_{p-p_{n}}$ satisfies $(\mathrm{PS})^{*}$ on $\bar{B}(p, \delta)$ for $n$ large and $\delta$ small enough. Suppose $x_{k} \in \bar{B}(p, \delta) \cap E_{n_{k}}, n_{k} \rightarrow \infty$ and $P_{n_{k}} \nabla \Phi_{p-p_{n}}\left(x_{k}\right) \rightarrow 0$. Since $\nabla \Phi$ is uniformly continuous on $N,\left\|\nabla \Phi\left(x_{k}+p-p_{n}\right)-\nabla \Phi\left(x_{k}+p_{n_{k}}-p_{n}\right)\right\| \rightarrow 0$, and therefore $P_{n_{k}} \nabla \Phi\left(x_{k}+p_{n_{k}}-p_{n}\right) \rightarrow 0$ as $k \rightarrow \infty$. Moreover, $x_{k}+p_{n_{k}}-p_{n} \in N \cap$ $E_{n_{k}}$ for almost all $k$. Hence $\left(x_{k}\right)$ has a convergent subsequence. Now let $\left(W, W^{-}\right)$, $W \subset B(p, \delta)$, be an admissible pair for $\Phi$ and $p$. Choosing a larger $n$ if necessary, it follows from Proposition 2.8 that $\left(W, W^{-}\right)$is also an admissible pair for $\Phi_{p-p_{n}}$ and $p_{n}$. Since the mapping $x \mapsto x-p_{n}$ is a filtration-preserving homeomorphism and $\left(W-p_{n}, W^{-}-p_{n}\right)$ (where $W-p_{n}:=\left\{x-p_{n}: x \in W\right\}$ ) is an admissible pair for $\Phi_{p}$ and $0, c_{\mathcal{E}}^{*}(\Phi, p)=H_{\mathcal{E}}^{*}\left(W, W^{-}\right) \cong H_{\mathcal{E}}^{*}\left(W-p_{n}, W^{-}-p_{n}\right)=c_{\mathcal{E}}^{*}\left(\Phi_{p}, 0\right)$.

Remark 2.11. It is easy to see that if $A=K_{c}$, where $c$ is an isolated critical value, then Propositions 2.6 and 2.7 remain valid for $\Phi$ and $K_{c}$.

Suppose now that the critical set $K=K(\Phi)$ is compact. A pair $\left(W, W^{-}\right)$of closed subsets of $E$ will be called a globally admissible pair for $\Phi$ and $K$ with respect to $\mathcal{E}$ if $\left(W, W^{-}\right)$satisfies the conditions of Definition 2.3 with $A=K$ and $N=E$ (i.e., $K \subset \operatorname{int}(W)$ and the gradient-like vector field $V$ is defined on $E-K)$. The field $V$ will be referred to as globally admissible. We also define the critical groups of the pair $(\Phi, K)$ by setting

$$
c_{\mathcal{E}}^{q}(\Phi, K):=H_{\mathcal{E}}^{q}\left(W, W^{-}\right),
$$

where $\left(W, W^{-}\right)$is a globally admissible pair.

Proposition 2.12. Suppose that $\Phi$ satisfies $(P S)^{*}$ and the critical set $K$ is compact. Then the critical groups $c_{\mathcal{E}}^{q}(\Phi, K)$ are well-defined. 
Proof. Let $\left(W, W^{-}\right):=\left(\Phi^{-1}([a, b]), \Phi^{-1}(a)\right)$, where $a$ and $b$ are chosen in such a way that $K \subset \operatorname{int}(W)$, and let $V: E-K \rightarrow E$ be a gradient-like vector field related to $\mathcal{E}$. It is easily seen (cf. Proposition 2.5$)$ that $\left(W, W^{-}\right)$is globally admissible.

Let $\left(W_{1}, W_{1}^{-}\right)$be another globally admissible pair with a corresponding globally admissible field $\widetilde{V}$. Choose $a$ and $b$ above so that $W_{1} \subset W$. Since $\widetilde{V}$ is also globally admissible for $\left(W, W^{-}\right)$, the argument of Proposition 2.7 shows that $H_{\mathcal{E}}^{*}\left(W, W^{-}\right) \cong$ $H_{\mathcal{E}}^{*}\left(W_{1}, W_{1}^{-}\right)$(the cutoff function $\widetilde{\omega}$ should be 0 in a neighbourhood of $K$ and 1 outside a neighhbourhood $U$ of $K$, where $\left.\bar{U} \subset \operatorname{int}\left(W_{1}\right)\right)$.

Lemma 2.13. Suppose that $\Phi$ satisfies $(P S)^{*}$ and the critical set $K$ is compact. Then there exists a bounded globally admissible pair $\left(W, W^{-}\right)$for $\Phi$ and $K$.

Proof. Choose $a$ and $b$ so that $a<\Phi(x)<b$ for $x \in K$. Let $V: E-K \rightarrow E$ be a gradient-like vector field related to $\mathcal{E}$ and let $N, U$ be two neighbourhoods of $K$ such that $N$ is closed, $U$ open and $N \subset U \subset \Phi^{-1}([a, b])$. Consider the initial value problem

$$
\frac{d \sigma}{d t}=-\omega(\sigma) V(\sigma), \quad \sigma(0, x)=x,
$$

where $\omega: E \rightarrow[0,1]$ is locally Lipschitz continuous and $\omega(x)=0$ for $x \in N$, $\omega(x)=1$ for $x \notin U$. Define

$$
W:=\{\sigma(t, x): t \geq 0, x \in \bar{U}, \Phi(\sigma(t, x)) \geq a\}
$$

and

$$
W^{-}:=W \cap \Phi^{-1}(a) .
$$

It is easy to see using the argument of Proposition 2.6 that $\left(W, W^{-}\right)$is a globally admissible pair and $W, W^{-}$are bounded sets.

The critical groups $c_{\mathcal{E}}^{*}(\Phi, K)$ have a continuity property similar to the one known from the Conley index theory $[6,15]$ :

Proposition 2.14. Let $\left\{\Phi_{\lambda}\right\}_{\lambda \in[0,1]}$ be a family of $C^{1}$-functionals satisfying (PS)*. Suppose that the mapping $\lambda \mapsto \nabla \Phi_{\lambda}$ is continuous, uniformly on bounded subsets of $E$, and there exist a bounded set $N$ and a constant $C$ such that $K\left(\Phi_{\lambda}\right) \subset N$ and $\sup _{N}\left|\Phi_{\lambda}\right| \leq C$ for all $\lambda \in[0,1]$. Then $c_{\mathcal{E}}^{*}\left(\Phi_{\lambda}, K\left(\Phi_{\lambda}\right)\right)$ is independent of $\lambda$.

Proof. Choose $\lambda \in[0,1]$ and a bounded globally admissible pair $\left(W_{\lambda}, W_{\lambda}^{-}\right)$for $\Phi_{\lambda}$ and $K\left(\Phi_{\lambda}\right)$. It is easily seen from the proof of Proposition 2.8 that $\left(W_{\lambda}, W_{\lambda}^{-}\right)$is a globally admissible pair for $\Phi_{\mu}$ and $K\left(\Phi_{\mu}\right)$ whenever $|\lambda-\mu|$ is small enough. Note in particular that $\sup _{W_{\lambda}}\left|\Phi_{\mu}\right|<\infty$ (because $W_{\lambda}$ is bounded and $\sup _{W_{\lambda}}\left|\Phi_{\lambda}\right|<\infty$ ) and $K\left(\Phi_{\mu}\right) \subset W_{\lambda}$ (because $K\left(\Phi_{\mu}\right) \subset N$ and

$$
\left\|\nabla \Phi_{\mu}(x)\right\| \geq\left\|\nabla \Phi_{\lambda}(x)\right\|-\left\|\nabla \Phi_{\mu}(x)-\nabla \Phi_{\lambda}(x)\right\|>0
$$

for $x$ in a neighbourhood of $\left.N-\operatorname{int}\left(W_{\lambda}\right)\right)$. Also, using partition of unity it is easy to construct a gradient-like vector field $V_{\mu}: E-K\left(\Phi_{\mu}\right) \rightarrow E$ for $\Phi_{\mu}$ such that $V_{\mu}=V_{\lambda}$ in a neighbourhood of $\partial W_{\lambda}$. So the conclusion follows from the connectedness of $[0,1]$.

Remark 2.15. Let $M_{0}$ be a $C^{2}$ Riemannian manifold and $\left(F_{n}\right)_{n=1}^{\infty}$ a filtration of a Hilbert space $F$. Denote the orthogonal projector of $F$ onto $F_{n}$ by $Q_{n}$ and define $M:=M_{0} \times F, M_{n}:=M_{0} \times F_{n}$ and $P_{n}(x, y):=\left(x, Q_{n} y\right)$ for $(x, y) \in M_{0} \times F$. It is easy to see by inspection that the results of this and the preceding section remain valid for $M$. 


\section{Morse inequalities}

Denote $[\mathbf{Z}]:=\prod_{n=1}^{\infty} \mathbf{Z} / \bigoplus_{n=1}^{\infty} \mathbf{Z}$ and $\left[\mathbf{Z}_{+}\right]:=\left\{\left[\left(\xi_{n}\right)_{n=1}^{\infty}\right] \in[\mathbf{Z}]: \xi_{n} \geq 0\right.$ for almost all $n\}$. Let $(X, B)$ be a pair of closed subsets of $E$ with the property that for each $q \in \mathbf{Z}$ there is an $n(q)$ such that

$$
\operatorname{dim} H^{q+d_{n}}\left(X \cap E_{n}, B \cap E_{n}\right)<\infty
$$

whenever $n \geq n(q)$. Then

$$
\operatorname{dim}_{\mathcal{E}} H_{\mathcal{E}}^{q}(X, B):=\left[\left(\operatorname{dim} H^{q+d_{n}}\left(X \cap E_{n}, B \cap E_{n}\right)\right)_{n=1}^{\infty}\right]
$$

is a well-defined element of $\left[\mathbf{Z}_{+}\right]$. The sequence on the right-hand side of (3.1) will often be constant for almost all $n$. In such a case we will write $\operatorname{dim}_{\mathcal{E}} H_{\mathcal{E}}^{q}(X, B)=$ $[d], d$ being the constant. We will say that the pair $(X, B)$ is of $\mathcal{E}$-finite type, or $\mathcal{E}$-finite for short, if $\operatorname{dim}_{\mathcal{E}} H_{\mathcal{E}}^{q}(X, B)$ is well-defined (in the above sense) and $\operatorname{dim}_{\mathcal{E}} H_{\mathcal{E}}^{q}(X, B)=[0]$ for almost all $q \in \mathbf{Z}$.

Suppose that $\Phi$ satisfies (PS)* and $\left(W, W^{-}\right)$is an admissible pair for $\Phi$ and $A:=\left\{p_{1}, \ldots, p_{k}\right\}$. We will say that $p_{j}$ is of $\mathcal{E}$-finite type, or $\mathcal{E}$-finite, if some (and therefore every) admissible pair for $\Phi$ and $p_{j}$ is $\mathcal{E}$-finite. If $\left(W, W^{-}\right)$and all $p_{j}$ are $\mathcal{E}$-finite, then we define

$$
M_{\mathcal{E}}^{q}\left(W, W^{-}\right):=\sum_{j=1}^{k} \operatorname{dim}_{\mathcal{E}} c_{\mathcal{E}}^{q}\left(\Phi, p_{j}\right), \quad q \in \mathbf{Z},
$$

and

$$
\beta_{\mathcal{E}}^{q}\left(W, W^{-}\right):=\operatorname{dim}_{\mathcal{E}} H_{\mathcal{E}}^{q}\left(W, W^{-}\right), \quad q \in \mathbf{Z} .
$$

Moreover, in such a case we may define the Morse and the Poincaré polynomials of $\left(W, W^{-}\right)$by setting

$$
M_{\mathcal{E}}\left(t, W, W^{-}\right):=\sum_{q=-\infty}^{\infty} M_{\mathcal{E}}^{q}\left(W, W^{-}\right) t^{q}
$$

and

$$
P_{\mathcal{E}}\left(t, W, W^{-}\right):=\sum_{q=-\infty}^{\infty} \beta_{\mathcal{E}}^{q}\left(W, W^{-}\right) t^{q} .
$$

Note that $M_{\mathcal{E}}$ and $P_{\mathcal{E}}$ are not polynomials in the usual sense (because some exponents $q$ may be negative). Formally, $M_{\mathcal{E}}$ and $P_{\mathcal{E}}$ are elements of $[\mathbf{Z}]\left[t, t^{-1}\right]$.

Theorem 3.1. (Morse inequalities) Suppose that $\Phi \in C^{1}(E, \mathbf{R})$ satisfies (PS)* and $\left(W, W^{-}\right)$is an admissible pair for $\Phi$ and $A:=\left\{p_{1}, \ldots, p_{k}\right\}$. If all $p_{j}$ are $\mathcal{E}$-finite, then the pair $\left(W, W^{-}\right)$is $\mathcal{E}$-finite and there is a polynomial $Q(t)=\sum_{q=-\infty}^{\infty} a_{q} t^{q}$ such that $a_{q} \in\left[\mathbf{Z}_{+}\right]$for all $q$ and

$$
M_{\mathcal{E}}\left(t, W, W^{-}\right)=P_{\mathcal{E}}\left(t, W, W^{-}\right)+(1+t) Q(t) .
$$

Note that an equivalent way of expressing the Morse inequalities is

$$
\sum_{j=-\infty}^{q}(-1)^{q-j} M_{\mathcal{E}}^{j}\left(W, W^{-}\right) \geq \sum_{j=-\infty}^{q}(-1)^{q-j} \beta_{\mathcal{E}}^{j}\left(W, W^{-}\right), \quad q \in \mathbf{Z} .
$$

First we prove the following special case of Theorem 3.1: 
Lemma 3.2. Under the hypotheses of Theorem 3.1, suppose that $\Phi\left(p_{1}\right)=\Phi\left(p_{2}\right)=$ $\ldots=\Phi\left(p_{k}\right)$. Then

$$
M_{\mathcal{E}}^{q}\left(W, W^{-}\right)=\beta_{\mathcal{E}}^{q}\left(W, W^{-}\right) \quad \text { for all } q \in \mathbf{Z} .
$$

Proof. Let $\left(W_{j}, W_{j}^{-}\right)$be an admissible pair for $\Phi$ and $p_{j}, 1 \leq j \leq k$. We may assume that the $W_{j}$ 's are pairwise disjoint. Then $\left(\bigcup_{j=1}^{k} W_{j}, \bigcup_{j=1}^{k} W_{j}^{-}\right)$is an admissible pair for $\Phi$ and $A$. Using Remark 2.11, we obtain

$$
H_{\mathcal{E}}^{q}\left(W, W^{-}\right) \cong H_{\mathcal{E}}^{q}\left(\bigcup_{j=1}^{k} W_{j}, \bigcup_{j=1}^{k} W_{j}^{-}\right) \cong \bigoplus_{j=1}^{k} H_{\mathcal{E}}^{q}\left(W_{j}, W_{j}^{-}\right)=\bigoplus_{j=1}^{k} c_{\mathcal{E}}^{q}\left(\Phi, p_{j}\right)
$$

So the conclusion follows from the definitions (3.1)-(3.3).

Proof of Theorem 3.1. Our argument follows closely [34] and [41].

Let $X \supset Y \supset Z$ be closed subsets of $E$. In the exact sequence of the triple $(X, Y, Z)$ (cf. (vi) of Proposition 1.3) denote the range of a mapping by $R$ and $\operatorname{dim}_{\mathcal{E}} H_{\mathcal{E}}^{q}($.$) by \beta_{\mathcal{E}}^{q}($.$) . Assume that the pairs (X, Y),(X, Z)$ and $(Y, Z)$ are $\mathcal{E}$-finite. It follows (using the exactness) that

$$
\begin{aligned}
\beta_{\mathcal{E}}^{q}(X, Z) & =\operatorname{dim}_{\mathcal{E}} R\left(j^{q}\right)+\operatorname{dim}_{\mathcal{E}} R\left(i^{q}\right), \\
\beta_{\mathcal{E}}^{q}(Y, Z) & =\operatorname{dim}_{\mathcal{E}} R\left(i^{q}\right)+\operatorname{dim}_{\mathcal{E}} R\left(\delta^{q}\right), \\
\beta_{\mathcal{E}}^{q}(X, Y) & =\operatorname{dim}_{\mathcal{E}} R\left(\delta^{q-1}\right)+\operatorname{dim}_{\mathcal{E}} R\left(j^{q}\right) .
\end{aligned}
$$

Hence

$$
\beta_{\mathcal{E}}^{q}(X, Y)+\beta_{\mathcal{E}}^{q}(Y, Z)=\beta_{\mathcal{E}}^{q}(X, Z)+\operatorname{dim}_{\mathcal{E}} R\left(\delta^{q-1}\right)+\operatorname{dim}_{\mathcal{E}} R\left(\delta^{q}\right) .
$$

Denote the Poincaré polynomial of $(X, Y)$ by $P_{\mathcal{E}}(t, X, Y)$, and set

$$
Q(t, X, Y, Z):=\sum_{q=-\infty}^{\infty} \operatorname{dim}_{\mathcal{E}} R\left(\delta^{q}\right) t^{q} .
$$

Since $\operatorname{dim}_{\mathcal{E}} R\left(\delta^{q}\right)=[0]$ for almost all $q$, it follows by multiplying (3.4) by $t^{q}$ and summing over $q$ that

$$
P_{\mathcal{E}}(t, X, Y)+P_{\mathcal{E}}(t, Y, Z)=P_{\mathcal{E}}(t, X, Z)+(1+t) Q(t, X, Y, Z) .
$$

Let $c_{1}<c_{2}<\ldots<c_{m}$ be the critical values of $\left.\Phi\right|_{W}$. Chooose numbers $d_{i}$ such that $d_{0}:=\inf _{W} \Phi, d_{m}:=\sup _{W} \Phi$ and

$$
d_{0}<c_{1}<d_{1}<c_{2}<\ldots<d_{m-1}<c_{m}<d_{m} .
$$

Define

$$
W_{i}:=\left(W \cap \Phi^{d_{i}}\right) \cup W^{-}, \quad i=0,1, \ldots, m,
$$

and, for all $i=1, \ldots, m$,

$$
\widetilde{W}_{i}:=\left\{x \in W_{i}: \Phi(x) \geq d_{i-1}\right\}, \quad \widetilde{W}_{i}^{-}:=\left\{x \in W_{i-1}: \Phi(x) \geq d_{i-1}\right\} .
$$

Note that $W_{m}=W, W_{0}=W^{-}$and

$$
H_{\mathcal{E}}^{*}\left(W_{i}, W_{i-1}\right) \stackrel{e x c}{\cong} H_{\mathcal{E}}^{*}\left(\widetilde{W}_{i}, \widetilde{W}_{i}^{-}\right), \quad i=1, \ldots, m .
$$


Since $\langle\nabla \Phi(x), V(x)\rangle>0$ whenever $x \in W \cap \Phi^{-1}\left(d_{i}\right)$ (cf. (iii) of Definition 2.1), it is easy to see that $\left(\widetilde{W}_{i}, \widetilde{W}_{i}^{-}\right)$is an admissible pair for $\Phi$ and the critical points $p_{j}$ satisfying $\Phi\left(p_{j}\right)=c_{i}$. Hence

$$
M_{\mathcal{E}}^{q}\left(W, W^{-}\right)=\sum_{i=1}^{m} M_{\mathcal{E}}^{q}\left(\widetilde{W_{i}}, \widetilde{W}_{i}^{-}\right)=\sum_{i=1}^{m} \beta_{\mathcal{E}}^{q}\left(\widetilde{W}_{i}, \widetilde{W}_{i}^{-}\right)
$$

according to Lemma 3.2 .

By (3.8), each pair $\left(\widetilde{W}_{i}, \widetilde{W}_{i}^{-}\right)$is $\mathcal{E}$-finite, and by $(3.7)$, the same is true for $\left(W_{i}, W_{i-1}\right)$. Exactness of the cohomology sequence of the triple $\left(W_{i}, W_{i-1}, W_{i-2}\right)$ implies that also the pair $\left(W_{i}, W_{i-2}\right)$ is $\mathcal{E}$-finite. So the $\mathcal{E}$-finiteness of $\left(W, W^{-}\right)=$ $\left(W_{m}, W_{0}\right)$ follows by induction.

Substituting $X=W_{m} \equiv W, Y=W_{i}$ and $Z=W_{i-1}$ in (3.6), we obtain

$$
P_{\mathcal{E}}\left(t, W, W_{i}\right)+P_{\mathcal{E}}\left(t, W_{i}, W_{i-1}\right)=P_{\mathcal{E}}\left(t, W, W_{i-1}\right)+(1+t) Q\left(t, W, W_{i}, W_{i-1}\right) .
$$

Adding these equalities gives

$$
\sum_{i=1}^{m} P_{\mathcal{E}}\left(t, W_{i}, W_{i-1}\right)=P_{\mathcal{E}}\left(t, W, W^{-}\right)+(1+t) Q(t),
$$

where $Q(t)$ has coefficients $a_{q} \in\left[\mathbf{Z}_{+}\right]$and $a_{q}=[0]$ for almost all $q$ (cf. (3.5)). Finally, multiplying (3.8) by $t^{q}$, summing over $q$ and employing (3.7), (3.9) and the definitions, we obtain

$$
\begin{aligned}
M_{\mathcal{E}}\left(t, W, W^{-}\right) & =\sum_{i=1}^{m} P_{\mathcal{E}}\left(t, \widetilde{W}_{i}, \widetilde{W}_{i}^{-}\right) \\
& =\sum_{i=1}^{m} P_{\mathcal{E}}\left(t, W_{i}, W_{i-1}\right)=P_{\mathcal{E}}\left(t, W, W^{-}\right)+(1+t) Q(t) .
\end{aligned}
$$

If $\Phi$ satisfies $(\mathrm{PS})^{*}, a<b, \Phi^{-1}(a), \Phi^{-1}(b)$ are bounded away from the critical set $K$ and $K \cap \Phi^{-1}(a, b)$ is finite, then $\left(W, W^{-}\right):=\left(\Phi^{-1}([a, b]), \Phi^{-1}(a)\right)$ is an admissible pair, as follows from Proposition 2.5. Since

$$
H_{\mathcal{E}}^{*}\left(W, W^{-}\right) \stackrel{e x c}{\cong} H_{\mathcal{E}}^{*}\left(\Phi^{b}, \Phi^{a}\right)
$$

we may denote the Morse and Poincaré polynomials of $\left(W, W^{-}\right)$by $M_{\mathcal{E}}\left(t, \Phi^{b}, \Phi^{a}\right)$ and $P_{\mathcal{E}}\left(t, \Phi^{b}, \Phi^{a}\right)$ in this case.

Corollary 3.3. Suppose that $\Phi \in C^{1}(E, \mathbf{R})$ satisfies $(P S)^{*}, \Phi^{-1}(a), \Phi^{-1}(b)$ (where $a<b)$ are bounded away from $K$ and the set $A:=K \cap \Phi^{-1}(a, b)$ is finite. If all points of $A$ are $\mathcal{E}$-finite, then

$$
M_{\mathcal{E}}\left(t, \Phi^{b}, \Phi^{a}\right)=P_{\mathcal{E}}\left(t, \Phi^{b}, \Phi^{a}\right)+(1+t) Q(t),
$$

where $Q(t)$ is as in Theorem 3.1.

Remark 3.4. Note for further reference that if $\left(E_{n}\right)_{n=1}^{\infty}$ is the trivial filtration of $E$ (i.e., $E_{n}=E$ for all $n$ ) and $d_{n}=0$ for all $n$, then essentially our theory is equivalent to the usual Morse theory. In particular, in this case our notion of admissible pair is a variant of the notion of Gromoll-Meyer pair as defined in [10]. 


\section{AuXiliary Results ON LINEAR MAPPINGS}

In order to study the local behaviour of a functional $\Phi$ near an isolated critical point $p$ one usually assumes that $\Phi$ satisfies (PS), the second Fréchet derivative of $\Phi$ exists, at least at $p$, and $L:=\Phi^{\prime \prime}(p)$ is a Fredholm operator $[10,34,41]$. Then $L$ is necessarily self-adjoint, of index 0 and

$$
E=R(L) \oplus N(L)
$$

where $R(L)$ and $N(L)$ are the range and the null space of $L$. Note that if $L$ is Fredholm, it is proper on bounded sets, i.e., if $C$ is compact, then the intersection of $L^{-1}(C)$ with any closed ball is compact. In other words, if $\left(x_{j}\right)$ is a bounded sequence such that $L x_{j} \rightarrow y$, then $\left(x_{j}\right)$ possesses a convergent subsequence.

If $\Phi$ satisfies (PS)* instead of (PS), it seems natural to replace the condition $L x_{j} \rightarrow y$ by $x_{j} \in E_{n_{j}}$ and $P_{n_{j}} L x_{j} \rightarrow y$, i.e., to assume that $L$ is A-proper. More precisely, let $E$ be a real Hilbert space with a given filtration $\mathcal{E}=\left(E_{n}\right)_{n=1}^{\infty}$. Recall that a mapping $f: D \rightarrow E, D$ a closed subset of $E$, is said to be $A$-proper (with respect to $\mathcal{E})$ if each bounded sequence $\left(x_{j}\right)_{j=1}^{\infty} \subset D$ such that $x_{j} \in D \cap E_{n_{j}}$ for some $n_{j}, n_{j} \rightarrow \infty$ and $P_{n_{j}} f\left(x_{j}\right) \rightarrow y \in E$ as $j \rightarrow \infty$, has a convergent subsequence. Clearly, if $x_{j_{k}} \rightarrow x$ as $k \rightarrow \infty$, then $f\left(x_{j_{k}}\right) \rightarrow f(x)$ and $f(x)=y$. It is easily seen that if $B$ is a compact mapping and $f(x)=x+B(x)$, then $f$ is A-proper. More generally, $f$ is A-proper if $f(x)=A x+B(x)$, where $A$ is a bounded linear Fredholm operator of index $0, A\left(E_{n}\right) \subset E_{n}$ for all $n$ and $B$ is compact. A survey of A-proper mappings may be found e.g. in Petryshyn $[35,36]$. The definition given there is more general than ours. On the other hand, in $[35,36]$ it is assumed that $\operatorname{dim} E_{n}<\infty$, which is not necessarily the case here.

In [36, Theorem II.3.1] it is shown that if $L$ is an A-proper bounded linear operator, then $L$ is Fredholm of index $\geq 0$. For the sake of completeness and because we do not assume that $\operatorname{dim} E_{n}<\infty$, we give a proof for a self-adjoint $L$.

Denote the space of bounded linear operators from $E$ to $F$ by $\mathcal{L}(E, F)$.

Proposition 4.1. If $L \in \mathcal{L}(E, E)$ is A-proper and self-adjoint, then $L$ is a Fredholm operator of index 0 .

Proof. In order to prove that $\operatorname{dim} N(L)<\infty$, assume the contrary. Then there exists a sequence $\left(x_{j}\right) \subset N(L)$ such that $\left\|x_{j}\right\|=1$ and $\left\|x_{i}-x_{j}\right\| \geq 1$ if $i \neq j$. For each $j$ there is a $z_{j} \in E_{n_{j}}, n_{j} \geq j$, such that $\left\|z_{j}-x_{j}\right\| \leq 1 / j$. Then $\left\|z_{i}-z_{j}\right\| \geq 1 / 2$ for all $i, j$ sufficiently large, $i \neq j$. On the other hand,

$$
\left\|P_{n_{j}} L z_{j}\right\|=\left\|P_{n_{j}} L\left(z_{j}-x_{j}\right)\right\| \leq \frac{1}{j}\|L\| \rightarrow 0,
$$

so $\left(z_{j}\right)$ has a convergent subsequence, a contradiction.

Since $L$ is self-adjoint,

$$
E=\overline{R(L)} \oplus N(L)
$$

To show that $R(L)$ is closed, let $y_{j} \rightarrow y$, where $y_{j} \in R(L)$. Then $y_{j}=L x_{j}$ for some $x_{j}$ and we may assume that $x_{j} \in \overline{R(L)}$. Again, there is a $z_{j} \in E_{n_{j}}, n_{j} \geq j$, such that $\left\|z_{j}-x_{j}\right\| \leq 1 / j$. Hence

$$
P_{n_{j}} L z_{j}=P_{n_{j}} L x_{j}+P_{n_{j}} L\left(z_{j}-x_{j}\right)=P_{n_{j}} y_{j}+P_{n_{j}} L\left(z_{j}-x_{j}\right) \rightarrow y .
$$

Therefore, if $\left(z_{j}\right)$ is bounded, $z_{j} \rightarrow z$ after passing to a subsequence, and $L z=y$. So $y \in R(L)$. If $\left(z_{j}\right)$ is unbounded, we may assume $\left\|z_{j}\right\| \rightarrow \infty$. Let $w_{j}:=z_{j} /\left\|z_{j}\right\|$. 
It follows from (4.1) that $P_{n_{j}} L w_{j} \rightarrow 0$, so after passing to a subsequence, $w_{j} \rightarrow w$ and $w \in N(L),\|w\|=1$. On the other hand, since $z_{j}-x_{j} \rightarrow 0$,

$$
\lim _{j \rightarrow \infty} \frac{x_{j}}{\left\|x_{j}\right\|}=\lim _{j \rightarrow \infty} w_{j}=w .
$$

Hence $w \in \overline{R(L)}$. This contradiction completes the proof of the closedness of $R(L)$.

We have shown that $L$ is a Fredholm operator. Since it is self-adjoint, its index must be 0 .

Assume from now on that $L \in \mathcal{L}(E, E)$ is a self-adjoint Fredholm operator (of index 0$)$ and $\mathcal{E}$ is a given filtration. As usual, $P_{n}$ is the orthogonal projector of $E$ onto $E_{n}$.

Lemma 4.2. (i) There exists an $n_{0}$ such that if $n \geq n_{0}$, then $\left.P_{n}\right|_{N(L)}: N(L) \rightarrow$ $P_{n} N(L)$ is a linear isomorphism and $\|z\| \leq 2\left\|P_{n} z\right\|$ for all $z \in N(L)$.

(ii) $E_{n}=\left(R(L) \cap E_{n}\right) \oplus P_{n} N(L)$, and the spaces $R(L) \cap E_{n}$ and $P_{n} N(L)$ are orthogonal.

Proof. (i) This is obvious because $\operatorname{dim} N(L)<\infty$ and $P_{n} \rightarrow I$ uniformly on compact sets ( $I$ is the identity operator).

(ii) Let $x \in E_{n}$ and suppose that $x$ is orthogonal to $P_{n} N(L)$. Then

$$
0=\left\langle x, P_{n} z\right\rangle=\langle x, z\rangle \quad \forall z \in N(L) .
$$

It follows that $x \in R(L)$, so the orthogonal complement of $P_{n} N(L)$ in $E_{n}$ is $R(L) \cap$ $E_{n}$.

Let $Q_{n}$ be the orthogonal projector of $R(L)$ onto $R(L) \cap E_{n}$. Since $\left.P_{n}\right|_{R(L)}$ and $Q_{n}$ map $R(L)$ into $E, P_{n}-Q_{n}$ may be considered as an element of $\mathcal{L}(R(L), E)$.

Proposition 4.3. $P_{n}-Q_{n} \rightarrow 0$ in $\mathcal{L}(R(L), E)$ as $n \rightarrow \infty$.

Proof. Let $x \in R(L)$. Since $P_{n} y=Q_{n} y=y$ whenever $y \in R(L) \cap E_{n}$,

$$
\left\langle P_{n} x-Q_{n} x, y\right\rangle=0 \quad \forall y \in R(L) \cap E_{n} .
$$

Therefore, in view of (ii) of Lemma 4.2, $P_{n} x-Q_{n} x \in P_{n} N(L)$. So $P_{n} x-Q_{n} x=P_{n} z_{n}$ for some $z_{n} \in N(L)$. By (i) of Lemma 4.2,

$$
\left\|z_{n}\right\| \leq 2\left\|P_{n} z_{n}\right\|=2\left\|\left(P_{n}-Q_{n}\right) x\right\| \leq 4\|x\|
$$

for almost all $n$. Since $x \in R(L), Q_{n} x \in R(L) \cap E_{n}$ and $z_{n} \in N(L)$,

$$
\begin{aligned}
\left\|\left(P_{n}-Q_{n}\right) x\right\|^{2} & =\left\langle P_{n} z_{n},\left(P_{n}-Q_{n}\right) x\right\rangle \\
& =\left\langle P_{n} z_{n}, x\right\rangle=\left\langle P_{n} z_{n}-z_{n}, x\right\rangle \leq\left\|\left(P_{n}-I\right) z_{n}\right\|\|x\| .
\end{aligned}
$$

Since $P_{n} \rightarrow I$ uniformly on bounded subsets of $N(L)$, it follows from (4.2) and (4.3) that $\left(P_{n}-Q_{n}\right) x \rightarrow 0$ as $n \rightarrow \infty$, uniformly in $x \in R(L),\|x\| \leq 1$. Hence the conclusion.

Corollary 4.4. The sequence $\left(R(L) \cap E_{n}\right)_{n=1}^{\infty}$ is a filtration of $R(L)$. More precisely, for each $x \in R(L), Q_{n} x \rightarrow x$ as $n \rightarrow \infty$.

Proof. Immediate from Proposition 4.3 since $P_{n} x \rightarrow x$ as $n \rightarrow \infty$. 
Theorem 4.5. Let $L \in \mathcal{L}(E, E)$ be a self-adjoint operator. Then the following conditions are equivalent:

(i) $L$ is A-proper;

(ii) $L$ is a Fredholm operator of index 0 and there exist $c>0$ and $n_{0} \geq 1$ such that if $n \geq n_{0}$, then $\left\|P_{n} L x\right\| \geq c\|x\|$ for all $x \in R(L) \cap E_{n}$.

Proof. (i) $\Rightarrow$ (ii) By Proposition 4.1, $L$ is Fredholm of index 0. Suppose that for any $j \geq 1$ there exist $n_{j} \geq j$ and $x_{n_{j}} \in R(L) \cap E_{n_{j}}$ such that

$$
\left\|P_{n_{j}} L x_{n_{j}}\right\|<\frac{1}{j}\left\|x_{n_{j}}\right\| \text {. }
$$

Then $P_{n_{j}} L y_{n_{j}} \rightarrow 0$, where $y_{n_{j}}:=x_{n_{j}} /\left\|x_{n_{j}}\right\|$. The A-properness of $L$ implies that, after passing to a subsequence, $y_{n_{j}} \rightarrow y \in R(L)$ and $\|y\|=1$. But $L y=0$, i.e., $y \in N(L)$. This contradiction shows that (ii) is satisfied.

(ii) $\Rightarrow$ (i) Although this implication will not be used, we prove it for the sake of completeness.

Suppose that $L$ is Fredholm of index 0 . Let $\left(x_{j}\right) \subset E$ be a bounded sequence such that $x_{j} \in E_{n_{j}}, n_{j} \rightarrow \infty$ and $P_{n_{j}} L x_{j} \rightarrow y$ as $j \rightarrow \infty$. Passing to a subsequence, $x_{j} \rightarrow x$ weakly and $P_{n_{j}} L x_{j} \rightarrow L x$ weakly. Therefore $y=L x$ and $y \in R(L)$. According to (ii) of Lemma $4.2, x_{j}=u_{j}+P_{n_{j}} z_{j}$, where $u_{j} \in R(L) \cap E_{n_{j}}$ and $z_{j} \in N(L)$. Passing to a subsequence again, $z_{j} \rightarrow z$ and

$$
P_{n_{j}} L u_{j}=P_{n_{j}} L x_{j}-P_{n_{j}} L P_{n_{j}} z_{j} \rightarrow y+L z=y .
$$

There is a $u \in R(L)$ such that $L u=y$. Since $u_{j}-Q_{n_{j}} u \in R(L) \cap E_{n_{j}}$, it follows from the inequality in (ii) and Corollary 4.4 that

$$
c\left\|u_{j}-Q_{n_{j}} u\right\| \leq\left\|P_{n_{j}} L\left(u_{j}-Q_{n_{j}} u\right)\right\|=\left\|P_{n_{j}} L u_{j}-P_{n_{j}} L Q_{n_{j}} u\right\| \rightarrow\|y-L u\|=0 .
$$

Hence $u_{j} \rightarrow u$, and therefore $x_{j}=u_{j}+P_{n_{j}} z_{j} \rightarrow u+z$. This shows that $L$ is A-proper.

\section{Computation of CRitical groups}

Suppose $\left(E_{n}\right)_{n=1}^{\infty}$ is a filtration of a real Hilbert space $E$ and $\mathcal{E}=\left\{E_{n}, d_{n}\right\}_{n=1}^{\infty}$.

For an arbitrary self-adjoint operator $L \in \mathcal{L}(E, E)$, denote the Morse index of $L$ (or more precisely, of the quadratic form $x \mapsto\langle L x, x\rangle$ ) by $M^{-}(L)$. Suppose $L$ is a Fredholm operator, and recall that

$$
Q_{n}: R(L) \rightarrow R(L) \cap E_{n}
$$

is the orthogonal projector of $R(L)$ onto $R(L) \cap E_{n}$. Define the $\mathcal{E}$-Morse index $M_{\mathcal{E}}^{-}(L)$ of $L$ by the formula

$$
M_{\mathcal{E}}^{-}(L):=\lim _{n \rightarrow \infty}\left(M^{-}\left(\left.Q_{n} L\right|_{R(L) \cap E_{n}}\right)-d_{n}\right) .
$$

The above limit exists in many important cases, as will be shown later. However, in general it does not, so $M_{\mathcal{E}}^{-}(L)$ is not always well-defined.

As usual, $M^{0}(L)$ will denote the nullity of $L$, i.e.,

$$
M^{0}(L):=\operatorname{dim} N(L) .
$$

Observe that $M^{-}\left(\left.Q_{n} L\right|_{R(L) \cap E_{n}}\right)$ is the Morse index of the quadratic form $R(L) \cap$ $E_{n} \ni x \mapsto\langle L x, x\rangle$. It might seem more natural to consider the form $E_{n} \ni x \mapsto$ $\langle L x, x\rangle$ and therefore define the $\mathcal{E}$-Morse index by

$$
\widetilde{M}_{\mathcal{E}}^{-}(L):=\lim _{n \rightarrow \infty}\left(M^{-}\left(\left.P_{n} L\right|_{E_{n}}\right)-d_{n}\right) .
$$


However, in view of Lemma 4.2, $E_{n}=\left(R(L) \cap E_{n}\right) \oplus P_{n} N(L)$, and since the quadratic form $P_{n} N(L) \ni x \mapsto\langle L x, x\rangle$ tends to 0 as $n \rightarrow \infty$ (in the sense that $|\langle L x, x\rangle| \leq \varepsilon_{n}\|x\|^{2}$, where $\left.\varepsilon_{n} \rightarrow 0\right)$, its contribution to the Morse index as $n \rightarrow \infty$ should be neglected. This justifies the definition (5.1). See also Theorem 5.4.

Remark 5.1. If $N(L) \subset E_{n}$, then $E_{n}=\left(R(L) \cap E_{n}\right) \oplus N(L)$, and the quadratic forms $\left(R(L) \cap E_{n}\right) \ni x \mapsto\langle L x, x\rangle$ and $E_{n} \ni x \mapsto\langle L x, x\rangle$ have the same Morse index. Therefore if $N(L) \subset E_{n_{0}}$ for some $n_{0}$, then $M_{\mathcal{E}}^{-}(L)$ is well-defined if and only if $\widetilde{M}_{\mathcal{E}}^{-}(L)$ is and $M_{\mathcal{E}}^{-}(L)=\widetilde{M}_{\mathcal{E}}^{-}(L)$.

Proposition 5.2. Suppose $A \in \mathcal{L}(E, E)$ is a self-adjoint Fredholm operator of index 0 such that $A\left(E_{n}\right) \subset E_{n}$ for almost all $n$ and $B \in \mathcal{L}(E, E)$ is a self-adjoint compact operator. Then $A+B$ is A-proper. If $M^{-}\left(\left.A\right|_{E_{n}}\right)=d_{n}+k$ for almost all $n$ and some $k \in \mathbf{Z}$, then $M_{\mathcal{E}}^{-}(A+B)$ is well-defined and finite.

Proof. Set $L:=A+B$. It is easy to verify that $L$ is A-proper.

Let us introduce some auxiliary notation. $Q: E \rightarrow R(L)$ is the orthogonal projector,

$$
F_{n}:=\left(R(L) \cap E_{n+1}\right) \cap\left(R(L) \cap E_{n}\right)^{\perp}, \quad G_{n}:=E_{n+1} \cap E_{n}^{\perp}
$$

and

$$
U_{n}:=P_{n+1}-P_{n}: E \rightarrow G_{n} .
$$

According to Lemma 4.2, $E_{n}=\left(R(L) \cap E_{n}\right) \oplus P_{n} N(L)$ and $E_{n+1}=\left(R(L) \cap E_{n+1}\right) \oplus$ $P_{n+1} N(L)$. Therefore

$$
E_{n+1}=\left(R(L) \cap E_{n}\right) \oplus F_{n} \oplus P_{n+1} N(L)=\left(R(L) \cap E_{n}\right) \oplus P_{n} N(L) \oplus G_{n}
$$

and the sums are orthogonal. So each $x \in R(L) \cap E_{n+1}$ may be represented as

$$
x=y+z=y+P_{n} \zeta+w,
$$

where $y \in R(L) \cap E_{n}, z \in F_{n}, \zeta \in N(L)$ and $w \in G_{n}$.

It follows from Proposition 4.3 and Theorem 4.5 that there exist $\varepsilon>0$ and $m_{0} \geq 1$ such that

$$
\left\|Q_{n} L x\right\| \geq 2 \varepsilon\|x\| \quad \text { whenever } x \in R(L) \cap E_{n} \text { and } n \geq m_{0} .
$$

For $n \geq m_{0}$ and $x \in R(L) \cap E_{n+1}$, define

$$
T_{n} x:=Q_{n+1} L x-\left(Q_{n} L Q_{n} x+U_{n} A U_{n} x\right) .
$$

Using the decomposition (5.3) of $x$ and observing that $\langle A y, w\rangle=0$ (because $\left.A\left(E_{n}\right) \subset E_{n}\right)$, we obtain

$$
\begin{aligned}
\left\langle T_{n} x, x\right\rangle & =\langle L(y+z), y+z\rangle-\langle L y, y\rangle-\langle A w, w\rangle \\
& =2\left\langle L(y+w), P_{n} \zeta\right\rangle+\left\langle L P_{n} \zeta, P_{n} \zeta\right\rangle+2\langle B y, w\rangle+\langle B w, w\rangle .
\end{aligned}
$$

Since $B$ is compact, $w \in G_{n} \subset E_{n}^{\perp}$ and $L P_{n} \zeta \rightarrow 0$ uniformly in $\zeta \in N(L) \cap \bar{B}(0,1)$, it follows that $\left\|T_{n}\right\|=\sup \left\{\left|\left\langle T_{n} x, x\right\rangle\right|: x \in R(L) \cap E_{n+1} \cap \bar{B}(0,1)\right\} \leq \varepsilon$ if $m_{0}$ is large enough. Hence by (5.4),

$$
\left\|Q_{n+1} L x-\lambda T_{n} x\right\| \geq\left\|Q_{n+1} L x\right\|-\lambda\left\|T_{n} x\right\| \geq \varepsilon\|x\|
$$

for each $\lambda \in[0,1]$ and $x \in R(L) \cap E_{n+1}$. Therefore, for $n \geq m_{0}$,

$$
\begin{aligned}
M^{-}\left(\left.Q_{n+1} L\right|_{R(L) \cap E_{n+1}}\right) & =M^{-}\left(\left.\left(Q_{n} L Q_{n}+U_{n} A U_{n}\right)\right|_{R(L) \cap E_{n+1}}\right) \\
& =M^{-}\left(\left.Q_{n} L\right|_{R(L) \cap E_{n}}\right)+M^{-}\left(\left.A\right|_{G_{n}}\right) .
\end{aligned}
$$


The second equality follows because $R(L) \cap E_{n}$ is orthogonal to $G_{n}$. Finally, since $A\left(E_{n}\right) \subset E_{n}$,

$$
d_{n+1}-d_{n}=M^{-}\left(\left.A\right|_{E_{n+1}}\right)-M^{-}\left(\left.A\right|_{E_{n}}\right)=M^{-}\left(\left.A\right|_{G_{n}}\right) .
$$

This and (5.5) imply that $M^{-}\left(\left.Q_{n} L\right|_{R(L) \cap E_{n}}\right)-d_{n}$ is constant for almost all $n$. Moreover, it is finite because $M^{-}\left(\left.Q_{n} Q A\right|_{R(L) \cap E_{n}}\right) \leq d_{n}+k, \operatorname{dim} N(A)<\infty$ and $B$ is compact.

Theorem 5.3. Suppose that $\Phi \in C^{1}(E, \mathbf{R}), p$ is an isolated critical point of $\Phi$ and

$$
\Phi(x)=\Phi(p)+\frac{1}{2}\langle L(x-p), x-p\rangle+\varphi(x),
$$

where $L$ is an invertible A-proper operator and $\nabla \varphi(x)=o(\|x-p\|)$ as $x \rightarrow p$. If $M_{\mathcal{E}}^{-}(L)$ is well-defined and finite, then $c_{\mathcal{E}}^{q}(\Phi, p)=[\mathcal{F}]$ for $q=M_{\mathcal{E}}^{-}(L)$ and $[0]$ otherwise. If $M_{\mathcal{E}}^{-}(L)=+\infty$ or $-\infty$, then $c_{\mathcal{E}}^{q}(\Phi, p)=[0]$ for all $q$.

Proof. Note first that since $R(L)=E$, we have $P_{n}=Q_{n}$ and $M_{\mathcal{E}}^{-}(L)=\widetilde{M}_{\mathcal{E}}^{-}(L)$. Consider the family of functionals

$$
\Phi_{\lambda}(x):=\Phi(p)+\frac{1}{2}\langle L(x-p), x-p\rangle+(1-\lambda) \varphi(x), \quad \lambda \in[0,1], x \in E .
$$

By Theorem 4.5, there are $c>0$ and $n_{0} \geq 1$ such that for $n \geq n_{0}$ and $x \in E_{n}$, $\left\|P_{n} L x\right\| \geq c\|x\|$. Take $\delta>0$ such that $\|\nabla \varphi(x)\| \leq \frac{c}{2}\|x-p\|$ for $x \in B(p, \delta)$. We claim that each $\Phi_{\lambda}$ satisfies (PS)* on $\bar{B}(p, \delta)$. Indeed, assume $x_{k} \in \bar{B}(p, \delta) \cap E_{n_{k}}$, $n_{k} \rightarrow \infty$ and $P_{n_{k}} \nabla \Phi_{\lambda}\left(x_{k}\right) \rightarrow 0$. Let $p_{k}:=P_{n_{k}} p \in E_{n_{k}}$. Since $\nabla \Phi_{\lambda}\left(x_{k}\right)=$ $L\left(x_{k}-p_{k}\right)+L\left(p_{k}-p\right)+(1-\lambda) \nabla \varphi\left(x_{k}\right)$,

$$
\begin{aligned}
\left\|P_{n_{k}} \nabla \Phi_{\lambda}\left(x_{k}\right)\right\| & \geq\left\|P_{n_{k}} L\left(x_{k}-p_{k}\right)\right\|-\left\|L\left(p_{k}-p\right)\right\|-\left\|\nabla \varphi\left(x_{k}\right)\right\| \\
& \geq c\left\|x_{k}-p_{k}\right\|-\left\|L\left(p_{k}-p\right)\right\|-\frac{c}{2}\left\|x_{k}-p\right\|
\end{aligned}
$$

for $n_{k} \geq n_{0}$. Hence $x_{k} \rightarrow p$ as $k \rightarrow \infty$.

In view of Corollary 2.9,

$$
c_{\mathcal{E}}^{*}\left(\Phi_{1}, p\right)=c_{\mathcal{E}}^{*}(\Phi, p) .
$$

Since $\Phi_{1}(x)=\Phi(p)+\frac{1}{2}\langle L(x-p), x-p\rangle$ clearly satisfies the hypotheses of Corollary 2.10 , we may assume that $p=0$ (and of course that $\Phi_{1}(0)=0$ ).

Let $W:=\Phi_{1}^{-1}([-1,1])$ and $W^{-}:=\Phi_{1}^{-1}(-1)$. Since $\Phi_{1}$ satisfies (PS)* on $E$, $\left(W, W^{-}\right)$is an admissible pair for $\Phi_{1}$ and 0 (see Proposition 2.5). Moreover, if $n \geq n_{0}$, then $\left.\Phi_{1}\right|_{E_{n}}$ is a nondegenerate quadratic form and $\left(W \cap E_{n}, W^{-} \cap E_{n}\right)$ is an admissible pair for $\left.\Phi_{1}\right|_{E_{n}}$ and 0 (with respect to the trivial filtration of $E_{n}$, cf. Remark 3.4).

It remains to compute $H^{*}\left(W \cap E_{n}, W^{-} \cap E_{n}\right)$. Choose an equivalent inner product in $E_{n}$ such that $\Phi_{1}(x)=\frac{1}{2}\left\|x^{+}\right\|^{2}-\frac{1}{2}\left\|x^{-}\right\|^{2}$, where $x=x^{+}+x^{-} \in E_{n}^{+} \oplus$ $E_{n}^{-}=E_{n}$. For $x \in \Phi_{1}^{1} \cap E_{n}$, let $t(x)$ be the smallest $t \geq 0$ for which $x^{+}+(1-t) x^{-} \in$ $\Phi_{1}^{-1}([-1,1])$. Then the mapping $(\lambda, x) \mapsto x^{+}+(1-\lambda t(x)) x^{-}, \lambda \in[0,1]$, is a strong deformation retraction of $\left(\Phi_{1}^{1} \cap E_{n}, \Phi_{1}^{-1} \cap E_{n}\right) \equiv\left(\left\{x \in E_{n}: \Phi_{1}(x) \leq 1\right\},\{x \in\right.$ $\left.\left.E_{n}: \Phi_{1}(x) \leq-1\right\}\right)$ onto $\left(W \cap E_{n}, W^{-} \cap E_{n}\right)$. Similarly, the mapping $(\lambda, x) \mapsto$ $(1-\lambda) x^{+}+x^{-}, \lambda \in[0,1]$, is a strong deformation retraction of $\left(\Phi_{1}^{1} \cap E_{n}, \Phi_{1}^{-1} \cap E_{n}\right)$ onto $\left(\Phi_{1}^{1} \cap E_{n}^{-}, \Phi_{1}^{-1} \cap E_{n}^{-}\right)=\left(E_{n}^{-}, E_{n}^{-}-B(0,1)\right)$. Hence $\left(W \cap E_{n}, W^{-} \cap E_{n}\right)$ is homotopy equivalent to $(B, \partial B)$, where $B$ is the closed unit ball in $E_{n}^{-}$, and therefore $H^{*}\left(W \cap E_{n}, W^{-} \cap E_{n}\right) \cong H^{*}(B, \partial B)$. Since $\operatorname{dim} E_{n}^{-}=M^{-}\left(\left.P_{n} L\right|_{E_{n}}\right)$, we 
obtain $H^{q+d_{n}}\left(W \cap E_{n}, W^{-} \cap E_{n}\right) \cong \mathcal{F}$ if $q=M^{-}\left(\left.P_{n} L\right|_{E_{n}}\right)-d_{n}$ and 0 otherwise. Now the conclusion follows from the definitions of $H_{\mathcal{E}}^{*}$ and $M_{\mathcal{E}}^{-}(L)$.

Suppose $\Phi \in C^{2}(U, \mathbf{R})$, where $U$ is a neighbourhood of a critical point $p$. Then $\Phi$ admits the representation (5.6). Assume that the operator $L$ is Fredholm, let $x=p+z+y$, where $z \in N(L), y \in R(L)$, and denote the orthogonal projector onto $R(L)$ by $Q$. Then

$$
\begin{gathered}
\nabla \Phi(p+z+y)=L y+\nabla \varphi(p+z+y) \\
\nabla \Phi(p)=0 \quad \text { and } \quad \Phi^{\prime \prime}(p)=L .
\end{gathered}
$$

Since $\left.L\right|_{R(L)}$ is invertible, it follows from the implicit function theorem that there exist $\delta>0$ and a $C^{1}$-function $y=\alpha(z): B(0, \delta) \cap N(L) \rightarrow R(L)$ such that $\alpha(0)=0$, $\alpha^{\prime}(0)=0$ and

$$
Q \nabla \Phi(p+z+\alpha(z)) \equiv 0
$$

Define

$$
\widetilde{\varphi}(z):=\Phi(p+z+\alpha(z))-\Phi(p)=\frac{1}{2}\langle L \alpha(z), \alpha(z)\rangle+\varphi(p+z+\alpha(z)) .
$$

Suppose 0 is an isolated critical point of $\widetilde{\varphi}$ and let $c^{q}(\widetilde{\varphi}, 0):=H^{q}\left(\widetilde{W}, \widetilde{W}^{-}\right)$, where $\left(\widetilde{W}, \widetilde{W}^{-}\right)$is an admissible pair for $\widetilde{\varphi}$ and 0 in $N(L)$ (with respect to the trivial filtration of $N(L))$.

Theorem 5.4. Suppose $U$ is a neighbourhood of an isolated critical point $p$ of $\Phi \in C^{2}(U, \mathbf{R})$ and the operator $L(c f .(5.6))$ is A-proper. If $M_{\mathcal{E}}^{-}(L)$ is well-defined and finite, then $c_{\mathcal{E}}^{q}(\Phi, p) \cong\left[c^{q-M_{\mathcal{E}}^{-}(L)}(\widetilde{\varphi}, 0)\right]$ for all $q(\widetilde{\varphi}$ is given by the formula (5.8)). If $M_{\mathcal{E}}^{-}(L)=+\infty$ or $-\infty$, then $c_{\mathcal{E}}^{q}(\Phi, p)=[0]$ for all $q$.

Proof. Consider a family of functionals

$$
\begin{gathered}
\Phi_{\lambda}(p+z+y):=\Phi(p)+\frac{1}{2}\langle L y, y\rangle+\frac{1}{2} \lambda(2-\lambda)\langle L \alpha(z), \alpha(z)\rangle \\
+\lambda \varphi(p+z+\alpha(z))+(1-\lambda) \varphi(p+z+y+\lambda \alpha(z))
\end{gathered}
$$

where $z+y \in(N(L) \oplus R(L)) \cap B(0, \delta)$ and $0 \leq \lambda \leq 1$ (this family has been introduced by Dancer in [15]). Observe that $\Phi_{0}=\Phi$,

$$
\Phi_{1}(p+z+y)=\Phi(p)+\frac{1}{2}\langle L y, y\rangle+\widetilde{\varphi}(z)
$$

and

$$
\begin{aligned}
& \nabla_{y} \Phi_{\lambda}(p+z+y)=L y+(1-\lambda) Q \nabla \varphi(p+z+y+\lambda \alpha(z)), \\
& \nabla_{z} \Phi_{\lambda}(p+z+y) \\
& =\lambda(2-\lambda)\left\langle L \alpha(z), \alpha^{\prime}(z) \cdot\right\rangle+\lambda\left\langle\nabla \varphi(p+z+\alpha(z)), \cdot+\alpha^{\prime}(z) \cdot\right\rangle \\
& \quad+(1-\lambda)\left\langle\nabla \varphi(p+z+y+\lambda \alpha(z)), \cdot+\lambda \alpha^{\prime}(z) \cdot\right\rangle .
\end{aligned}
$$

We will show that the family $\left\{\Phi_{\lambda}\right\}$ satisfies the hypotheses of Corollary 2.9 on a suitably small ball $B(p, r)$, where $0<r<\delta$ and $K\left(\Phi_{\lambda}\right) \cap B(p, r)=\{p\}$. 
First we verify that each $\Phi_{\lambda}$ satisfies (PS)* on $\bar{B}(p, r)$. Suppose $x_{k}=p+z_{k}+y_{k} \in$ $\bar{B}(p, r) \cap E_{n_{k}}, n_{k} \rightarrow \infty$ and $P_{n_{k}} \nabla \Phi_{\lambda}\left(x_{k}\right) \rightarrow 0$. Then, in view of (5.9),

$P_{n_{k}} \nabla_{y} \Phi_{\lambda}\left(p+z_{k}+y_{k}\right)=P_{n_{k}} L y_{k}+(1-\lambda) P_{n_{k}} Q \nabla \varphi\left(p+z_{k}+y_{k}+\lambda \alpha\left(z_{k}\right)\right)=: w_{k} \rightarrow 0$.

Setting $v_{k}:=y_{k}-(1-\lambda) \alpha\left(z_{k}\right)$, we get

$$
(1-\lambda) P_{n_{k}} L \alpha\left(z_{k}\right)+P_{n_{k}} L v_{k}+(1-\lambda) P_{n_{k}} Q \nabla \varphi\left(p+z_{k}+\alpha\left(z_{k}\right)+v_{k}\right)=w_{k} .
$$

Since $L \alpha\left(z_{k}\right)+Q \nabla \varphi\left(p+z_{k}+\alpha\left(z_{k}\right)\right)=0$ (cf. (5.7)),

$$
P_{n_{k}} L v_{k}+(1-\lambda) P_{n_{k}} Q\left(\nabla \varphi\left(p+z_{k}+\alpha\left(z_{k}\right)+v_{k}\right)-\nabla \varphi\left(p+z_{k}+\alpha\left(z_{k}\right)\right)\right)=w_{k} .
$$

Since $v_{k}=x_{k}-p-z_{k}-(1-\lambda) \alpha\left(z_{k}\right)$ and $x_{k} \in E_{n_{k}}$,

$$
P_{n_{k}} v_{k}-v_{k}=\left(I-P_{n_{k}}\right)\left(p+z_{k}+(1-\lambda) \alpha\left(z_{k}\right)\right) \text {. }
$$

So $P_{n_{k}} v_{k}-v_{k} \rightarrow 0$ (because $N(L)$ is finite dimensional) and $Q_{n_{k}} v_{k}-v_{k} \rightarrow 0$ according to Proposition 4.3. It follows from Theorem 4.5 that

$$
\begin{aligned}
& \left\|P_{n_{k}} L v_{k}\right\| \geq\left\|P_{n_{k}} L Q_{n_{k}} v_{k}\right\|-\left\|P_{n_{k}} L\left(Q_{n_{k}} v_{k}-v_{k}\right)\right\| \\
& \quad \geq c\left\|Q_{n_{k}} v_{k}\right\|-\|L\|\left\|Q_{n_{k}} v_{k}-v_{k}\right\| \geq c\left\|v_{k}\right\|-(\|L\|+c)\left\|Q_{n_{k}} v_{k}-v_{k}\right\|
\end{aligned}
$$

for almost all $k$. Since $\varphi \in C^{2}(U, \mathbf{R})$ and $\varphi^{\prime \prime}(p)=0$, then taking the radius $r$ of the ball $B(p, r)$ smaller if necessary, we obtain

$$
\left\|\nabla \varphi\left(p+z_{k}+\alpha\left(z_{k}\right)+v_{k}\right)-\nabla \varphi\left(p+z_{k}+\alpha\left(z_{k}\right)\right)\right\| \leq \frac{c}{2}\left\|v_{k}\right\| .
$$

Combining this with (5.11) and (5.12) gives

$$
c\left\|v_{k}\right\|-(\|L\|+c)\left\|Q_{n_{k}} v_{k}-v_{k}\right\| \leq \frac{c}{2}\left\|v_{k}\right\|+\left\|w_{k}\right\| .
$$

Hence $v_{k} \rightarrow 0$ as $k \rightarrow \infty$. Passing to a subsequence, $z_{k} \rightarrow \bar{z}$ and $x_{k}=p+z_{k}+y_{k} \rightarrow$ $p+\bar{z}+(1-\lambda) \alpha(\bar{z})$. This completes the proof of (PS)*.

Suppose $p+z+y \in B(p, r)$ and $\nabla \Phi_{\lambda}(p+z+y)=0$. Since $\left.Q \Phi_{\lambda}^{\prime \prime}(p)\right|_{R(L)}=\left.L\right|_{R(L)}$ (cf. (5.9)), it follows from the implicit function theorem that (5.9) has a unique solution $y=y(z, \lambda)$ provided $r$ is small enough $(r$ independent of $\lambda)$. A direct verification using (5.7) shows that $y=(1-\lambda) \alpha(z)$. Inserting this in (5.10) we obtain

$$
\begin{aligned}
& \nabla_{z} \Phi_{\lambda}(p+z+(1-\lambda) \alpha(z)) \\
& \quad=\lambda(2-\lambda)\left\langle\nabla \Phi(p+z+\alpha(z)), \alpha^{\prime}(z) \cdot\right\rangle+(I-Q) \nabla \varphi(p+z+\alpha(z)) \\
& \quad=(I-Q) \nabla \varphi(p+z+\alpha(z))
\end{aligned}
$$

because $\alpha^{\prime}(z) \cdot \in R(L)$ and (5.7) is satisfied. Recall that $K(\Phi) \cap B(p, r)=\{p\}$. Since $\nabla \Phi(p+z+y)=0$ if and only if $y=\alpha(z)$ and $(I-Q) \nabla \varphi(p+z+\alpha(z))=0$, we must have $z=0$ and $y=(1-\lambda) \alpha(0)=0$. So $K\left(\Phi_{\lambda}\right) \cap B(p, r)=\{p\}$.

Since $\nabla \varphi$ is locally Lipschitz continuous at $p$, it is easy to see from (5.9), (5.10) that if $r$ is small enough, then $\left|\Phi_{\lambda}(x)\right|$ is bounded by a constant independent of $x \in B(p, r)$ and $\lambda \in[0,1]$, and the mapping $\lambda \mapsto \nabla \Phi_{\lambda}$ is continuous, uniformly in $x \in B(p, r)$. Now all the hypotheses of Corollary 2.9 are verified; hence

$$
c_{\mathcal{E}}^{*}(\Phi, p) \cong c_{\mathcal{E}}^{*}\left(\Phi_{1}, p\right) .
$$

Moreover, by Corollary 2.10, we may assume that $p=0$ (and $\Phi(p)=0$ ). 
Let $\chi(y):=\frac{1}{2}\langle L y, y\rangle$. Then $\Phi_{1}(z+y)=\chi(y)+\widetilde{\varphi}(z)$. Since $\left(R(L) \cap E_{n}\right)_{n=1}^{\infty}$ is a filtration of $R(L)$ (cf. Corollary 4.4), there exists an admissible pair $\left(W_{1}, W_{1}^{-}\right)$for $\chi$ and 0 (in $R(L)$ ) such that $W_{1}$ is bounded. Denote the corresponding admissible field by $V_{1}(y)$. Let $\left(\widetilde{W}, \widetilde{W}^{-}\right)$be an admissible pair for $\widetilde{\varphi}$ and $0, \widetilde{W} \subset B(0, \delta) \cap N(L)$, and let $\widetilde{V}(z)$ be a corresponding admissible field. Choose $m_{0}$ so that $\left.P_{m_{0}}\right|_{N(L)}$ : $N(L) \rightarrow P_{m_{0}} N(L)$ is a linear isomorphism, and define

$$
W_{2}:=P_{m_{0}} \widetilde{W}, \quad W_{2}^{-}:=P_{m_{0}} \widetilde{W}^{-}
$$

and

$$
\left(W, W^{-}\right):=\left(W_{1}+W_{2},\left(W_{1}^{-}+W_{2}\right) \cup\left(W_{1}+W_{2}^{-}\right)\right) .
$$

Note that $W_{1} \cap W_{2}=\{0\}$ since $W_{1} \subset R(L)$ and $W_{2} \subset P_{m_{0}} N(L)$. We claim that $\left(W, W^{-}\right)$is an admissible pair for $\Phi_{1}$ and 0 . For each $x \in E$ we have the unique decompositions $x=z+y=P_{m_{0}} \zeta+\xi$, where $z, \zeta \in N(L)$ and $y, \xi \in R(L)$. Let

$$
V_{0}(x):=\omega(\|\xi\|) V_{1}(\xi)+\omega(\|\zeta\|) P_{m_{0}} \widetilde{V}(\zeta) \quad \text { and } \quad V(x):=\frac{V_{0}(x)}{1+\left\|V_{0}(x)\right\|},
$$

where $\omega: \mathbf{R} \rightarrow[0,1]$ is a Lipschitz continuous function such that $\omega(s)=0$ for $s \leq \varepsilon / 2, \omega(s)=1$ for $s \geq \varepsilon$ and $\varepsilon>0$ is so small that $\bar{B}(0, \varepsilon) \cap R(L) \subset \operatorname{int}\left(W_{1}\right)$, $\bar{B}(0, \varepsilon) \cap N(L) \subset \operatorname{int}(\widetilde{W})$. According to Remark 2.4, it suffices to show that $V$ is an admissible field in a neighbourhood of $\partial W$. Note that without the cutoff function $\omega, V$ would not be defined on the subspaces $\xi=0$ and $\zeta=0$ (because respectively $V_{1}$ and $\widetilde{V}$ are not). Let $\eta_{1}, \widetilde{\eta}$ and $\eta$ be the flows of $-V_{1},-\widetilde{V}$ and $-V$. Then $\eta(t, x)=\eta_{1}\left(t_{1}, \xi\right)+P_{m_{0}} \widetilde{\eta}(\widetilde{t}, \zeta)$, and it is easy to see that condition (iv) of Definition 2.3 is satisfied. Since $\xi \in R(L) \cap E_{n}$ whenever $x \in E_{n}$ and $n \geq m_{0}, V$ is related to $\mathcal{E}$. It remains to show that $V$ is gradient-like in some neighbourhood $N$ of $\partial W$. Since $W$ is bounded, we may assume that $N$ is bounded. Then $\|\xi-y\|=\left\|P_{m_{0}} \zeta-z\right\| \rightarrow 0$ uniformly in $x \in N$ as $m_{0} \rightarrow \infty$, and therefore

$$
\begin{aligned}
\left\langle V_{0}(x), L y+\nabla \widetilde{\varphi}(z)\right\rangle= & \omega(\|\xi\|)\left\langle V_{1}(\xi), L y\right\rangle+\omega(\|\zeta\|)\langle\widetilde{V}(\zeta), \nabla \widetilde{\varphi}(z)\rangle \\
& +\omega(\|\zeta\|)\left\langle\left(P_{m_{0}}-I\right) \widetilde{V}(\zeta), L y+\nabla \widetilde{\varphi}(z)\right\rangle \\
\geq & \omega(\|\xi\|) \beta_{1}(\xi)+\omega(\|\zeta\|) \widetilde{\beta}(\zeta)-\varepsilon_{m_{0}},
\end{aligned}
$$

where $\varepsilon_{m_{0}} \rightarrow 0$ as $m_{0} \rightarrow \infty$ and $\beta_{1}, \widetilde{\beta}$ are as in Definition 2.1. We may assume the neighbourhood $N$ has been chosen in such a way that $x \notin N$ if $\|\xi\|<\varepsilon$ and $\|\zeta\|<\varepsilon$. Taking $m_{0}$ large enough, we see that $\langle V(x), L y+\nabla \widetilde{\varphi}(z)\rangle$ is positive and bounded away from 0 on $N$. Hence $\left(W, W^{-}\right)$is an admissible pair.

If $n \geq m_{0}$, then $\left(W_{1}+W_{2}\right) \cap E_{n}=\left(W_{1} \cap E_{n}\right)+W_{2}$. We need to compute the cohomology of

$$
\left(\left(W_{1} \cap E_{n}\right)+W_{2},\left(\left(W_{1}^{-} \cap E_{n}\right)+W_{2}\right) \cup\left(\left(W_{1} \cap E_{n}\right)+W_{2}^{-}\right)\right) .
$$

Topologically this pair is equivalent to

$$
\left(W_{1} \cap E_{n}, W_{1}^{-} \cap E_{n}\right) \times\left(W_{2}, W_{2}^{-}\right),
$$

where we have used the customary notation

$$
\left(A, A_{0}\right) \times\left(B, B_{0}\right)=\left(A \times B,\left(A \times B_{0}\right) \cup\left(A_{0} \times B\right)\right) .
$$

Let $B$ be a closed ball of dimension $m_{n}:=M^{-}\left(\left.Q_{n} L\right|_{R(L) \cap E_{n}}\right)$. For almost all $n$, $\left(W_{1} \cap E_{n}, W_{1}^{-} \cap E_{n}\right)$ is homotopy equivalent to $(B, \partial B)$ (cf. the proof of Theorem 
5.3). It follows from the Künneth formula [17, Proposition VI.12.16], [39, Theorem 5.6.1] that

$$
\begin{gathered}
H^{q+d_{n}}\left(\left(W_{1} \cap E_{n}, W_{1}^{-} \cap E_{n}\right) \times\left(W_{2}, W_{2}^{-}\right)\right) \cong H^{q+d_{n}}\left((B, \partial B) \times\left(W_{2}, W_{2}^{-}\right)\right) \\
\cong\left[H^{*}(B, \partial B) \otimes H^{*}\left(W_{2}, W_{2}^{-}\right)\right]^{q+d_{n}} \cong H^{q+d_{n}-m_{n}}\left(W_{2}, W_{2}^{-}\right)
\end{gathered}
$$

(note that the hypotheses in the Künneth formula are satisfied because the Čech cohomology has the strong excision property and $(B, \partial B)$ is a pair of ANRs). If $M_{\mathcal{E}}^{-}(L)$ is finite, then $q+d_{n}-m_{n}=q-M_{\mathcal{E}}^{-}(L)$ for almost all $n$, and the first conclusion follows. If $M_{\mathcal{E}}^{-}(L)=+\infty$ or $-\infty$, then respectively $q+d_{n}-m_{n}<0$ or $q+d_{n}-m_{n}>\operatorname{dim} N(L)$ for almost all $n$, so the right-hand side above is 0 for such $n$. This completes the proof.

Corollary 5.5. Suppose that $\Phi$ satisfies the hypotheses of Theorem 5.4 and $M_{\mathcal{E}}^{-}(L)$ is finite. If $\varphi(x) \geq \varphi(p)=0$ for all $x \in U$ ( $\varphi$ is given by the formula (5.6)), then $c_{\mathcal{E}}^{q}(\Phi, p)=[\mathcal{F}]$ for $q=M_{\mathcal{E}}^{-}(L)$ and $[0]$ otherwise. If $\varphi(x) \leq 0$ for all $x \in U$, then $c_{\mathcal{E}}^{q}(\Phi, p)=[\mathcal{F}]$ for $q=M_{\mathcal{E}}^{-}(L)+M^{0}(L)$ and $[0]$ otherwise.

Proof. Let $R(L)=E^{+} \oplus E^{-}$be the decomposition corresponding to the positive and the negative part of the spectrum of $L$. There exists a constant $c>0$ such that $\langle L y, y\rangle \geq c\|y\|^{2}$ for $y \in E^{+}$and $\langle L y, y\rangle \leq-c\|y\|^{2}$ for $y \in E^{-}$. According to (5.8) (with $\left.\alpha(z)=\alpha^{+}(z)+\alpha^{-}(z) \in E^{+} \oplus E^{-}\right)$,

$$
\begin{aligned}
\widetilde{\varphi}(z) & =\frac{1}{2}\left\langle L \alpha^{+}(z), \alpha^{+}(z)\right\rangle+\varphi\left(p+z+\alpha^{+}(z)\right) \\
& +\frac{1}{2}\left\langle L \alpha^{-}(z), \alpha^{-}(z)\right\rangle+\left(\varphi(p+z+\alpha(z))-\varphi\left(p+z+\alpha^{+}(z)\right)\right)
\end{aligned}
$$

for all $z \in B(0, \delta) \cap N(L)$. We claim that $\widetilde{\varphi}$ has a local minimum at 0 if $\varphi(x) \geq 0$ for all $x \in U$. Since

$$
\widetilde{\varphi}(z) \geq \frac{1}{2}\left\langle L \alpha^{-}(z), \alpha^{-}(z)\right\rangle+\left(\varphi(p+z+\alpha(z))-\varphi\left(p+z+\alpha^{+}(z)\right)\right),
$$

it suffices to show that the right-hand side above is nonnegative.

Let $\sigma:[0,1] \rightarrow E$ be given by $\sigma(t):=p+z+\alpha^{+}(z)+t \alpha^{-}(z)$. Then

$$
\begin{aligned}
\varphi & (p+z+\alpha(z))-\varphi\left(p+z+\alpha^{+}(z)\right)=\int_{0}^{1} \frac{d}{d t} \varphi(\sigma(t)) d t=\int_{0}^{1}\left\langle\nabla \varphi(\sigma(t)), \alpha^{-}(z)\right\rangle d t \\
& =\int_{0}^{1}\left\langle\nabla \varphi(\sigma(t))-\nabla \varphi(p+z+\alpha(z)), \alpha^{-}(z)\right\rangle d t+\left\langle\nabla \varphi(p+z+\alpha(z)), \alpha^{-}(z)\right\rangle \\
& =\int_{0}^{1}\left\langle\nabla \varphi(\sigma(t))-\nabla \varphi(p+z+\alpha(z)), \alpha^{-}(z)\right\rangle d t-\left\langle L \alpha^{-}(z), \alpha^{-}(z)\right\rangle,
\end{aligned}
$$

where the last equality follows from (5.7). Moreover, since $\varphi^{\prime \prime}(p)=0$, we may assume after choosing a smaller $\delta$ if necessary that

$$
\|\nabla \varphi(\sigma(t))-\nabla \varphi(p+z+\alpha(z))\| \leq c\|\sigma(t)-p-z-\alpha(z)\|=(1-t) c\left\|\alpha^{-}(z)\right\|
$$

for all $z \in B(0, \delta) \cap N(L)$. Hence

$$
\begin{aligned}
\widetilde{\varphi}(z) & \geq \frac{1}{2}\left\langle L \alpha^{-}(z), \alpha^{-}(z)\right\rangle+\left(\varphi(p+z+\alpha(z))-\varphi\left(p+z+\alpha^{+}(z)\right)\right) \\
& \geq-\frac{1}{2}\left\langle L \alpha^{-}(z), \alpha^{-}(z)\right\rangle-\int_{0}^{1}(1-t) c\left\|\alpha^{-}(z)\right\|^{2} d t \geq 0
\end{aligned}
$$


which proves the claim. So $c^{q}(\widetilde{\varphi}, 0)=\mathcal{F}$ if $q=0$ and 0 otherwise (see [15] or [34, Corollary 8.4]). It follows from Theorem 5.4 that $c_{\mathcal{E}}^{q}(\Phi, p) \cong\left[c^{q-M_{\mathcal{E}}^{-}(L)}(\widetilde{\varphi}, 0)\right]=[\mathcal{F}]$ for $q=M_{\mathcal{E}}^{-}(L)$ and $[0]$ otherwise.

If $\varphi(x) \leq 0$ for all $x \in U$, a similar argument shows that $\widetilde{\varphi}$ has a local maximum at 0 . Hence employing Theorem 5.4 and $[15,34]$ we obtain $c_{\mathcal{E}}^{q}(\Phi, p) \cong$ $\left[c^{q-M_{\mathcal{E}}^{-}(L)}(\widetilde{\varphi}, 0)\right]=[\mathcal{F}]$ for $q=M_{\mathcal{E}}^{-}(L)+M^{0}(L)$ and $[0]$ otherwise.

A functional $\Phi$ is said to satisfy the local linking condition at 0 if there exist a decomposition $E=Y \oplus Z$ and a constant $\rho>0$ such that

$$
\Phi(y) \leq c:=\Phi(0) \quad \text { for each } y \in B(0, \rho) \cap Y
$$

and

$$
\Phi(z) \geq c \quad \text { for each } z \in B(0, \rho) \cap Z .
$$

This is a variant of a condition introduced in [25].

Clearly, the functional $\Phi$ in Corollary 5.5 satisfies the local linking condition at $p$. If $\Phi \notin C^{2}(E, \mathbf{R})$, the argument of this corollary can no longer be applied. However, we still have the following weaker result:

Theorem 5.6. Suppose that $\Phi \in C^{1}(E, \mathbf{R})$ satisfies $(P S)^{*}$ and the local linking condition at 0 (with $Y$ and $Z$ as above). If 0 is an isolated critical point of $\Phi$, $E_{n}=\left(Y \cap E_{n}\right) \oplus\left(Z \cap E_{n}\right)$ and $\operatorname{dim}\left(Y \cap E_{n}\right)=q_{0}+d_{n}$ for almost all $n$, then $c_{\mathcal{E}}^{q_{0}}(\Phi, 0) \neq[0]$.

Proof. We may assume that $\Phi(0)=0,0$ is the only critical point of $\Phi$ in $B(0, \rho)$ and $\sup _{B(0, \rho)}|\Phi|<\infty$. Let $\delta \in(0, \rho)$ and let $\left(W, W^{-}\right), W \subset B(0, \delta)$, be an admissible pair having the additional properties given in Proposition 2.6. Define

$$
A:=\left\{\eta(t, x) \in W: t \geq 0, x \in S\left(0, \delta_{1}\right) \cap Y\right\} .
$$

Since $\Phi \leq 0$ on $S\left(0, \delta_{1}\right) \cap Y$, for each $x \in A$ there is a unique $t(x)$ such that $\eta(t(x), x) \in W^{-}$. According to (iv) of Definition 2.3, $t(x)$ depends continuously on $x$. Hence the mapping

$$
\alpha(\lambda, x):= \begin{cases}\eta(\lambda t(x), x) & \text { if } x \in A, 0 \leq \lambda \leq 1 \\ x & \text { if } x \in W^{-}, 0 \leq \lambda \leq 1,\end{cases}
$$

is a filtration-preserving strong deformation retraction of $A \cup W^{-}$onto $W^{-}$. So it follows from the exact sequence of the triple $\left(W, A \cup W^{-}, W^{-}\right)$that

$$
H_{\mathcal{E}}^{*}\left(W, W^{-}\right) \cong H_{\mathcal{E}}^{*}\left(W, A \cup W^{-}\right) .
$$

For $z \in Z$, let $\delta_{1}(z):=\min \left\{\delta_{1}, d\left(z, A \cup W^{-}\right)\right\}$and

$$
D:=\left\{y+z \in Y \oplus Z:\|y\|<\delta_{1}(z)\right\} .
$$

Since $\delta_{1}(z)>0 \forall z \in Z$ and $\left.\Phi\right|_{W^{-}}<0,\left(A \cup W^{-}\right) \cap Z=\emptyset$. Hence $D$ is an open set, $Z \subset D$ and $\left(A \cup W^{-}\right) \cap D=\emptyset$. Denote $F_{\delta}:=(\bar{B}(0, \delta) \cap Y) \oplus(\bar{B}(0, \delta) \cap Z)$ and let $i:\left(\bar{B}\left(0, \delta_{1}\right) \cap Y, S\left(0, \delta_{1}\right) \cap Y\right) \rightarrow\left(W, A \cup W^{-}\right), j:\left(W, A \cup W^{-}\right) \rightarrow\left(F_{\delta}, F_{\delta}-D\right)$ be the inclusion mappings. Then we have

$$
H_{\mathcal{E}}^{*}\left(F_{\delta}, F_{\delta}-D\right) \stackrel{j^{*}}{\longrightarrow} H_{\mathcal{E}}^{*}\left(W, A \cup W^{-}\right) \stackrel{i^{*}}{\longrightarrow} H_{\mathcal{E}}^{*}\left(\bar{B}\left(0, \delta_{1}\right) \cap Y, S\left(0, \delta_{1}\right) \cap Y\right),
$$


where $i^{*}, j^{*}$ are the induced homomorphisms. It is easy to see that the mapping

$$
\gamma(\lambda, y+z):= \begin{cases}\frac{2 \lambda \delta_{1} y}{\max \left\{\|y\|, \delta_{1}(z)\right\}}+(1-2 \lambda) y+z, & 0 \leq \lambda \leq \frac{1}{2}, \\ \frac{\delta_{1} y}{\max \left\{\|y\|, \delta_{1}(z)\right\}}+(2-2 \lambda) z, & \frac{1}{2} \leq \lambda \leq 1\end{cases}
$$

is a deformation of $\left(F_{\delta}, F_{\delta}-D\right)$ onto $\left(\bar{B}\left(0, \delta_{1}\right) \cap Y, S\left(0, \delta_{1}\right) \cap Y\right)$. It preserves the filtration since $y, z \in E_{n}$ whenever $y+z \in E_{n}$. Moreover, the restriction of $\gamma$ to $[0,1] \times\left(\bar{B}\left(0, \delta_{1}\right) \cap Y, S\left(0, \delta_{1}\right) \cap Y\right)$ is a homotopy between $\gamma(1,.) \circ(j i)$ and the identity on $\left(\bar{B}\left(0, \delta_{1}\right) \cap Y, S\left(0, \delta_{1}\right) \cap Y\right)$. Similarly, $\gamma$ is a homotopy between $(j i) \circ \gamma(1,$.$) and the identity on \left(F_{\delta}, F_{\delta}-D\right)$. So the inclusion mapping $j i$ is a homotopy equivalence by filtration-preserving homotopies, and it follows that $i^{*} j^{*}$ in (5.13) is an isomorphism. In particular, $H_{\mathcal{E}}^{q_{0}}\left(W, W^{-}\right) \cong H_{\mathcal{E}}^{q_{0}}\left(W, A \cup W^{-}\right)$ is nontrivial because $H_{\mathcal{E}}^{q_{0}}\left(\bar{B}\left(0, \delta_{1}\right) \cap Y, S\left(0, \delta_{1}\right) \cap Y\right)=[\mathcal{F}]$ according to Example 1.4 .

\section{Relation to Degree theory}

Assume that $U \subset E$ is an open neighbourhood of an isolated critical point $p$ of $\Phi \in C^{2}(U, \mathbf{R}), \nabla \Phi(x)=x-T(x)$ and $T$ is compact. Define the Leray-Schauder index of $\nabla \Phi$ at $p$ as

$$
\operatorname{ind}(\nabla \Phi, p):=\operatorname{deg}(I-T, B(p, \rho), 0),
$$

where $B(p, \rho) \subset U$ and on the right-hand side we have the Leray-Schauder degree of $I-T$ with respect to $B(p, \rho)$ and 0 (see e.g. [31]). According to [10, Theorem II.3.2], cf. also [34, Theorem 8.5],

$$
\operatorname{ind}(\nabla \Phi, p)=\sum_{q=0}^{\infty}(-1)^{q} \operatorname{dim} c_{q}(\Phi, p)
$$

$\left(c_{q}\right.$ are the critical groups defined in (0.1)). It follows by inspection of the proof in [10] that (6.1) remains valid with $c_{q}(\Phi, p)$ replaced by the critical Cech cohomology groups $c^{q}(\Phi, p)$ of $\Phi$ at $p$ (with respect to the trivial filtration of $E$; cf. Remark $3.4)$. Recall that the Euler characteristic for a pair $(X, B)$ of finite type is defined by

$$
\chi(X, B)=\sum_{q=0}^{\infty}(-1)^{q} \operatorname{dim} H^{q}(X, B) \equiv P(-1, X, B),
$$

where $P(t, X, B)$ is the Poincaré polynomial of $(X, B)$.

Let $\left(W, W^{-}\right)$be a bounded admissible pair for $\Phi$ and $p$ (again, with respect to the trivial filtration of $E)$. Since $c^{*}(\Phi, p)=H^{*}\left(W, W^{-}\right),(6.1)$ may be reformulated as

$$
\operatorname{deg}(\nabla \Phi, \operatorname{int}(W), 0)=\chi\left(W, W^{-}\right),
$$

and this relation remains valid also if $\left(W, W^{-}\right)$is admissible for $\Phi$ and a set $A \subset K$, cf. [10, Theorem II.3.3]. Formula (6.2) may be seen as a generalization of the Poincaré-Hopf theorem.

Recall from [31] that a mapping $T: X \rightarrow Y$ is said to be a $k$-set contraction if for each bounded set $B \subset X, \alpha(T(B)) \leq k \alpha(B)$, where $\alpha$ is the (Kuratowski) measure of noncompactness. $T$ is called a strict set contraction if it is a $k$-set contraction with $k<1$. For mappings $f$ of the form $f(x)=x-T(x)$, where $T$ is 
a strict set contraction, there exists a degree theory (see [31, Section 6.2]) which coincides with the Leray-Schauder degree if $T$ is compact (compact mappings are 0 -set contractions). One can verify that formula (6.2) remains valid if $\nabla \Phi(x)=$ $x-T(x)$ and $T$ is a strict set contraction (this will be done in the course of the proof of Theorem 6.1).

In this section we will look for a possible generalization of formula (6.2) to the case of strongly indefinite functionals.

Let $\left(E_{n}\right)_{n=1}^{\infty}$ be a filtration of $E,\left(d_{n}\right)_{n=1}^{\infty}$ a sequence of nonnegative integers and $\mathcal{E}:=\left\{E_{n}, d_{n}\right\}_{n=1}^{\infty}$. Suppose that $\Phi \in C^{1}(E, \mathbf{R}), U$ is an open bounded subset of $E$ and

$$
\begin{aligned}
& \Phi \text { satisfies }(P S)^{*} \text { on } \bar{U}, \\
& \left.\Phi\right|_{U} \text { is bounded, } \\
& \Phi \text { has no critical points on } \partial U, \\
& P_{n} \nabla \Phi(x)=x-T_{n}(x) \text { for all } n \geq 1 \text { and } x \in \overline{U \cap E_{n}} \text {, where } \\
& T_{n}: \overline{U \cap E_{n}} \rightarrow E_{n} \text { is a strict set contraction. }
\end{aligned}
$$

Clearly, (6.6) is satisfied if all $E_{n}$ are finite dimensional or all $T_{n}$ are compact. Denote $U_{n}:=U \cap E_{n}$ and $\Phi_{n}:=\left.\Phi\right|_{E_{n}}$. It is easy to see that $U_{n}$ is nonempty for almost all $n, \bar{U}_{n} \subset \bar{U} \cap E_{n}, \partial U_{n} \subset \partial U \cap E_{n}$ and $\nabla \Phi_{n}=\left.P_{n} \nabla \Phi\right|_{E_{n}}$. We are going to define a generalized topological degree of $\nabla \Phi$ with respect to $U$ and 0 . Observe that for large $n$, say $n \geq n_{0}, 0 \notin \nabla \Phi_{n}\left(\partial U_{n}\right)$. Indeed, otherwise for each $j$ there are $n_{j} \geq j$ and $y_{j} \in \partial U_{n_{j}}$ such that $\nabla \Phi_{n_{j}}\left(y_{j}\right)=0$. By $(\mathrm{PS})^{*}$ (recall $\left.\Phi\right|_{U}$ is bounded), $\left(y_{j}\right)$ has a subsequence converging to some $y \in K \cap \partial U$, a contradiction to (6.5). Therefore

$$
s_{n}:=\operatorname{deg}\left(\nabla \Phi_{n}, U_{n}, 0\right)
$$

is well-defined for $n \geq n_{0}$. For $n<n_{0}$ we put $s_{n}:=0$. Now we define the $\mathcal{E}$-degree of $\nabla \Phi$ with respect to $U$ and 0 by the formula

$$
\operatorname{Deg}_{\mathcal{E}}(\nabla \Phi, U, 0):=\left[\left((-1)^{d_{n}} s_{n}\right)_{n=1}^{\infty}\right] .
$$

Note that $\operatorname{Deg}_{\mathcal{E}}(\nabla \Phi, U, 0) \in[\mathbf{Z}]$. The above definition is modelled on the definition of the generalized degree for A-proper mappings given in [24] (in [24] there are no terms $\left.(-1)^{d_{n}}\right)$.

It is easy to see that $\operatorname{Deg}_{\mathcal{E}}$ satisfies the usual properties of topological degree except that $\operatorname{Deg}_{\mathcal{E}}(I, U, 0)=\left[\left((-1)^{d_{n}}\right)_{n=1}^{\infty}\right]$ (instead of being equal to $\left[(1)_{n=1}^{\infty}\right]$ ) if $0 \in U$. Admissible homotopies are the ones which preserve the filtration and map $\partial U_{n} \times[0,1]$ into $E_{n}-\{0\}$ for almost all $n$. By the excision property, it is possible to define the $\mathcal{E}$-index of $\nabla \Phi$ at an isolated critical point $p \in U$ by setting

$$
\operatorname{Ind}_{\mathcal{E}}(\nabla \Phi, p):=\operatorname{Deg}_{\mathcal{E}}(\nabla \Phi, B(p, \rho), 0),
$$

where $\rho$ is such that $B(p, \rho) \subset U$ and $\bar{B}(p, \rho) \cap K=\{p\}$.

Define the $\mathcal{E}$-Euler characteristic of an $\mathcal{E}$-finite pair $(X, B)$ of closed subsets of E by

$$
\chi_{\mathcal{E}}(X, B):=P_{\mathcal{E}}(-1, X, B) \equiv \sum_{q=-\infty}^{\infty}(-1)^{q} \operatorname{dim}_{\mathcal{E}} H_{\mathcal{E}}^{q}(X, B) .
$$

The pair $(X, B)$ will be called strongly $\mathcal{E}$-finite if there is a $k \geq 1$ such that $\operatorname{dim} H^{q+d_{n}}\left(X \cap E_{n}, B \cap E_{n}\right)$ is finite for all $q \in \mathbf{Z}$ and $n \geq k$, and zero for all $|q| \geq k$ and $n \geq k$. Similarly, an isolated critical point $p$ of a functional $\Phi$ which 
satisfies $(\mathrm{PS})^{*}$ will be called strongly $\mathcal{E}$-finite if so is some (and therefore every) admissible pair for $\Phi$ and $p$. Note that strongly $\mathcal{E}$-finite implies $\mathcal{E}$-finite (but not conversely, as will be seen from Remark 6.3 below). The reason for introducing the notion of strong $\mathcal{E}$-finiteness is that then

$$
\chi_{\mathcal{E}}(X, B)=\left[\left((-1)^{d_{n}} \chi\left(X \cap E_{n}, B \cap E_{n}\right)\right)_{n=1}^{\infty}\right],
$$

and in general this equality is not true for $\mathcal{E}$-finite pairs (again, Remark 6.3 will provide an example). To show (6.7), observe that if $n \geq k$, then

$$
\begin{aligned}
(-1)^{d_{n}} \chi\left(X \cap E_{n}, B \cap E_{n}\right) & =(-1)^{d_{n}} \sum_{q=0}^{\infty}(-1)^{q} \operatorname{dim} H^{q}\left(X \cap E_{n}, B \cap E_{n}\right) \\
& =\sum_{q=-\infty}^{\infty}(-1)^{q} \operatorname{dim} H^{q+d_{n}}\left(X \cap E_{n}, B \cap E_{n}\right) .
\end{aligned}
$$

Since all terms on the right-hand side are zero for $|q| \geq k$, the conclusion follows from the definition of $\chi_{\mathcal{E}}$.

Now we state the main result of this section.

Theorem 6.1. Suppose that $\Phi \in C^{2}(E, \mathbf{R})$ satisfies $(P S)^{*},\left(W, W^{-}\right)$is a bounded admissible pair for $\Phi$ and $A$, and $\nabla \Phi$ has the form (6.6) on $W$. Then

(i) $\operatorname{Deg}_{\mathcal{E}}(\nabla \Phi, \operatorname{int}(W), 0)=\left[\left((-1)^{d_{n}} \chi\left(W \cap E_{n}, W^{-} \cap E_{n}\right)\right)_{n=1}^{\infty}\right]$;

(ii) If $\left(W, W^{-}\right)$is strongly $\mathcal{E}$-finite, then $\operatorname{Deg}_{\mathcal{E}}(\nabla \Phi, \operatorname{int}(W), 0)=\chi_{\mathcal{E}}\left(W, W^{-}\right)$; then

(iii) (Poincaré-Hopf formula) If $A=\left\{p_{1}, \ldots, p_{k}\right\}$ and all $p_{j}$ are strongly $\mathcal{E}$-finite,

$$
\sum_{j=1}^{k} \operatorname{Ind}_{\mathcal{E}}\left(\nabla \Phi, p_{j}\right)=\chi_{\mathcal{E}}\left(W, W^{-}\right) .
$$

Proof. (i) Since (6.3)-(6.6) are satisfied (with $U=\operatorname{int}(W)), \operatorname{Deg}_{\mathcal{E}}(\nabla \Phi, \operatorname{int}(W), 0)$ is well-defined. It is easy to see that for almost all $n,\left(W_{n}, W_{n}^{-}\right):=\left(W \cap E_{n}, W^{-} \cap E_{n}\right)$ is an admissible pair for $\Phi_{n}$ and $A_{n}:=W \cap K\left(\Phi_{n}\right)$ (with respect to the trivial filtration of $\left.E_{n}\right)$. Indeed, if $V$ is an admissible field for $\left(W, W^{-}\right)$and $N$ is a sufficiently small neighbourhood of $\partial W$, then $V$ maps $N \cap E_{n}$ into $E_{n}$ for all large $n$. According to Remark 2.4, this suffices for $\left(W_{n}, W_{n}^{-}\right)$to be an admissible pair. Since $\left.\nabla \Phi_{n}\right|_{W_{n}}$ satisfies (6.6), it is a proper mapping; cf. [31, Corollary 6.2.2]. Therefore $\Phi_{n}$ satisfies (PS) on $W_{n}$. In particular, the set $A_{n}$ is compact. Now it follows essentially from [10, Theorem II.3.3] that

$$
\operatorname{deg}\left(\nabla \Phi_{n}, \operatorname{int}(W) \cap E_{n}, 0\right)=\chi\left(W_{n}, W_{n}^{-}\right),
$$

and (i) is satisfied in view of the definition of $\operatorname{Deg}_{\mathcal{E}}$.

For the reader's convenience and since the hypotheses in [10] are somewhat different from ours, we give a proof of (6.8). Since $A_{n}$ is compact, we may find a $\delta>0$ and a function $\varepsilon \in C^{2}\left(E_{n},[0,1]\right)$ such that $\varepsilon(x)=1$ if $x \in G:=\{x \in$ $\left.E_{n}: d\left(x, A_{n}\right) \leq \delta\right\}$ and $\varepsilon(x)=0$ if $d\left(x, \partial W_{n}\right) \leq \delta$. Moreover, we may assume that $\|\nabla \varepsilon(x)\| \leq m$ and $\left\|\varepsilon^{\prime \prime}(x)\right\| \leq m$ for some constant $m$ and all $x$. Let $M:=\sup \{\|x\|:$ $\left.x \in W_{n}\right\}$ and

$$
\beta:=\inf _{x \in W_{n}-G}\left\|\nabla \Phi_{n}(x)\right\| .
$$

Clearly, $\beta>0$. It follows from a version of the Sard-Smale theorem for $k$-set contractions [44] that we can find an arbitrarily small $x_{0} \in E_{n}$ such that $\left(\nabla \Phi_{n}\right)^{-1}\left(x_{0}\right) \cap$ 
$W_{n}=\left\{p_{1}, \ldots, p_{s}\right\} \subset G$ and $\Phi_{n}^{\prime \prime}\left(p_{j}\right)$ is invertible for all $j$. Let

$$
\psi_{n}(x):=\Phi_{n}(x)+\varepsilon(x)\left\langle x, x_{0}\right\rangle .
$$

For $x \in W_{n}-G$ we have

$$
\left\|\nabla \psi_{n}(x)\right\| \geq\left\|\nabla \Phi_{n}(x)\right\|-\left\|x_{0}\right\|-\|\nabla \varepsilon(x)\|\|x\|\left\|x_{0}\right\| \geq \frac{\beta}{2}
$$

provided $\left\|x_{0}\right\|$ is small enough. Hence $K\left(\psi_{n}\right) \cap W_{n}=\left\{p_{1}, \ldots, p_{s}\right\}$. Since $\psi_{n}(x)=$ $\Phi_{n}(x)$ if $d\left(x, \partial W_{n}\right) \leq \delta,\left(W_{n}, W_{n}^{-}\right)$is an admissible pair for $\psi_{n}$ and $\left\{p_{1}, \ldots, p_{s}\right\}$. Now observe that for $x \in W_{n}$,

$$
\nabla \psi_{n}(x)=x-T_{n}(x)+\left\langle x, x_{0}\right\rangle \nabla \varepsilon(x)+\varepsilon(x) x_{0},
$$

where $T_{n}$ is a $k$-set contraction. Since $x \mapsto\left\langle x, x_{0}\right\rangle \nabla \varepsilon(x)+\varepsilon(x) x_{0}$ is a Lipschitz continuous mapping with Lipschitz constant $(M+2) m\left\|x_{0}\right\|$ which will be less than $1-k$ if we choose a sufficiently small $x_{0}$, we obtain that $x \mapsto T_{n}(x)-\left\langle x, x_{0}\right\rangle \nabla \varepsilon(x)-\varepsilon(x) x_{0}$ is a strict set contraction [31, Theorems 6.1.8 and 6.1.9]. Hence $\operatorname{deg}\left(\nabla \psi_{n}, U_{n}, 0\right)$, where $U_{n}:=\operatorname{int}(W) \cap E_{n}$, is well-defined. Since $\psi_{n}=\Phi_{n}$ on $\partial U_{n}$,

$$
\operatorname{deg}\left(\nabla \Phi_{n}, U_{n}, 0\right)=\operatorname{deg}\left(\nabla \psi_{n}, U_{n}, 0\right) .
$$

We will apply Theorem 3.1 to $\psi_{n},\left(W_{n}, W_{n}^{-}\right)$and $\left\{p_{1}, \ldots, p_{s}\right\}$. Since each $p_{j}$ is nondegenerate,

$$
\operatorname{ind}\left(\nabla \psi_{n}, p_{j}\right)=(-1)^{k_{j}}=\sum_{q=0}^{\infty}(-1)^{q} \operatorname{dim} c^{q}\left(\psi_{n}, p_{j}\right),
$$

where $k_{j}$ is the Morse index of $\psi_{n}^{\prime \prime}\left(p_{j}\right)$. For the first equality, see [31, Theorem 8.1.1 and Remark on p. 122]. The second equality is an easy consequence of Theorem 5.3 (recall that $c^{q}$ are the critical groups with respect to the trivial filtration of $E_{n}$ ). By the additivity property of degree,

$$
\begin{aligned}
\operatorname{deg}\left(\nabla \psi_{n}, U_{n}, 0\right) & =\sum_{j=1}^{s} \operatorname{ind}\left(\nabla \psi_{n}, p_{j}\right)=\sum_{q=0}^{\infty}(-1)^{q} \sum_{j=1}^{s} \operatorname{dim} c^{q}\left(\psi_{n}, p_{j}\right) \\
& =M\left(-1, W_{n}, W_{n}^{-}\right)
\end{aligned}
$$

( $M$ is the Morse polynomial). By Theorem 3.1, $M\left(-1, W_{n}, W_{n}^{-}\right)=P\left(-1, W_{n}, W_{n}^{-}\right)$ $\equiv \chi\left(W_{n}, W_{n}^{-}\right)$, and (6.8) follows from (6.9).

(ii) This is a direct consequence of (6.7) and (i).

(iii) Let $\left(W_{j}, W_{j}^{-}\right)$be an admissible pair for $\Phi$ and $p_{j}$. We may assume that $W_{j} \subset W$ for all $j$ and $W_{i} \cap W_{j}=\emptyset$ if $i \neq j$. By the definition of $M_{\mathcal{E}}$,

$$
M_{\mathcal{E}}\left(t, W, W^{-}\right)=\sum_{j=1}^{k} M_{\mathcal{E}}\left(t, W_{j}, W_{j}^{-}\right) .
$$

Hence, according to (ii) and Theorem 3.1,

$$
\sum_{j=1}^{k} \operatorname{Ind}_{\mathcal{E}}\left(\nabla \Phi, p_{j}\right)=M_{\mathcal{E}}\left(-1, W, W^{-}\right)=P_{\mathcal{E}}\left(-1, W, W^{-}\right) \equiv \chi_{\mathcal{E}}\left(W, W^{-}\right) .
$$

Alternatively, one can show (cf. the proof of Theorem 3.1) that $\left(W, W^{-}\right)$is strongly $\mathcal{E}$-finite if all $p_{j}$ are, and then the conclusion follows from (ii) and the additivity property of degree. 
Remark 6.2. If all $E_{n}$ are finite dimensional, then (6.6) is trivially satisfied and it suffices to assume that $\Phi \in C^{1}(E, \mathbf{R})$. Indeed, $\left.\Phi_{n}\right|_{W_{n}}$ can be approximated in the $C^{1}$-topology by a $C^{2}$-function $\widetilde{\Phi}_{n}$ such that the degree on $U_{n}$ is unchanged and $\left(W_{n}, W_{n}^{-}\right)$is admissible for $\widetilde{\Phi}_{n}$ and $K\left(\widetilde{\Phi}_{n}\right) \cap W_{n}$.

Remark 6.3. If $\left(W, W^{-}\right)$is $\mathcal{E}$-finite, but not strongly, then (ii) and (iii) of Theorem 6.1 need not hold. To see this, let $\Phi$ and $p$ be as in Theorem 5.3, with $m_{n}:=$ $M^{-}\left(\left.P_{n} L\right|_{E_{n}}\right)<\infty$ and $M_{\mathcal{E}}^{-}(L)=+\infty$ or $-\infty$. If $\left(W, W^{-}\right)$is an admissible pair for $\Phi$ and $p$, then $H_{\mathcal{E}}^{q}\left(W, W^{-}\right)=[0]$ for all $q$. So $\chi_{\mathcal{E}}\left(W, W^{-}\right)=[0]$, while

$$
\operatorname{Ind}_{\mathcal{E}}(\nabla \Phi, p)=\operatorname{Deg}_{\mathcal{E}}(\nabla \Phi, \operatorname{int}(W), 0)=\left[\left((-1)^{m_{n}-d_{n}}\right)_{n=1}^{\infty}\right] \neq[0] .
$$

\section{Hamiltonian SYSTEMS}

Let

$$
J:=\left(\begin{array}{cc}
0 & -I \\
I & 0
\end{array}\right)
$$

be the standard symplectic matrix and consider the Hamiltonian system of differential equations

$$
\dot{z}=J H_{z}(z, t),
$$

where $H \in C^{1}\left(\mathbf{R}^{2 N} \times \mathbf{R}, \mathbf{R}\right)$ is $2 \pi$-periodic in $t$. In this section we study the existence of $2 \pi$-periodic solutions of (7.1). Let $E:=H^{1 / 2}\left(S^{1}, \mathbf{R}^{2 N}\right)$ be the Sobolev space of $2 \pi$-periodic $\mathbf{R}^{2 N}$-valued functions

$$
z(t)=a_{0}+\sum_{k=1}^{\infty} a_{k} \cos k t+b_{k} \sin k t, \quad a_{0}, a_{k}, b_{k} \in \mathbf{R}^{2 N},
$$

such that $\sum_{k=1}^{\infty} k\left(\left|a_{k}\right|^{2}+\left|b_{k}\right|^{2}\right)<\infty$. Then $E$ is a Hilbert space with inner product $\langle.,$.$\rangle defined by$

$$
\left\langle z, z^{\prime}\right\rangle:=2 \pi a_{0} \cdot a_{0}^{\prime}+\pi \sum_{k=1}^{\infty} k\left(a_{k} \cdot a_{k}^{\prime}+b_{k} \cdot b_{k}^{\prime}\right) .
$$

Assume that $H_{z}$ is asymptotically linear at infinity. Then in particular

$$
\left|H_{z}(z, t)\right| \leq C(1+|z|)
$$

for some $C>0$ and all $(z, t)$. It is well-known [38] that under this condition $z(t)$ is a $2 \pi$-periodic solution of (7.1) if and only if it is a critical point of the functional

$$
\Phi(z):=\frac{1}{2} \int_{0}^{2 \pi}(-J \dot{z} \cdot z) d t-\int_{0}^{2 \pi} H(z, t) d t=\frac{1}{2}\langle\widetilde{L} z, z\rangle-\psi(z) .
$$

Moreover, $\Phi \in C^{1}(E, \mathbf{R})$ and $\nabla \psi$ is a compact mapping. Sometimes we will make a stronger assumption that $H \in C^{2}\left(\mathbf{R}^{2 N} \times \mathbf{R}, \mathbf{R}\right)$ and

$$
\left\|H_{z z}(z, t)\right\| \leq C^{\prime}\left(1+|z|^{s}\right)
$$

for some $C^{\prime}>0, s \in(0, \infty)$ and all $(z, t)$. Then $\Phi \in C^{2}(E, \mathbf{R})[38]$.

Let

$$
F_{0}:=\mathbf{R}^{2 N}, \quad F_{k}:=\left\{a_{k} \cos k t+b_{k} \sin k t: a_{k}, b_{k} \in \mathbf{R}^{2 N}\right\}, \quad \text { where } k \geq 1,
$$

and

$$
E_{n}:=F_{0} \oplus \bigoplus_{k=1}^{n} F_{k} \equiv\left\{z \in E: z(t)=a_{0}+\sum_{k=1}^{n} a_{k} \cos k t+b_{k} \sin k t\right\} .
$$


Then $\left(E_{n}\right)_{n=1}^{\infty}$ is a filtration of $E$. Set

$$
d_{n}:=N(1+2 n) \quad \text { and } \quad \mathcal{E}:=\left\{E_{n}, d_{n}\right\}_{n=1}^{\infty} .
$$

Consider the linear Hamiltonian system

$$
\dot{z}=J A z
$$

where $A$ is a symmetric $2 N \times 2 N$ matrix (with constant entries $a_{i j}$ ). Let $B: E \rightarrow E$ be the linear operator defined by

$$
\langle B z, w\rangle:=\int_{0}^{2 \pi} A z \cdot w d t .
$$

Then $B$ is compact, and it is easy to see that

$$
B z=A a_{0}+\sum_{k=1}^{\infty} \frac{1}{k}\left(A a_{k} \cos k t+A b_{k} \sin k t\right) .
$$

Following [26], cf. also [1,2], we now proceed to define the index and the nullity of A. According to (7.2) and (7.4),

$$
(\widetilde{L}-B)(a \cos k t+b \sin k t)=\left(-J b-\frac{1}{k} A a\right) \cos k t+\left(J a-\frac{1}{k} A b\right) \sin k t
$$

Hence $\widetilde{L}-B$ maps $F_{k}$ into itself and $\left.(\widetilde{L}-B)\right|_{F_{k}}, k \geq 1$, corresponds to a linear operator on $\mathbf{R}^{4 N}$ given by the matrix

$$
T_{k}(A):=\left(\begin{array}{cc}
-\frac{1}{k} A & -J \\
J & -\frac{1}{k} A
\end{array}\right)
$$

Since $T_{k}$ is symmetric, it has only real eigenvalues. For $k$ large enough, $M^{-}\left(T_{k}(A)\right)$ $=M^{+}\left(T_{k}(A)\right)=2 N$ and $M^{0}\left(T_{k}(A)\right)=0$, cf. [2, Section 2]. As usual, $M^{-}$and $M^{0}$ are the Morse index and the nullity of a corresponding quadratic form, and $M^{+}\left(T_{k}(A)\right):=M^{-}\left(-T_{k}(A)\right)$. Hence the numbers

$$
\begin{aligned}
& i^{-}(A):=M^{-}(-A)-N+\sum_{k=1}^{\infty}\left(M^{-}\left(T_{k}(A)\right)-2 N\right) \\
& i^{+}(A):=M^{+}(-A)-N+\sum_{k=1}^{\infty}\left(M^{+}\left(T_{k}(A)\right)-2 N\right)
\end{aligned}
$$

and

$$
i^{0}(A):=M^{0}(-A)+\sum_{k=1}^{\infty} M^{0}\left(T_{k}(A)\right)
$$

are well-defined and finite. Moreover, $i^{-}(A)+i^{+}(A)+i^{0}(A)=0$. Note that the numbers $i^{ \pm}(A)$ differ by $N$ from those introduced in [26].

Let $L:=\widetilde{L}-B$ and $M_{\mathcal{E}}^{+}(L):=M_{\mathcal{E}}^{-}(-L)$.

Proposition 7.1. $i^{0}(A)=M^{0}(L), i^{-}(A)=M_{\mathcal{E}}^{-}(L)=\widetilde{M}_{\mathcal{E}}^{-}(L)$ and $i^{+}(A)=$ $M_{\mathcal{E}}^{+}(L)=\widetilde{M}_{\mathcal{E}}^{+}(L)$. 
Proof. Clearly, $M^{0}(L) \equiv \operatorname{dim} N(L)=i^{0}(A)$. Since $L$ maps each $F_{k}, k \geq 0$, into itself, $N(L) \subset E_{n}$ for some $n$ and $\left.P_{n} L\right|_{E_{n}}=\left.L\right|_{E_{n}}$. Therefore $M_{\mathcal{E}}^{-}(L)=\widetilde{M}_{\mathcal{E}}^{-}(L)$ according to Remark 5.1 and

$$
M^{-}\left(\left.P_{n} L\right|_{E_{n}}\right)-d_{n}=M^{-}(-A)-N+\sum_{k=1}^{n}\left(M^{-}\left(T_{k}(A)\right)-2 N\right) .
$$

Invoking (5.2) we see that $i^{-}(A)=M_{\mathcal{E}}^{-}(L)$. Similarly, $i^{+}(A)=M_{\mathcal{E}}^{+}(L)$.

Suppose now that $A(t)$ is a symmetric $2 N \times 2 N$-matrix with continuous $2 \pi$ periodic entries $a_{i j}(t)$. Then $i^{-}(A)$ and $i^{+}(A)$ are no longer defined. Since the operator $B$ given by the formula

$$
\langle B z, w\rangle:=\int_{0}^{2 \pi} A(t) z \cdot w d t
$$

is compact, it follows from Proposition 5.2 that $L:=\widetilde{L}-B$ is A-proper and $M_{\mathcal{E}}^{-}(L)$ is well-defined and finite. Furthermore, $M^{0}(L)$ is the number of linearly independent $2 \pi$-periodic solutions of the linear system

$$
\dot{z}=J A(t) z,
$$

and therefore $0 \leq M^{0}(L) \leq 2 N$. Denote

$$
j^{-}(A):=M_{\mathcal{E}}^{-}(L), \quad j^{+}(A):=M_{\mathcal{E}}^{+}(L) \quad \text { and } \quad j^{0}(A):=M^{0}(L) .
$$

Since $M^{-}\left(\left.Q_{n} L\right|_{R(L) \cap E_{n}}\right)+M^{+}\left(\left.Q_{n} L\right|_{R(L) \cap E_{n}}\right)+M^{0}(L)=\operatorname{dim} E_{n}=2 d_{n}$ for almost all $n$ (cf. Lemma 4.2 and the beginning of Section 5 ), $j^{-}(A)+j^{+}(A)+j^{0}(A)=$ 0 . Although we will only be concerned with the numbers $j^{ \pm}(A)$ and $j^{0}(A)$, the following remark is in order:

Remark 7.2. To each matrix $A(t)$ as above there corresponds a unique solution $\gamma(t)$ of the initial value problem $\dot{\gamma}=J A(t) \gamma, \gamma(0)=I$ (the fundamental solution), and $\gamma(t)$ is a path in the space of symplectic matrices. If $j^{0}(A)=0$, it is possible to introduce an equivalence relation for these paths and show that there exists a constant matrix $A_{1}$ such that the corresponding fundamental solution $\gamma_{1}(t)$ is equivalent to $\gamma(t)$. Now one can define the Maslov index of $\gamma$ by setting $j(\gamma):=$ $i^{-}\left(A_{1}\right)$. See $[13,33]$ for more details. To be more precise, the definition of $j(\gamma)$ in $[13,33]$ differs from ours. However, it follows from [33, Theorems 2.1, 3.1], cf. also [10, Theorems IV.1.1, IV.1.2] and [13, Theorem 1 and Lemma 2.4], that $j^{-}(A)=$ $j(\gamma)=j\left(\gamma_{1}\right)=j^{-}\left(A_{1}\right)$. Since $i^{-}\left(A_{1}\right)=j^{-}\left(A_{1}\right)$ according to Proposition 7.1, the two definitions of $j(\gamma)$ are equivalent. If $j^{0}(A) \neq 0$, one can still define a Maslovtype index $(j(\gamma), n(\gamma))$ as has been shown by Long [32]. Moreover, $j(\gamma)=j^{-}(A)$ and $n(\gamma)=j^{0}(A)[32$, Theorem 6].

In what follows we assume that there exist two symmetric $2 N \times 2 N$ matrices $A(t)$ and $A_{0}(t)$ with $2 \pi$-periodic entries such that

$$
\begin{aligned}
& H(z, t)=\frac{1}{2} A(t) z \cdot z+G(z, t), \\
& \quad \text { where } G_{z}(z, t)=o(|z|) \text { uniformly in } t \text { as }|z| \rightarrow \infty
\end{aligned}
$$

and

$$
\begin{aligned}
& H(z, t)=\frac{1}{2} A_{0}(t) z \cdot z+G_{0}(z, t), \\
& \quad \text { where }\left(G_{0}\right)_{z}(z, t)=o(|z|) \text { uniformly in } t \text { as }|z| \rightarrow 0 .
\end{aligned}
$$


We will use the notation

$$
\begin{aligned}
\Phi(z) & =\frac{1}{2} \int_{0}^{2 \pi}(-J \dot{z}-A(t) z) \cdot z d t-\int_{0}^{2 \pi} G(z, t) d t=: \frac{1}{2}\langle L z, z\rangle-\varphi(z) \\
& =\frac{1}{2} \int_{0}^{2 \pi}\left(-J \dot{z}-A_{0}(t) z\right) \cdot z d t-\int_{0}^{2 \pi} G_{0}(z, t) d t=: \frac{1}{2}\left\langle L_{0} z, z\right\rangle-\varphi_{0}(z) .
\end{aligned}
$$

It is well-known (cf. e.g. [26] or [38]) that $\nabla \varphi(z)=o(\|z\|)$ as $\|z\| \rightarrow \infty$ and $\nabla \varphi_{0}(z)=o(\|z\|)$ as $z \rightarrow 0$. Indeed, for each $\varepsilon>0$ there is a $C(\varepsilon)$ such that $\left|G_{z}(z, t)\right| \leq \varepsilon|z|+C(\varepsilon)$. Hence

$$
|\langle\nabla \varphi(z), y\rangle| \leq \int_{0}^{2 \pi}(\varepsilon|z|+C(\varepsilon))|y| d t \leq\left(\varepsilon\|z\|+C^{\prime}(\varepsilon)\right)\|y\|
$$

for all $y \in E$. Taking the supremum over $\|y\| \leq 1$, dividing by $\|z\|$ and letting $\|z\| \rightarrow \infty$, we see that $\nabla \varphi(z)=o(\|z\|)$. Similarly, for each $\varepsilon>0$ there is a $C(\varepsilon)$ such that $\left|\left(G_{0}\right)_{z}(z, t)\right| \leq \varepsilon|z|+C(\varepsilon)|z|^{2}$. Hence

$$
\left|\left\langle\nabla \varphi_{0}(z), y\right\rangle\right| \leq\left(\varepsilon\|z\|+C^{\prime}(\varepsilon)\|z\|^{2}\right)\|y\|
$$

and $\nabla \varphi_{0}(z)=o(\|z\|)$ as $z \rightarrow 0$.

Lemma 7.3. Suppose that $H$ satisfies (7.5). Then $\Phi$ satisfies $(P S)^{*}$ (with respect to any filtration) in each of the following two cases:

(i) $j^{0}(A)=0$;

(ii) $G_{z}$ is bounded and $G(z, t) \rightarrow \infty$ (or $G(z, t) \rightarrow-\infty$ ) uniformly in $t$ as $|z| \rightarrow \infty$.

Moreover, under these hypotheses $\left.\Phi\right|_{E_{n}}$ satisfies (PS) for each $n$.

Proof. (i) Let $\left(z_{j}\right)$ be a (PS)*-sequence. Then $P_{n_{j}} \nabla \Phi\left(z_{j}\right)=P_{n_{j}} L z_{j}-P_{n_{j}} \nabla \varphi\left(z_{j}\right) \rightarrow$ 0 . Since $\nabla \varphi(z)=o(\|z\|)$ as $\|z\| \rightarrow \infty$ and $\left\|P_{n_{j}} L z_{j}\right\| \geq c\left\|z_{j}\right\|$ according to Theorem 4.5, $\left(z_{j}\right)$ is bounded and it follows from the compactness of $\nabla \varphi$ that $\left(z_{j}\right)$ has a convergent subsequence.

(ii) Assume $G(z, t) \rightarrow \infty$ (the other case is similar). Let $\left(z_{j}\right)$ be a (PS)*-sequence and let $z_{j}=y_{j}+w_{j} \in R(L) \oplus N(L)$. Since $P_{n_{j}} L y_{j}-P_{n_{j}} \nabla \varphi\left(z_{j}\right) \rightarrow 0$ and $\left\|P_{n_{j}} L y_{j}\right\| \geq$ $c\left\|y_{j}\right\|$, the sequence $\left(y_{j}\right)$ is bounded. Hence $\varphi\left(z_{j}\right)$ is bounded (because $\Phi\left(z_{j}\right)$ is). By the mean value theorem,

$$
\left|\varphi\left(y_{j}+w_{j}\right)-\varphi\left(w_{j}\right)\right| \leq \sup _{z \in E}\|\nabla \varphi(z)\|\left\|y_{j}\right\| .
$$

So $\varphi\left(w_{j}\right)$ is bounded as well. On the other hand, $\varphi\left(w_{j}\right)=\int_{0}^{2 \pi} G\left(w_{j}(t), t\right) d t \rightarrow \infty$ if $\left\|w_{j}\right\| \rightarrow \infty$ (recall that $N(L)$ is finite dimensional). Hence $\left(w_{j}\right)$ has a convergent subsequence, and the same is true for $\left(y_{j}\right)$ because $\nabla \varphi$ is compact.

Since $E_{n}$ is finite dimensional, it is clear that $\left.\Phi\right|_{E_{n}}$ satisfies (PS).

Theorem 7.4. Suppose that $H$ satisfies (7.5) and (7.6). If $j^{0}(A)=j^{0}\left(A_{0}\right)=0$ and $j^{-}(A) \neq j^{-}\left(A_{0}\right)$, then (7.1) has a nontrivial $2 \pi$-periodic solution.

Proof. It follows immediately from (7.6) that (7.1) has the trivial solution $z=0$. Let $\Phi_{\lambda}(z):=\frac{1}{2}\langle L z, z\rangle-(1-\lambda) \varphi(z), 0 \leq \lambda \leq 1$. It follows from Lemma 7.3 that all $\Phi_{\lambda}$ satisfy (PS)*. Since $L$ is invertible and $\nabla \varphi(z)=o(\|z\|)$ as $\|z\| \rightarrow \infty$, there is a bounded set $N$ such that $K\left(\Phi_{\lambda}\right) \subset N$ and $\sup _{N}\left|\Phi_{\lambda}\right| \leq C$ for some $C>0$ and all $\lambda \in[0,1]$. So according to Propositions 2.12 and 2.14 , the critical groups $c_{\mathcal{E}}^{*}(\Phi, K(\Phi))$ are well-defined and $c_{\mathcal{E}}^{*}(\Phi, K(\Phi))=c_{\mathcal{E}}^{*}\left(\Phi_{1}, K\left(\Phi_{1}\right)\right)=c_{\mathcal{E}}^{*}\left(\Phi_{1}, 0\right)$. 
By Theorem 5.3, $c_{\mathcal{E}}^{q}\left(\Phi_{1}, 0\right)=[\mathcal{F}]$ if $q=j^{-}(A)$ and $c_{\mathcal{E}}^{q}\left(\Phi_{1}, 0\right)=[0]$ otherwise. Since $\nabla \varphi_{0}(z)=o(\|z\|)$ as $z \rightarrow 0$, we obtain -invoking Theorem 5.3 again - that $c_{\mathcal{E}}^{q}(\Phi, 0) \neq[0]$ if and only if $q=j^{-}\left(A_{0}\right)$. So $c_{\mathcal{E}}^{*}(\Phi, K) \neq c_{\mathcal{E}}^{*}(\Phi, 0)$, and $\Phi$ must have a critical point $z \neq 0$.

Theorem 7.5. Suppose that $H \in C^{2}\left(\mathbf{R}^{2 N} \times \mathbf{R}, \mathbf{R}\right)$ satisfies (7.3), (7.5) and (7.6). If $G_{z}$ is bounded, then (7.1) has a nontrivial $2 \pi$-periodic solution in each of the following two cases:

(i) $G(z, t) \rightarrow-\infty$ uniformly in $t$ as $|z| \rightarrow \infty$ and

$$
j^{-}(A) \notin\left[j^{-}\left(A_{0}\right), j^{-}\left(A_{0}\right)+j^{0}\left(A_{0}\right)\right] ;
$$

(ii) $G(z, t) \rightarrow \infty$ uniformly in $t$ as $|z| \rightarrow \infty$ and

$$
j^{+}(A) \notin\left[j^{+}\left(A_{0}\right), j^{+}\left(A_{0}\right)+j^{0}\left(A_{0}\right)\right] .
$$

Proof. (i) Introduce a new filtration $\mathcal{E}^{\prime}:=\left\{E_{n}^{\prime}, d_{n}\right\}_{n=1}^{\infty}$, where $E_{n}^{\prime}:=\left(R(L) \cap E_{n}\right) \oplus$ $N(L)$ and $d_{n}=N(1+2 n)$ as before. According to Lemma 7.3, $\Phi$ satisfies (PS)* with respect to $\mathcal{E}^{\prime}$. Since $R(L) \cap E_{n}^{\prime}=R(L) \cap E_{n}, M_{\mathcal{E}^{\prime}}^{-}(L)=M_{\mathcal{E}}^{-}(L) \equiv j^{-}(A)$. It is easy to see that $L$ and $L_{0}$ (cf. (7.7)) are A-proper with respect to $\mathcal{E}^{\prime}$ (because they are with respect to $\mathcal{E})$. Furthermore, $E_{n}=\left(R(L) \cap E_{n}\right) \oplus P_{n} N(L)$ by Lemma $4.2, E_{n}^{\prime}=\left(R(L) \cap E_{n}\right) \oplus N(L)$ and

$$
\left\|P_{n} z-z\right\| \leq \varepsilon_{n}\|z\| \quad \text { for all } z \in N(L)
$$

where $\varepsilon_{n} \rightarrow 0$ as $n \rightarrow \infty$. Let $Q_{0, n}^{\prime}$ be the orthogonal projector of $R\left(L_{0}\right)$ onto $R\left(L_{0}\right) \cap E_{n}^{\prime}$. It follows from Theorem 4.5 and Proposition 4.3 that there is a $c>0$ such that $\left\|Q_{0, n}^{\prime} L_{0} z\right\| \geq c\|z\|$ for almost all $n$ and all $z \in R\left(L_{0}\right) \cap E_{n}^{\prime}$. Using this and (7.9) we see that the quadratic form $z \mapsto\left\langle L_{0} z, z\right\rangle$ is nondegenerate and has the same Morse index on $R\left(L_{0}\right) \cap E_{n}$ and on $R\left(L_{0}\right) \cap E_{n}^{\prime}$, provided $n$ is sufficiently large. So $M_{\mathcal{E}^{\prime}}^{-}\left(L_{0}\right)=M_{\mathcal{E}}^{-}\left(L_{0}\right) \equiv j^{-}\left(A_{0}\right)$.

In Lemma 7.6 below we will show that if $\Phi$ has finitely many critical points, then (7.5), (i) and the boundedness of $G_{z}$ imply that

$$
c_{\mathcal{E}^{\prime}}^{q}(\Phi, K)= \begin{cases}{[\mathcal{F}]} & \text { if } q=j^{-}(A), \\ {[0]} & \text { otherwise. }\end{cases}
$$

On the other hand, if 0 is an isolated critical point of $\Phi$, it follows from Theorem 5.4 that

$$
c_{\mathcal{E}^{\prime}}^{q}(\Phi, 0)=\left[c^{q-j^{-}\left(A_{0}\right)}\left(\widetilde{\varphi}_{0}, 0\right)\right],
$$

where $\widetilde{\varphi}_{0}$ is defined on a subset of $N\left(L_{0}\right)$. So the right-hand side above can be nonzero only if $0 \leq q-j^{-}\left(A_{0}\right) \leq j^{0}\left(A_{0}\right)$. Since $j^{-}(A) \notin\left[j^{-}\left(A_{0}\right), j^{-}\left(A_{0}\right)+j^{0}\left(A_{0}\right)\right]$,

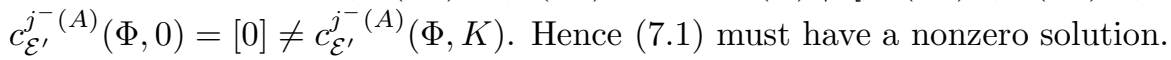

(ii) This follows by the same argument applied to $-\Phi$. (An alternative proof may be obtained by working with $\Phi$, using (ii) of Lemma 7.6 and the fact that $j^{-}(A)+j^{+}(A)+j^{0}(A)=0$.)

Observe that if the matrix $A$ is $t$-independent, then $N(L) \subset E_{n_{0}}$ for some $n_{0}$ and $E_{n}=E_{n}^{\prime}$ for almost all $n$. So in this case we can use the filtration $\mathcal{E}$.

Lemma 7.6. Suppose that $\Phi \in C^{1}\left(\mathbf{R}^{2 N} \times \mathbf{R}, \mathbf{R}\right)$ satisfies (7.5), $G_{z}$ is bounded and the critical set $K=K(\Phi)$ is finite.

(i) If $G(z, t) \rightarrow-\infty$ uniformly in $t$ as $|z| \rightarrow \infty$, then $c_{\mathcal{E}^{\prime}}^{*}(\Phi, K)$ is given by (7.10). 
(ii) If $G(z, t) \rightarrow \infty$ uniformly in $t$ as $|z| \rightarrow \infty$, then

$$
c_{\mathcal{E}^{\prime}}^{q}(\Phi, K)= \begin{cases}{[\mathcal{F}]} & \text { if } q=j^{-}(A)+j^{0}(A), \\ {[0]} & \text { otherwise. }\end{cases}
$$

Proof. (i) Let $Q_{n}$ be the orthogonal projector of $R(L)$ onto $R(L) \cap E_{n}$. Find $c>0$ and $n_{0}$ such that $\left\|Q_{n} L z\right\| \geq c\|z\|$ for all $z \in R(L) \cap E_{n}$ and $n \geq n_{0}$. Let $E_{n}^{\prime} \equiv(R(L) \cap E) \oplus N(L)=E_{n}^{+} \oplus E_{n}^{-} \oplus N(L)$ be the decomposition (into $L$ invariant subspaces) corresponding to the positive, the negative and the zero part of the quadratic form $z \mapsto\langle L z, z\rangle$ on $E_{n}^{\prime}$. If $z=z^{+}+z^{-}+z^{0} \in E_{n}^{+} \oplus E_{n}^{-} \oplus N(L)$, then $\left\langle L z^{+}, z^{+}\right\rangle \geq c\left\|z^{+}\right\|^{2}$ and $\left\langle L z^{-}, z^{-}\right\rangle \leq-c\left\|z^{-}\right\|^{2}$. Therefore

$$
\left\langle\nabla \Phi(z), z^{-}\right\rangle=\left\langle L z^{-}, z^{-}\right\rangle-\left\langle\nabla \varphi(z), z^{-}\right\rangle \leq-c\left\|z^{-}\right\|^{2}+C_{0}\left\|z^{-}\right\|,
$$

where $C_{0}:=\sup _{E}\|\nabla \varphi(z)\|$. Hence there is an $R>0$ such that setting

$$
U:=\left\{z \in E_{n}^{\prime}:\left\|z^{-}\right\|<R\right\}
$$

we obtain

$$
\left\langle\nabla \Phi(z), z^{-}\right\rangle<0 \quad \text { for all } z \in E_{n}^{\prime}-U \text { and } n \geq n_{0} .
$$

In particular, $\left.\Phi\right|_{E_{n}^{\prime}}$ has no critical points in $E_{n}^{\prime}-U$. For $z \in \bar{U}$ we have

$$
\begin{aligned}
\Phi(z) & =\frac{1}{2}\left\langle L z^{+}, z^{+}\right\rangle+\frac{1}{2}\left\langle L z^{-}, z^{-}\right\rangle-\varphi(z) \\
& \geq \frac{1}{2} c\left\|z^{+}\right\|^{2}-\frac{1}{2}\|L\| R^{2}-\varphi\left(z^{0}\right)-\left(\varphi(z)-\varphi\left(z^{0}\right)\right) \\
& \geq \frac{1}{2} c\left\|z^{+}\right\|^{2}-\frac{1}{2}\|L\| R^{2}-\varphi\left(z^{0}\right)-C_{0}\left(R+\left\|z^{+}\right\|\right) .
\end{aligned}
$$

Since $\varphi\left(z^{0}\right) \rightarrow-\infty$ as $\left\|z^{0}\right\| \rightarrow \infty$, it follows that

$$
\Phi(z) \rightarrow \infty \quad \text { as }\left\|z^{+}+z^{0}\right\| \rightarrow \infty
$$

and the convergence is uniform with respect to the choice of $n \geq n_{0}$ and $z^{-} \in$ $E_{n}^{-} \cap \bar{B}(0, R)$. Hence we can find $0<a<b$ and $R_{0}>0$ such that $K \subset\{z \in E$ : $|\Phi(z)|<a\}$, and for each $n \geq n_{0}$,

$\Phi^{-a} \cap E_{n}^{\prime} \subset E_{n}^{\prime}-U \quad$ and $\quad \Phi^{a} \cap \bar{U} \subset D:=\left\{z \in \bar{U}:\left\|z^{+}+z^{0}\right\| \leq R_{0}\right\} \subset \Phi^{b} \cap \bar{U}$.

It is easy to see that there exists a strong deformation retraction $\gamma$ of $E_{n}^{\prime}$ onto $D \cup \partial U$. Using (7.11) we may construct a pseudogradient vector field $V$ for $\Phi$ on $E_{n}^{\prime}$ in such a way that $\left\langle V(z), z^{-}\right\rangle<0$ whenever $\left\|z^{-}\right\| \geq R$. It follows from $(\mathrm{PS})^{*}$ that if $n$ is large enough, then $\left.\Phi\right|_{E_{n}^{\prime}}$ has no critical values in $[a, b]$. So $K\left(\left.\Phi\right|_{E_{n}^{\prime}}\right) \subset U-\Phi^{-1}([a, b])$. Since $\left.\Phi\right|_{E_{n}^{\prime}}$ satisfies (PS), the flow of $-V$ induces a strong deformation retraction $\eta$ of $\left(E_{n}^{\prime}-U\right) \cup D$ onto $\Phi^{a} \cap E_{n}^{\prime}$. Indeed, if $z \in E_{n}^{\prime}-U$, then $\eta(\lambda, z) \in E_{n}^{\prime}-U$ (because $\left.\left\langle V(z), z^{-}\right\rangle<0\right)$ and if $z \in D$, then $\eta(\lambda, z) \in \Phi^{b} \cap U$ (because $\left.D \subset \Phi^{b} \cap \bar{U}\right)$. So $\eta$ may be constructed in such a way that it is a deformation onto $\Phi^{a} \cap E_{n}^{\prime}$. Now we see that the mapping $\eta * \gamma$ given by

$$
(\eta * \gamma)(\lambda, z):= \begin{cases}\gamma(2 \lambda, z) & \text { for } 0 \leq \lambda \leq \frac{1}{2} \\ \eta(2 \lambda-1, \gamma(1, z)) & \text { for } \frac{1}{2} \leq \lambda \leq 1\end{cases}
$$

is a strong deformation retraction of $E_{n}^{\prime}$ onto $\Phi^{a} \cap E_{n}^{\prime}$. Since $\Phi^{-a} \cap E_{n}^{\prime} \subset E_{n}^{\prime}-U$ and $\left\langle V(z), z^{-}\right\rangle<0$ as $\left\|z^{-}\right\| \geq R$, one can use the flow of $-V$ again in order to 
construct a strong deformation retraction of $E_{n}^{\prime}-U$ onto $\Phi^{-a} \cap E_{n}^{\prime}$. Therefore

$$
H^{q}\left(\Phi^{a} \cap E_{n}^{\prime}, \Phi^{-a} \cap E_{n}^{\prime}\right) \cong H^{q}\left(E_{n}^{\prime}, E_{n}^{\prime}-U\right)= \begin{cases}\mathcal{F} & \text { if } q=j^{-}(A)+d_{n} \\ 0 & \text { otherwise }\end{cases}
$$

provided $n$ is large enough. Since $H_{\mathcal{E}^{\prime}}^{*}\left(\Phi^{a}, \Phi^{-a}\right) \cong H_{\mathcal{E}^{\prime}}^{*}\left(\Phi^{-1}([-a, a]), \Phi^{-1}(-a)\right)$ (by excision) and the second pair is admissible for $\Phi$ and $K$, the conclusion follows.

(ii) Here we have

$$
\left\langle\nabla \Phi(z), z^{+}\right\rangle \geq c\left\|z^{+}\right\|^{2}-C_{0}\left\|z^{+}\right\|,
$$

and we may choose $R$ such that if

$$
M:=\left\{z \in E_{n}^{\prime}:\left\|z^{+}\right\| \leq R\right\}
$$

then

$$
\left\langle\nabla \Phi(z), z^{+}\right\rangle>0 \text { for all } z \in E_{n}^{\prime}-\operatorname{int}(M) \text { and } n \geq n_{0} .
$$

As in (7.12), we obtain

$$
\Phi(z) \leq \frac{1}{2}\|L\| R^{2}-\frac{1}{2} c\left\|z^{-}\right\|^{2}-\varphi\left(z_{0}\right)+C_{0}\left(R+\left\|z^{-}\right\|\right)
$$

for all $z \in M$. Therefore

$$
\Phi(z) \rightarrow-\infty \quad \text { as }\left\|z^{-}+z^{0}\right\| \rightarrow \infty,
$$

and the convergence is uniform with respect to the choice of $n \geq n_{0}$ and $z^{+} \in$ $E_{n}^{+} \cap \bar{B}(0, R)$. Hence we can find $0<a<b$ and $0<R_{2}<R_{1}$ such that $K \subset\{z \in$ $E:|\Phi(z)|<a\}, M \subset \Phi^{a} \cap E_{n}^{\prime}$ and

$$
\begin{aligned}
D_{1} & :=\left\{z \in M:\left\|z^{-}+z^{0}\right\| \geq R_{1}\right\} \subset \Phi^{-b} \cap M \\
& \subset D_{2}:=\left\{z \in M:\left\|z^{-}+z^{0}\right\| \geq R_{2}\right\} \subset \Phi^{-a} \cap M .
\end{aligned}
$$

Obviously, there exists a strong deformation retraction $\gamma$ of $D_{2}$ onto $D_{1}$. By (PS)*, we may assume that $K\left(\left.\Phi\right|_{E_{n}^{\prime}}\right) \subset M-\Phi^{-1}([-b,-a])$. Using the flow of $-V$, where $V$ is a pseudogradient vector field on $E_{n}^{\prime}$ satisfying $\left\langle V(z), z^{+}\right\rangle>0$ for $\left\|z^{+}\right\| \geq R$, it is easy to construct a strong deformation retraction $\eta$ of $\Phi^{-a} \cap M$ onto $\Phi^{-\bar{b}} \cap M$. Hence $\gamma * \eta$ is a strong deformation retraction of $\Phi^{-a} \cap M$ onto $D_{1}$. Using the flow of $-V$ once again, we also obtain a strong deformation retraction of $\Phi^{a} \cap E_{n}^{\prime}$ onto $\left(\Phi^{-a} \cap E_{n}^{\prime}\right) \cup M$. Hence

$$
\begin{aligned}
H^{*}\left(\Phi^{a} \cap E_{n}^{\prime}, \Phi^{-a} \cap E_{n}^{\prime}\right) & \cong H^{*}\left(\left(\Phi^{-a} \cap E_{n}^{\prime}\right) \cup M, \Phi^{-a} \cap E_{n}^{\prime}\right) \\
& \stackrel{e x c}{\cong} H^{*}\left(M, \Phi^{-a} \cap M\right) \cong H^{*}\left(M, D_{1}\right) .
\end{aligned}
$$

Since for all $n$ large enough,

$$
H^{q}\left(M, D_{1}\right)= \begin{cases}\mathcal{F} & \text { if } q=j^{-}(A)+j^{0}(A)+d_{n}, \\ 0 & \text { otherwise }\end{cases}
$$

we obtain the conclusion.

It is clear that if $j^{0}\left(A_{0}\right)=0$, then it suffices to assume that $H \in C^{1}$ in Theorem 7.5, and if $j^{0}(A)=0, G_{z}$ need not be bounded and $G$ need not tend to infinity.

Suppose now that $H \in C^{2}\left(\mathbf{R}^{2 N} \times \mathbf{R}, \mathbf{R}\right)$ satisfies $(7.5)$ and $z_{0}$ is a $2 \pi$-periodic solution of (7.1). Then $z_{0}$ is continuous (and therefore bounded). Hence for each $\varepsilon>0$ there is a constant $C(\varepsilon)$ such that

$$
\left|G_{z}\left(z_{0}(t)+w, t\right)-G_{z}\left(z_{0}(t), t\right)-G_{z z}\left(z_{0}(t), t\right) w\right| \leq \varepsilon|w|+C(\varepsilon)|w|^{2}
$$


for all $t \in \mathbf{R}$ and $w \in \mathbf{R}^{2 N}$. So

$$
\left|\left\langle\nabla \varphi\left(z_{0}+v\right)-\nabla \varphi\left(z_{0}\right), y\right\rangle-\int_{0}^{2 \pi} G_{z z}\left(z_{0}, t\right) v \cdot y d t\right| \leq\left(\varepsilon\|v\|+C^{\prime}(\varepsilon)\|v\|^{2}\right)\|y\|
$$

for all $v, y \in E$. Dividing by $\|v\|$ and letting $v \rightarrow 0$, we see that $\nabla \varphi$ is Fréchet differentiable at $z_{0}$ and

$$
\Phi\left(z_{0}+v\right)=\Phi\left(z_{0}\right)+\frac{1}{2}\left\langle\Phi^{\prime \prime}\left(z_{0}\right) v, v\right\rangle+\psi\left(z_{0}+v\right)
$$

where $\nabla \psi\left(z_{0}+v\right)=o(\|v\|)$ as $v \rightarrow 0$. Note that we made no assumption that $H$ satisfies (7.3), and therefore $\Phi$ may not be of class $C^{2}$. We will call the solution $z_{0}$ nondegenerate if $\Phi^{\prime \prime}\left(z_{0}\right)$ is invertible.

Remark 7.7. Suppose that $H \in C^{2}\left(\mathbf{R}^{2 N} \times \mathbf{R}, \mathbf{R}\right)$ satisfies the hypotheses of Theorem 7.4. If the nontrivial solution $z_{0}$ of $(7.1)$ is nondegenerate, then (7.1) has a second nontrivial solution. Indeed, suppose 0 and $z_{0}$ are the only solutions. According to Theorem 5.3, their contribution to the Morse polynomial is respectively $t^{j^{-}\left(A_{0}\right)}$ and $t^{q_{0}}$, where $q_{0} \in \mathbf{Z}$. So the Morse inequalities give

$$
t^{j^{-}\left(A_{0}\right)}+t^{q_{0}}=t^{j^{-}(A)}+(1+t) Q(t)
$$

a contradiction upon setting $t=1$. In the framework of Theorem 7.5 the above conclusion remains valid if $j^{0}\left(A_{0}\right)=0$ (note that (7.3) is not needed here).

The nondegeneracy condition for $z_{0}$ in Remark 7.7 is in general not easy to verify. However, if the difference between the indices $j^{-}(A)$ and $j^{-}\left(A_{0}\right)$ is sufficiently large, this condition can be avoided.

Theorem 7.8. Suppose that $H \in C^{2}\left(\mathbf{R}^{2 N} \times \mathbf{R}, \mathbf{R}\right)$ satisfies (7.3), (7.5), (7.6) and $j^{0}\left(A_{0}\right)=0$. Then (7.1) has at least two nontrivial $2 \pi$-periodic solutions in each of the following cases:

(i) $\left|j^{-}(A)-j^{-}\left(A_{0}\right)\right| \geq 2 N$ and $j^{0}(A)=0$;

(ii) $\left|j^{-}(A)-j^{-}\left(A_{0}\right)\right| \geq 2 N, G_{z}$ is bounded and $G(z, t) \rightarrow-\infty$ uniformly in $t$ as $|z| \rightarrow \infty$

(iii) $\left|j^{+}(A)-j^{+}\left(A_{0}\right)\right| \geq 2 N, G_{z}$ is bounded and $G(z, t) \rightarrow \infty$ uniformly in $t$ as $|z| \rightarrow \infty$.

Proof. Assume that (i) or (ii) is satisfied. Let $z_{0}$ be the nontrivial solution we already know exists, and suppose there are no other ones. According to Theorem 5.4 ,

$$
c_{\mathcal{E}^{\prime}}^{q}\left(\Phi, z_{0}\right)=\left[c^{q-r_{0}}\left(\widetilde{\psi}_{0}, 0\right)\right]
$$

for some $r_{0} \in \mathbf{Z}$ and some functional $\widetilde{\psi}_{0}$ defined on a space $Z$ of dimension $\leq 2 N$. So $c_{\mathcal{E}^{\prime}}^{q}\left(\Phi, z_{0}\right)$ can be nonzero only for $0 \leq q-r_{0} \leq \operatorname{dim} Z \leq 2 N$. Moreover, if $\widetilde{\psi}_{0}$ has a local minimum at 0 , then $c^{q-r_{0}}\left(\widetilde{\psi}_{0}, 0\right) \neq 0$ if and only if $q-r_{0}=0$; if it has a local maximum, then $c^{q-r_{0}}\left(\widetilde{\psi}_{0}, 0\right) \neq 0$ if and only if $q-r_{0}=\operatorname{dim} Z$; and in other cases $c^{0}\left(\widetilde{\psi}_{0}, 0\right)=c^{\operatorname{dim} Z}\left(\widetilde{\psi}_{0}, 0\right)=0$ (see [15] or [34, Theorem 8.6 and Corollary 8.4]). Consequently, the Morse inequalities give

$$
t^{j^{-}\left(A_{0}\right)}+\sum_{i=0}^{2 N-2} b_{i} t^{\alpha+i}=t^{j^{-}(A)}+(1+t) Q(t)
$$

where $b_{i} \in[\mathbf{Z}]$ and some (or all) $b_{i}$ may be zero and $\alpha \in \mathbf{Z}$. Since there is an exponent $j^{-}(A)$ on the right-hand side above, $\alpha+i=j^{-}(A)$ for some $i$. The 
left-hand side contains the exponent $j^{-}\left(A_{0}\right)$. Therefore $Q(t)$ must have a nonzero term with exponent $j^{-}\left(A_{0}\right)$ or $j^{-}\left(A_{0}\right)-1$, and it follows that there is a nonzero term with exponent $j^{-}\left(A_{0}\right)-1$ or $j^{-}\left(A_{0}\right)+1$ on the left-hand side. Hence there exists $j, 0 \leq j \leq 2 N-2$, such that $\alpha+j=j^{-}\left(A_{0}\right)+1$ or $j^{-}\left(A_{0}\right)-1$. So $\left|j^{-}(A)-j^{-}\left(A_{0}\right)\right|=|i-j \pm 1| \leq 2 N-1$, a contradiction.

Finally, if (iii) is satisfied, the conclusion is obtained by applying the same argument to $-\Phi$.

Corollary 7.9. Suppose that $H \in C^{2}\left(\mathbf{R}^{2 N} \times \mathbf{R}, \mathbf{R}\right)$ satisfies (7.3), $H_{z}$ is bounded and $H_{z}(z, t)=o(z)$ uniformly in $t$ as $z \rightarrow 0$. Then (7.1) has at least two nontrivial $2 \pi$-periodic solutions in each of the following two cases:

(i) $H(z, t) \rightarrow-\infty$ uniformly in $t$ as $|z| \rightarrow \infty$ and there is a $\delta>0$ such that $H(z, t) \geq 0$ whenever $|z| \leq \delta$

(ii) $H(z, t) \rightarrow \infty$ uniformly in $t$ as $|z| \rightarrow \infty$ and there is a $\delta>0$ such that $H(z, t) \leq 0$ whenever $|z| \leq \delta$.

Proof. (i) Note that $H$ satisfies (7.5) and (7.6) with $A=A_{0} \equiv 0$. Suppose that $z_{0}$ is the only nontrivial solution of (7.1). Since $j^{-}(0)=i^{-}(0)=-N$, it follows from (7.10) (with $\mathcal{E}^{\prime}=\mathcal{E}$ ) that $c_{\mathcal{E}}^{q}(\Phi, K)=[\mathcal{F}]$ if $q=-N$ and [0] otherwise. Furthermore, $j^{0}(0)=2 N$, so according to Corollary $5.5, c_{\mathcal{E}}^{q}(\Phi, 0)=[\mathcal{F}]$ if $q=N$ and $[0]$ otherwise (observe that $\varphi$ in Corollary 5.5 corresponds to $-\psi$ here, cf. (7.2)). Hence we obtain from the Morse inequalities that

$$
t^{N}+\sum_{i=0}^{2 N-2} b_{i} t^{\alpha+i}=t^{-N}+(1+t) Q(t)
$$

which leads to a contradiction in the same way as in the proof of Theorem 7.8.

(ii) The argument is similar except that now $c_{\mathcal{E}}^{N}(\Phi, K)=[\mathcal{F}]$ and $c_{\mathcal{E}}^{-N}(\Phi, 0)=[\mathcal{F}]$ according to (ii) of Lemma 7.6 and Corollary 5.5.

If $G_{0}$ has constant sign for $z$ in a neighbourhood of the origin in $\mathbf{R}^{2 N}$, a better result than Theorem 7.5 can be obtained.

Theorem 7.10. Suppose that $H$ satisfies (7.5) and (7.6) with $A$ and $A_{0}$ independent of $t$ and either $j^{0}(A)=0$ or $G_{z}$ is bounded and $G(z, t) \rightarrow-\infty$ uniformly in $t$ as $|z| \rightarrow \infty$. Then (7.1) has a nontrivial $2 \pi$-periodic solution in each of the following two cases:

(i) $j^{-}(A) \neq j^{-}\left(A_{0}\right)+j^{0}\left(A_{0}\right)$ and there is a $\delta>0$ such that $G_{0}(z, t) \geq 0$ whenever $|z| \leq \delta$

(ii) $j^{-}(A) \neq j^{-}\left(A_{0}\right)$ and there is a $\delta>0$ such that $G_{0}(z, t) \leq 0$ whenever $|z| \leq \delta$.

If $A, A_{0}$ are $t$-dependent, the same conclusion remains valid provided $H \in$ $C^{2}\left(\mathbf{R}^{2 N} \times \mathbf{R}, \mathbf{R}\right)$ and satisfies (7.3).

Proof. (i) Suppose that 0 is the only critical point of $\Phi$ and let $E=Y \oplus Z$ be the decomposition corresponding to the nonpositive and the positive part of the spectrum of $L_{0}$. It has been shown in [26], cf. also [28, 29], that $\Phi$ satisfies the local linking condition at 0 with $Y$ and $Z$ as above. Moreover, since $A_{0}$ is independent of $t, L_{0}\left(F_{k}\right) \subset F_{k}$ for each $k$ and therefore $E_{n}=\left(Y \cap E_{n}\right) \oplus\left(Z \cap E_{n}\right)$. Also, since $N\left(L_{0}\right) \subset E_{n}$, it is easy to see that $\operatorname{dim}\left(Y \cap E_{n}\right)=j^{-}\left(A_{0}\right)+j^{0}\left(A_{0}\right)+d_{n}$ for almost all $n$. Hence in view of Theorem 5.6, $c_{\mathcal{E}}^{j^{-}\left(A_{0}\right)+j^{0}\left(A_{0}\right)}(\Phi, 0) \neq[0]$. On the other hand, $c_{\mathcal{E}}^{q}(\Phi, K) \neq[0]$ if and only if $q=j^{-}(A)$ (this follows from the proof of Theorem 7.4 
if $j^{0}(A)=0$ and from (7.10) otherwise). Since $j^{-}(A) \neq j^{-}\left(A_{0}\right)+j^{0}\left(A_{0}\right), \Phi$ must have a nontrivial critical point.

If $A, A_{0}$ are $t$-dependent and $H$ satisfies (7.3), we use the filtration $\mathcal{E}^{\prime}$ and obtain from Corollary 5.5 that $c_{\mathcal{E}^{\prime}}^{j^{-}\left(A_{0}\right)+j^{0}\left(A_{0}\right)}(\Phi, 0)=[\mathcal{F}](\varphi$ in corollary 5.5 corresponds to $-\varphi_{0}$ here). This contradicts the fact that $c_{\mathcal{E}^{\prime}}^{q}(\Phi, 0)=c_{\mathcal{E}^{\prime}}^{q}(\Phi, K) \neq[0]$ if and only if $q=j^{-}(A)$.

(ii) The proof is the same except that now $Y$ corresponds to the negative and $Z$ to the nonnegative part of the spectrum of $L_{0}$. So $\operatorname{dim}\left(Y \cap E_{n}\right)=j^{-}\left(A_{0}\right)+d_{n}$ for almost all $n$.

If $G(z, t) \rightarrow \infty$ as $|z| \rightarrow \infty$, a similar result can be formulated in terms of $j^{+}(A)$ and $j^{+}\left(A_{0}\right)$.

Suppose now that

$$
\begin{aligned}
& H(z, t)=\frac{1}{2} A(t) p \cdot p+G(z, t), \\
& \quad \text { where } G_{z}(z, t)=o(|z|) \text { uniformly in } t \text { as }|z| \rightarrow \infty,
\end{aligned}
$$

$z=(p, q) \in \mathbf{R}^{N} \times \mathbf{R}^{N}, A(t)$ is a symmetric $N \times N$ matrix with $2 \pi$-periodic entries and $G$ is $2 \pi$-periodic in $q$ and $t$. If $z$ is a $2 \pi$-periodic solution of (7.1), so are all $\widetilde{z}=(p, \widetilde{q})$ with $\widetilde{q} \equiv q(\bmod 2 \pi)$. Hence to each solution $z$ there corresponds an orbit $\mathcal{O}(z):=\left\{z+\left(0,2 \pi \mathbf{Z}^{N}\right)\right\}$. Two solutions $z_{1}, z_{2}$ are called geometrically distinct if $\mathcal{O}\left(z_{1}\right) \cap \mathcal{O}\left(z_{2}\right)=\emptyset$. Let $E=\widetilde{E} \oplus N$, where $N:=\left\{(p, q): p=0, q \in \mathbf{R}^{N}\right\}$ and $\widetilde{E}=N^{\perp}$. So $N$ is the subspace of constant functions $(p, q)$ such that $p=0$ and $\widetilde{E}$ is the subspace of functions in $E$ whose $q$-coordinates have mean value zero. Let $z=x+v \in \widetilde{E} \oplus N$ and define (cf. (7.7))

$$
\Phi(x, v):=\frac{1}{2} \int_{0}^{2 \pi}(-J \dot{x} \cdot x-A(t) p \cdot p) d t-\int_{0}^{2 \pi} G(z, t) d t=\frac{1}{2}\langle L x, x\rangle-\varphi(x, v) .
$$

Then $\Phi: \widetilde{E} \times N \rightarrow \mathbf{R}$. Since $\Phi(x, v)=\Phi(x, \widetilde{v})$ if $\widetilde{v} \equiv v(\bmod 2 \pi), v$ may be regarded as an element of the torus $T^{N}:=\mathbf{R}^{N} / 2 \pi \mathbf{Z}^{N}$ and $\Phi$ maps $M:=E \times T^{N}$ into R. Moreover, distinct critical points of $\Phi$ on $M$ correspond to geometrically distinct $2 \pi$-periodic solutions of (7.1).

One sees that $L x=0$ if and only if $\dot{p}=0$ and $\dot{q}=A(t) p$. So $N(L)$ consists of $(p, q) \in \widetilde{E}$ such that $p \in \mathbf{R}^{N}, \dot{q}=A(t) p$ and $A(t) p$ has mean value zero. In what follows we assume for simplicity that $L$ is invertible on $\widetilde{E}$. As in [41], this assumption may be relaxed by requiring that if $N(L)$ is nontrivial, then $G_{z}$ is bounded and $G(p, q, t) \rightarrow \infty$ (or $-\infty)$ uniformly in $(q, t)$ as $|p| \rightarrow \infty, p \in P_{1} N(L)$ ( $P_{1}$ is the projector onto the first component of $x=(p, q)$ ).

Theorem 7.11. Suppose that $H \in C^{2}\left(\mathbf{R}^{2 N} \times \mathbf{R}, \mathbf{R}\right)$ is $2 \pi$-periodic in $q, t$ and satisfies (7.13). If $L$ is invertible on $\widetilde{E}$ and all $2 \pi$-periodic solutions of (7.1) are nondegenerate, then the number of geometrically distinct ones is at least $2^{N}$.

Proof. Let $\mathcal{E}:=\left\{M_{n}, d_{n}\right\}$, where $M_{n}:=\left(\widetilde{E} \cap E_{n}\right) \times T^{N}$ (cf. Remark 2.15). Suppose that $\Phi$ has finitely many critical points (otherwise there is nothing to prove). Using Propositions 2.12 and 2.14 in the same way as in the proof of Theorem 7.4, we see that $c_{\mathcal{E}}^{*}(\Phi, K(\Phi))=c_{\mathcal{E}}^{*}\left(\Phi_{1}, K\left(\Phi_{1}\right)\right)$, where $\Phi_{1}(x, v):=\frac{1}{2}\langle L x, x\rangle$. Moreover, if $\left(\widetilde{W}, \widetilde{W}^{-}\right)$is a bounded admissible pair for $x \mapsto \frac{1}{2}\langle L x, x\rangle$ and 0 on $\widetilde{E}$, then $\left(W, W^{-}\right):=\left(\widetilde{W}, \widetilde{W}^{-}\right) \times T^{N}$ is admissible for $\Phi_{1}$ and $K\left(\Phi_{1}\right)$. Since $L$ is invertible on 
$\widetilde{E}$, there exists a unique $q_{0}$ such that for almost all $n, H^{q+d_{n}}\left(\widetilde{W} \cap E_{n}, \widetilde{W}^{-} \cap E_{n}\right)=\mathcal{F}$ if $q=q_{0}$ and $=0$ otherwise. So it follows from the Künneth formula [17, Proposition VI.12.16], [39, Theorem 5.6.1] that

$$
H^{q+d_{n}}\left(W \cap E_{n}, W^{-} \cap E_{n}\right)=\left\{H^{*}\left(\widetilde{W} \cap E_{n}, \widetilde{W}^{-} \cap E_{n}\right) \otimes H^{*}\left(T^{N}\right)\right\}^{q+d_{n}}=H^{q-q_{0}}\left(T^{N}\right) .
$$

Hence

$$
c_{\mathcal{E}}^{*}(\Phi, K(\Phi))=H_{\mathcal{E}}^{*}\left(W, W^{-}\right)=\left[H^{*-q_{0}}\left(T^{N}\right)\right],
$$

and since $H^{*}\left(T^{N}\right)=H^{*}\left(S^{1}\right) \otimes \cdots \otimes H^{*}\left(S^{1}\right)(N$ times $)$,

$$
\beta_{\mathcal{E}}^{q}\left(W, W^{-}\right) \equiv \operatorname{dim}_{\mathcal{E}} H_{\mathcal{E}}^{q}\left(W, W^{-}\right)=\left[\left(\begin{array}{c}
N \\
q-q_{0}
\end{array}\right)\right] \quad \text { for } q_{0} \leq q \leq q_{0}+N
$$

and $\beta_{\mathcal{E}}^{q}\left(W, W^{-}\right)=[0]$ otherwise. We have assumed that all critical points of $\Phi$ are nondegenerate. Locally we may identify $\Phi$ on $M=\widetilde{E} \times T^{N}$ with the same functional on $E$. It follows therefore as in Remark 7.7 that each critical point $\left(x_{i}, v_{i}\right)$ of $\Phi$ on $M$ contributes with a term $t^{q_{i}}$ to the Morse polynomial. Since all coefficients $a_{q}$ in the polynomial $Q$ in the Morse inequalities are nonnegative (in the sense that $\left.a_{q} \in\left[\mathbf{Z}_{+}\right]\right)$, it follows from Theorem 3.1 that

$$
M_{\mathcal{E}}^{q}\left(\hat{W}, \hat{W}^{-}\right) \geq \beta_{\mathcal{E}}^{q}\left(\hat{W}, \hat{W}^{-}\right)
$$

where the relation $\geq$ has the obvious meaning and $\left(\hat{W}, \hat{W}^{-}\right)$is a (globally) admissible pair for $\Phi$ and $K(\Phi)$. So if $m$ is the number of critical points of $\Phi$, then

$$
[m]=\sum_{q \in \mathbf{Z}} M_{\mathcal{E}}^{q}\left(\hat{W}, \hat{W}^{-}\right) \geq \sum_{q \in \mathbf{Z}} \beta_{\mathcal{E}}^{q}\left(\hat{W}, \hat{W}^{-}\right)=\sum_{q=q_{0}}^{q_{0}+N}\left[\left(\begin{array}{c}
N \\
q-q_{0}
\end{array}\right)\right]=\left[2^{N}\right] .
$$

Remark 7.12. (i) Theorems 7.4, 7.5 and 7.10 extend different results contained in $[1,2,13,26,32,41]$. In $[1,2]$ it was assumed that $H_{z z}$ is bounded, $A, A_{0}$ are timeindependent and $j^{0}(A)=0$, in [13] $H_{z z}$ is bounded and $j^{0}(A)=j^{0}\left(A_{0}\right)=0$, in [26] $A, A_{0}$ are time-independent and $j^{0}(A)=0$, in [32] $H_{z z}$ is bounded and $j^{0}(A)=0$, and in [41] $A, A_{0}$ are time-independent. Theorem 7.4, with a different proof, may also be found in [10, p. 186]. Theorem 7.8 extends a result by Bertotti [7], where it was assumed that $H_{z z}$ is bounded and $j^{0}(A)=0$ (see also [32]), and Theorem 7.11 is a generalization of a result in [19] and [41] (in [19] $H_{z z}$ is bounded and $A$ time-independent, and in [41] $A$ is time-independent).

The assumption that $H_{z z}$ is bounded-which has been made in some of the work mentioned above - was needed in order to reduce the problem to a finite dimensional one. After this reduction the usual Morse theory was employed.

(ii) If $A=0, G$ is $2 \pi$-periodic in all variables and all solutions of (7.1) are nondegenerate, then the number of geometrically distinct ones is at least $2^{2 N}$. This follows by an easy modification of the proof of Theorem 7.11. Thus we recover the celebrated result by Conley and Zehnder on Arnold's conjecture [12], see also [10].

(iii) If the nondegeneracy assumption in Theorem 7.11 is removed and if $H \in$ $C^{1}\left(\mathbf{R}^{2 N} \times \mathbf{R}, \mathbf{R}\right)$, then (7.1) has at least $N+1$ geometrically distinct $2 \pi$-periodic solutions $[18,30,40]$. 


\section{WAVE EQUATION}

In this section we are concerned with the existence of nontrivial solutions of the wave equation (0.3) satisfying the boundary and the periodicity conditions (0.4). More precisely, we consider the problem

$$
\begin{cases}\square u:=u_{t t}-u_{x x}=f(x, t, u), & 0<x<\pi, t \in \mathbf{R}, \\ u(0, t)=u(\pi, t)=0, & t \in \mathbf{R}, \\ u(x, t+2 \pi)=u(x, t), & 0<x<\pi, t \in \mathbf{R},\end{cases}
$$

with $f$ satisfying the following hypotheses:

$$
\begin{aligned}
& f \in C\left([0, \pi] \times \mathbf{R}^{2}, \mathbf{R}\right) \text {, and } f(x, t+2 \pi, \xi)=f(x, t, \xi) \\
& \text { for all } x, t, \xi, \\
& \text { There exists an } \varepsilon>0 \text { such that }(f(x, t, \xi)-f(x, t, \eta))(\xi-\eta) \\
& \geq \varepsilon(\xi-\eta)^{2} \text { for all } x, t, \xi, \eta, \\
& f(x, t, \xi)=b \xi+g(x, t, \xi), \text { where } g(x, t, \xi)=o(|\xi|) \\
& \quad \text { uniformly in }(x, t) \text { as }|\xi| \rightarrow \infty, \\
& f(x, t, \xi)=b_{0} \xi+g_{0}(x, t, \xi), \text { where } g_{0}(x, t, \xi)=o(|\xi|) \\
& \text { uniformly in }(x, t) \text { as } \xi \rightarrow 0 .
\end{aligned}
$$

It follows from (8.2)-(8.5) that the constants $b_{0}, b$ are positive and $u=0$ is a solution of (8.1) (the trivial solution).

Let $\Omega:=(0, \pi) \times(0,2 \pi)$ and let $E$ be the space of functions

$$
u(x, t)=\sum_{j=1}^{\infty} \sum_{k=-\infty}^{\infty} c_{j k} \sin j x \mathrm{e}^{i k t}, \quad c_{j,-k}=\bar{c}_{j k},
$$

such that $\sum_{j \neq|k|}\left|j^{2}-k^{2}\right|\left|c_{j k}\right|^{2}+\sum_{j=|k|}\left|c_{j k}\right|^{2}<\infty$. Then $E$ is a Hilbert space with inner product

$$
\left\langle u, u^{\prime}\right\rangle:=\pi^{2} \sum_{j \neq|k|}\left|j^{2}-k^{2}\right| c_{j k} \bar{c}_{j k}^{\prime}+\pi^{2} \sum_{j=|k|} c_{j k} \bar{c}_{j k}^{\prime} .
$$

Observe that the basis $\left\{\sin j x \mathrm{e}^{i k t}\right\}$ for $E$ consists of eigenfunctions of the wave operator $\square$. In what follows \|\| will denote the norm in $E$ and \|\|$_{p}$ the norm in $L^{p}(\Omega), 1 \leq p \leq \infty$.

Let

$$
N:=\left\{u \in E: u(x, t)=\sum_{j=|k|} c_{j k} \sin j x \mathrm{e}^{i k t}\right\}
$$

and denote the orthogonal complement of $N$ in $E$ by $N^{\perp}$. Let $u \in E$. Then $\square u=0$ (in the sense of distributions) if and only if $u \in N$. So $N$ is the (generalized) nullspace of the operator $\square$ subject to our boundary and periodicity conditions. It follows from the Fourier series representation (8.6) that for each $h \in L^{2}(\Omega)$ such that $h$ is $L^{2}$-orthogonal to $N$ there exists a unique $v \in N^{\perp}$ satisfying $\square v=h$. Moreover [8, 9],

$$
\|v\|_{\infty} \leq C\|h\|_{2}
$$

where the constant $C$ is independent of $h$. Since the quotient of the norms of $\sin j x \mathrm{e}^{i k t}$ in $E$ and in $L^{2}(\Omega)$ is $\left|j^{2}-k^{2}\right|$ and since $\left|j^{2}-k^{2}\right| \rightarrow \infty$ as $j^{2}+k^{2} \rightarrow \infty$, $j \neq|k|$, it follows from a standard argument that the embedding $N^{\perp} \hookrightarrow L^{2}(\Omega)$ is 
compact. On the other hand, for each $u \in N,\|u\|=\|u\|_{2}$. So $N$ is not compactly embedded in $L^{2}(\Omega)$.

A function $u$ is said to be a weak solution of (8.1) if $u \in E$ and

$$
\int_{\Omega} u \square \varphi d x d t=\int_{\Omega} f(x, t, u) \varphi d x d t
$$

for all smooth $\varphi \in E$. If $f$ is sufficiently smooth and satisfies (8.3), then weak solutions of (8.1) are known to be classical ones [9, 37].

Let

$$
F(x, t, \xi):=\int_{0}^{\xi} f(x, t, s) d s,
$$

where $f$ satisfies (8.2)-(8.5), and consider the functional

$$
\Phi(u):=\frac{1}{2} \int_{\Omega}\left(u_{t}^{2}-u_{x}^{2}\right) d x d t+\int_{\Omega} F(x, t, u) d x d t .
$$

It is easy to see [38, Appendix B] that $\Phi \in C^{1}(E, \mathbf{R})$ and critical points of $\Phi$ are weak solutions of (8.1). Since the $E$ - and the $L^{2}$-norm coincide on $N$, we cannot expect $\Phi$ to be of class $C^{2}$ even if $f$ is smooth (the second term of the right-hand side of (8.8) is in fact in $C^{2}\left(L^{2}(\Omega), \mathbf{R}\right)$ if and only if $F$ is quadratic with respect to $\xi$, cf. [3, Example 1.4.6]). Therefore Theorem 5.4 and Corollary 5.5 cannot be applied to the study of (8.1).

Let $0<\lambda_{1}<\lambda_{2} \leq \ldots$ be the positive eigenvalues of $\square$ and let $e_{1}, e_{2}, \ldots$ be the corresponding eigenfunctions chosen in such a way that $e_{n}=\sin j x \cos k t$ or $e_{n}=\sin j x \sin k t$ for some $j, k$ with $j^{2}-k^{2}=\lambda_{n}$. Then $\left\langle e_{n}, e_{m}\right\rangle=0$ if $m \neq n$. Define

$$
E_{0}:=\left\{u \in E: u(x, t)=\sum_{k^{2}-j^{2} \geq 0} c_{j k} \sin j x \mathrm{e}^{i k t}\right\}
$$

and

$$
E_{n}:=E_{0} \oplus \operatorname{span}\left\{e_{1}, \ldots, e_{n}\right\} .
$$

Note that the first term on the right-hand side of (8.8) is positive semidefinite on $E_{0}$ and negative definite on $\operatorname{span}\left\{e_{1}, \ldots, e_{n}\right\}$. Clearly, $\left(E_{n}\right)_{n=1}^{\infty}$ is a filtration of $E$. Let $\mathcal{E}:=\left\{E_{n}, n\right\}_{n=1}^{\infty}$. We will show that $\Phi$ satisfies (PS)* under suitable assumptions on $f$. Since $E=N \oplus N^{\perp}$, each $u \in E$ has the representation $u=z+v$, where $z \in N$ and $v \in N^{\perp}$.

Lemma 8.1. (i) For each fixed $v \in N^{\perp}$,

$$
\inf _{z \in N} \int_{\Omega} F(x, t, z+v) d x d t
$$

is attained at a unique $z:=z(v)$.

(ii) $z\left(v_{j}\right) \rightarrow z(\bar{v})$ (in $E$ ) whenever $v_{j} \rightarrow \bar{v}$ in $L^{2}(\Omega)$.

Proof. (i) Since $F(x, t, \xi) \geq \varepsilon \xi^{2} / 2$ according to (8.3),

$$
\int_{\Omega} F(x, t, z+v) d x d t \geq \frac{\varepsilon}{2}\|z+v\|_{2}^{2} .
$$

Hence the functional $z \mapsto \int_{\Omega} F(x, t, z+v) d x d t$ is coercive (recall that $\|z\|=\|z\|_{2}$ ). Since it is also strictly convex, the conclusion follows. 
(ii) Let $v_{j} \rightarrow \bar{v}$ in $L^{2}(\Omega)$ and let $\left(v_{m}\right)$ be a subsequence of $\left(v_{j}\right)$. Since

$$
\int_{\Omega} F\left(x, t, z\left(v_{m}\right)+v_{m}\right) d x d t \leq \int_{\Omega} F\left(x, t, v_{m}\right) d x d t,
$$

it follows from (8.10) that $z\left(v_{m}\right)$ is bounded. We may assume (taking a subsequence if necessary) that $z\left(v_{m}\right) \rightarrow \bar{z}$ weakly. Since $z(v)$ is a critical point of the functional $z \mapsto \int_{\Omega}(F(x, t, z+v) d x d t$,

$$
\int_{\Omega} f(x, t, z(v)+v) \varphi d x d t=0 \quad \text { for all } \varphi \in N .
$$

This and (8.3) imply

$$
\begin{aligned}
\varepsilon\left\|\bar{z}-z\left(v_{m}\right)\right\|_{2}^{2} & \leq \int_{\Omega}\left(f\left(x, t, \bar{z}+v_{m}\right)-f\left(x, t, z\left(v_{m}\right)+v_{m}\right)\right)\left(\bar{z}-z\left(v_{m}\right)\right) d x d t \\
& =\int_{\Omega} f\left(x, t, \bar{z}+v_{m}\right)\left(\bar{z}-z\left(v_{m}\right)\right) d x d t .
\end{aligned}
$$

Since $f\left(x, t, \bar{z}+v_{m}\right) \rightarrow f(x, t, \bar{z}+\bar{v})$ in $L^{2}(\Omega)$ and $z\left(v_{m}\right) \rightarrow \bar{z}$ weakly in $L^{2}(\Omega)$, the second integral above tends to zero. So $z\left(v_{m}\right) \rightarrow \bar{z}$ strongly. Moreover,

$$
\int_{\Omega} F\left(x, t, z(\bar{v})+v_{m}\right) d x d t \geq \int_{\Omega} F\left(x, t, z\left(v_{m}\right)+v_{m}\right) d x d t
$$

according to the minimizing property of $z(v)$. Passing to the limit we obtain

$$
\int_{\Omega} F(x, t, z(\bar{v})+\bar{v}) d x d t \geq \int_{\Omega} F(x, t, \bar{z}+\bar{v}) d x d t .
$$

Hence $\bar{z}=z(\bar{v})$.

We have shown that each subsequence of $\left(z\left(v_{j}\right)\right)$ contains a subsequence converging to $z(\bar{v})$. It follows that $z\left(v_{j}\right) \rightarrow z(\bar{v})$.

A similar result, for superlinear $f$, has been obtained by Tanaka [43, Lemma 1.1].

Denote the spectrum of the operator $\square$ subject to the boundary and the periodicity conditions in (8.1) by $\sigma(\square)$. Let

$$
G(x, t, \xi):=\int_{0}^{\xi} g(x, t, s) d s .
$$

Proposition 8.2. The functional $\Phi$ (given by (8.8)) satisfies $(P S) *$ if either $b \notin$ $\sigma(\square)$ or $g$ is bounded and $G(x, t, \xi) \rightarrow \infty$ (or $G(x, t, \xi) \rightarrow-\infty)$ uniformly in $(x, t)$ as $|\xi| \rightarrow \infty$. Moreover, under these conditions $\left.\Phi\right|_{E_{n}}$ satisfies (PS) for each $n$.

Proof. Let $\left(u_{j}\right)$ be a $(\mathrm{PS})^{*}$-sequence. Since $F(x, t, \xi)=\frac{1}{2} b \xi^{2}+G(x, t, \xi)$,

$$
\Phi(u)=\frac{1}{2} \int_{\Omega}\left(u_{t}^{2}-u_{x}^{2}+b u^{2}\right) d x d t+\int_{\Omega} G(x, t, u) d x d t=: \frac{1}{2}\langle L u, u\rangle+\psi(u) .
$$


Suppose $b \in \sigma(\square)$. Let

$$
\begin{aligned}
& E^{+}:=\left\{u \in E: u(x, t)=\sum_{k^{2}-j^{2}+b>0} c_{j k} \sin j x \mathrm{e}^{i k t}\right\}, \\
& E^{-}:=\left\{u \in E: u(x, t)=\sum_{k^{2}-j^{2}+b<0} c_{j k} \sin j x \mathrm{e}^{i k t}\right\}, \\
& E^{0}:=\left\{u \in E: u(x, t)=\sum_{k^{2}-j^{2}+b=0} c_{j k} \sin j x \mathrm{e}^{i k t}\right\},
\end{aligned}
$$

and write $u=u^{+}+u^{0}+u^{-} \in E^{+} \oplus E^{0} \oplus E^{-}$. Note that $L\left(E^{ \pm}\right) \subset E^{ \pm}$and $L\left(E_{n}\right) \subset E_{n}$. Since the quadratic form $u \mapsto\langle L u, u\rangle$ is positive definite on $E^{+}$, negative definite on $E^{-}$and since

$$
P_{n_{j}} \nabla \Phi\left(u_{j}\right)=L u_{j}^{+}+L u_{j}^{-}+P_{n_{j}} \nabla \psi\left(u_{j}\right) \rightarrow 0
$$

and $\nabla \psi(E)$ is bounded (because $g$ is), it follows that the sequence $\left(u_{j}^{+}+u_{j}^{-}\right)$is bounded, and so is $\psi\left(u_{j}\right)$. By the mean value theorem,

$$
\left|\psi\left(u_{j}^{0}\right)-\psi\left(u_{j}\right)\right| \leq \sup _{u \in E}\|\nabla \psi(u)\|\left\|u_{j}^{+}+u_{j}^{-}\right\| .
$$

Hence

$$
\psi\left(u_{j}^{0}\right)=\int_{\Omega} G\left(x, t, u_{j}^{0}\right) d x d t
$$

is bounded. Since $E^{0}$ is finite dimensional and $G(x, t, \xi) \rightarrow \infty$ (or $\left.-\infty\right)$ as $|\xi| \rightarrow \infty$, the sequence $\left(u_{j}^{0}\right)$, and therefore also $\left(u_{j}\right)$, is bounded.

Let $u_{j}=z_{j}+v_{j}$, where $z_{j} \in N$ and $v_{j} \in N^{\perp}$. We may assume after passing to a subsequence that $v_{j} \rightarrow \bar{v}$ weakly in $N^{\perp}$ and strongly in $L^{2}(\Omega)$. Since $P_{n_{j}} \nabla \Phi\left(u_{j}\right) \rightarrow$ 0 and $N \subset E_{n_{j}}$ (cf. (8.9)),

$$
\int_{\Omega} f\left(x, t, z_{j}+v_{j}\right)\left(z_{j}-z\left(v_{j}\right)\right) d x d t=\left\langle\nabla \Phi\left(u_{j}\right), z_{j}-z\left(v_{j}\right)\right\rangle \rightarrow 0 .
$$

By (8.3) and (8.11),

$$
\begin{aligned}
\varepsilon\left\|z_{j}-z\left(v_{j}\right)\right\|_{2}^{2} & \leq \int_{\Omega}\left(f\left(x, t, z_{j}+v_{j}\right)-f\left(x, t, z\left(v_{j}\right)+v_{j}\right)\right)\left(z_{j}-z\left(v_{j}\right)\right) d x d t \\
& =\int_{\Omega} f\left(x, t, z_{j}+v_{j}\right)\left(z_{j}-z\left(v_{j}\right)\right) d x d t \rightarrow 0 .
\end{aligned}
$$

So $z_{j}-z\left(v_{j}\right) \rightarrow 0$ in $E$. Since $z\left(v_{j}\right) \rightarrow z(\bar{v})$ according to Lemma 8.1, $z_{j} \rightarrow z(\bar{v})$ in $E$. Consequently, $u_{j}=z_{j}+v_{j} \rightarrow z(\bar{v})+\bar{v}$ in $L^{2}(\Omega)$ and therefore $\nabla \psi\left(u_{j}\right) \rightarrow$ $\nabla \psi(z(\bar{v})+\bar{v})$. Since $L$ is invertible on $E^{+} \oplus E^{-},(8.13)$ shows that $u_{j} \rightarrow z(\bar{v})+\bar{v}$ in $E$.

Suppose now that $b \notin \sigma(\square)$. Then $E^{0}=\{0\}$. By (8.4), for each $\varepsilon^{\prime}>0$ there is a constant $C=C\left(\varepsilon^{\prime}\right)$ such that $|g(x, t, \xi)| \leq \varepsilon^{\prime}|\xi|+C\left(\varepsilon^{\prime}\right)$. Hence $\nabla \psi(u)=o(\|u\|)$ as $\|u\| \rightarrow \infty$ (cf. (7.8) and the following lines). Since $L$ is now invertible, it follows from $(8.13)$ that $\left(u_{j}\right)$ is a bounded sequence. The remaining part of the proof is the same as above.

Finally, since $N \subset E_{n}$, an obvious modification of the above argument shows that $\left.\Phi\right|_{E_{n}}$ satisfies (PS).

Let

$$
G_{0}(x, t, \xi):=\int_{0}^{\xi} g_{0}(x, t, s) d s
$$


and

$$
\Phi(u)=\frac{1}{2} \int_{\Omega}\left(u_{t}^{2}-u_{x}^{2}+b_{0} u^{2}\right) d x d t+\int_{\Omega} G_{0}(x, t, u) d x d t=: \frac{1}{2}\left\langle L_{0} u, u\right\rangle+\psi_{0}(u) .
$$

Then

$$
\begin{aligned}
F^{+} & :=\left\{u \in E: u(x, t)=\sum_{k^{2}-j^{2}+b_{0}>0} c_{j k} \sin j x \mathrm{e}^{i k t}\right\} \\
F^{-} & :=\left\{u \in E: u(x, t)=\sum_{k^{2}-j^{2}+b_{0}<0} c_{j k} \sin j x \mathrm{e}^{i k t}\right\}, \\
F^{0} & :=\left\{u \in E: u(x, t)=\sum_{k^{2}-j^{2}+b_{0}=0} c_{j k} \sin j x \mathrm{e}^{i k t}\right\}
\end{aligned}
$$

are the subspaces on which $\left\langle L_{0} u, u\right\rangle$ is positive definite, negative definite and zero.

Proposition 8.3. $\Phi$ satisfies the local linking condition at 0 in each of the following cases:

(i) $b_{0} \notin \sigma(\square)$;

(ii) $b_{0} \in \sigma(\square)$ and there is a $\delta>0$ such that $G_{0}(x, t, \xi) \geq 0$ whenever $|\xi| \leq \delta$;

(iii) $b_{0} \in \sigma(\square)$ and there is a $\delta>0$ such that $G_{0}(x, t, \xi) \leq 0$ whenever $|\xi| \leq \delta$.

Moreover, $c_{\mathcal{E}}^{-q_{0}}(\Phi, 0) \neq[0]$, where $q_{0}$ is the number of eigenvalues of $\square$ in the interval $\left(0, b_{0}\right]$ (counted with their multiplicity) if (i) or (ii) is satisfied, and in the interval $\left(0, b_{0}\right)$ if (iii) holds.

Proof. Let $E=Y \oplus Z$. If (i) is satisfied, we take $Y=F^{-}$and $Z=F^{+}$, if (ii) holds, $Y=F^{-}$and $Z=F^{+} \oplus F^{0}$, and in the remaining case $Y=F^{0} \oplus F^{-}$and $Z=F^{+}$. We will show that $\Phi \leq 0$ on $Y \cap B(0, \rho)$ and $\Phi \geq 0$ on $Z \cap B(0, \rho)$ if $\rho$ is small enough. Assuming this, it is easy to obtain the second conclusion. Indeed, suppose that (i) or (ii) is satisfied. Then $Y=F^{-}$. Since $E_{n}$ is the subspace of $E$ obtained by taking the sums in (8.6) over all $j, k$ with $j^{2}-k^{2} \leq \lambda_{n}$ and $Y$ is obtained by summing over $j, k$ with $j^{2}-k^{2}>b_{0}, Y \cap E_{n}$ is spanned by the eigenfunctions $e_{m}$ such that $b_{0}<\lambda_{m} \leq \lambda_{n}$. Therefore $\operatorname{dim}\left(Y \cap E_{n}\right)=n-q_{0}$ for all $n>q_{0}$. If (iii) is satisfied, then $Y=F^{0} \oplus F^{-}$and $Y \cap E_{n}$ is spanned by all $e_{m}$ such that $b_{0} \leq \lambda_{m} \leq \lambda_{n}$. So again $\operatorname{dim}\left(Y \cap E_{n}\right)=n-q_{0}$ for $n>q_{0}$. Since obviously $E_{n}=\left(Y \cap E_{n}\right) \oplus\left(Z \cap E_{n}\right), c_{\mathcal{E}}^{-q_{0}}(\Phi, 0) \neq[0]$ according to Theorem 5.6.

We verify the local linking condition only in case (iii) (and make comments on other cases when suitable). Let $u \in Z \equiv F^{+}$and write $u=v+z, v \in N^{\perp} \cap F^{+}$, $z \in N$. First we show that there is an $r>0$ such that if $D_{r}:=\left\{u \in Z:\|u\|_{2} \leq r\right\}$, then $\inf _{D_{r}} \Phi \geq 0$. Let $\left(u_{j}\right) \subset D_{r}$ be a sequence such that $\Phi\left(u_{j}\right)$ tends to the infimum. Since the quadratic form in (8.14) is positive definite on $F^{+}$and $\psi_{0}$ is bounded on $D_{r}, \Phi\left(u_{j}\right) \rightarrow \infty$ if $\left\|u_{j}\right\| \rightarrow \infty$ in $E$. Hence $\left(u_{j}\right)$ is bounded in $E$ and we may assume taking a subsequence that $u_{j} \rightarrow \widetilde{u}$ weakly in $E$ (and $v_{j} \rightarrow \widetilde{v}$ strongly in $L^{2}(\Omega)$ ). Furthermore, the function $\xi \mapsto F(x, t, \xi)$ is convex and the quadratic form in (8.8) is positive semidefinite except on the finite dimensional subspace of $F^{+}$on which $-b_{0}<k^{2}-j^{2}<0$. Therefore $\Phi$ is weakly lower semicontinuous on $F^{+}$and $\Phi(\widetilde{u})=\inf _{D_{r}} \Phi$ (in particular, the infimum is a finite number). It follows that

$$
\langle\nabla \Phi(\widetilde{u}), \varphi\rangle=\lambda \int_{\Omega} \widetilde{u} \varphi d x d t
$$


for some $\lambda \leq 0$ and all $\varphi \in F^{+}$( $\lambda$ is a Lagrange multiplier which takes into account the fact that $\widetilde{u}$ may be on the boundary of $D_{r}$ ). Choosing $\varphi=\widetilde{u}$, we obtain

$$
\lambda\|\widetilde{u}\|_{2}^{2}=\int_{\Omega}\left(\widetilde{u}_{t}^{2}-\widetilde{u}_{x}^{2}\right) d x d t+\int_{\Omega} f(x, t, \widetilde{u}) \widetilde{u} d x d t .
$$

Below $c_{1}, c_{2}, \ldots$ will denote different positive constants. Recall that the quadratic form above is negative definite on the finite dimensional subspace on which $-b_{0}<$ $k^{2}-j^{2}<0$ and positive semidefinite otherwise. Thus $\int_{\Omega}\left(\widetilde{u}_{t}^{2}-\widetilde{u}_{x}^{2}\right) d x d t \geq-c_{1}\|\widetilde{u}\|_{2}^{2}$. Since $f(x, t, \xi) \xi \geq 0$ for all $x, t, \xi$, we have

$$
\lambda\|\widetilde{u}\|_{2}^{2} \geq-c_{1}\|\widetilde{u}\|_{2}^{2}
$$

and $|\lambda| \leq c_{1}$ (if $\widetilde{u}=0$, then $\lambda=0$ because $\widetilde{u} \notin \partial D_{r}$ ). It follows from (8.15) that

$$
\square \widetilde{v}=P f(x, t, \widetilde{u})-\lambda \widetilde{u} \equiv P(f(x, t, \widetilde{u})-\lambda \widetilde{u})
$$

in the sense of distributions, where

$$
P\left(\sum_{j, k} d_{j k} \sin j x \mathrm{e}^{i k t}\right):=\sum_{k^{2}-j^{2}+b_{0}>0} d_{j k} \sin j x \mathrm{e}^{i k t}
$$

is a bounded projector in $L^{2}(\Omega)$. Employing (8.7) and (8.4), (8.5) we obtain

$$
\|\widetilde{v}\|_{\infty} \leq c_{2}\|P(f(x, t, \widetilde{u})-\lambda \widetilde{u})\|_{2} \leq c_{3}\|\widetilde{u}\|_{2}
$$

It has been shown in [27, Lemma 4.3], cf. also [37, Lemma 3.7], that if a function $h$ satisfies (8.3) and

$$
\square v=P h(x, t, u),
$$

where $u=v+z$, then $\|z\|_{\infty} \leq c_{4}\|v\|_{\infty}$ (in [27] the setup is slightly different but our conclusion here remains true with the same proof). Since $\lambda \leq 0, f(x, t, \xi)-\lambda \xi$ satisfies (8.3). Consequently, $\|\widetilde{u}\|_{\infty} \leq c_{5}\|\widetilde{u}\|_{2}$. It follows therefore from (8.5) that

$$
\Phi(\widetilde{u})=\frac{1}{2}\left\langle L_{0} \widetilde{u}, \widetilde{u}\right\rangle+\int_{\Omega} G_{0}(x, t, \widetilde{u}) d x d t \geq 0
$$

whenever $r$ is sufficiently small. Note that in case (ii) $Z=F^{+} \oplus F^{0}$, the quadratic form above is positive semidefinite on $Z$, and $G_{0}(x, t, \widetilde{u}) \geq 0$ if $\|\widetilde{u}\|_{\infty} \leq c_{5} r \leq \delta$. Hence (8.17) still holds.

We have shown that $\inf _{D_{r}} \Phi \geq 0$. Since the embedding $E \hookrightarrow L^{2}(\Omega)$ is continuous, $B(0, \rho) \subset D_{r}$ for some $\rho$ and $\Phi \geq 0$ on $Z \cap B(0, \rho)$.

Now let $u=u^{0}+u^{-} \in F^{0} \oplus F^{-} \equiv Y$. It suffices to show that there is an $r>0$ such that $\sup _{C_{r}} \Phi \leq 0$, where $C_{r}:=\left\{u \in Y:\|u\|_{2} \leq r\right\}$ (note that cases (i) and (ii) are simpler because $F^{0}=\{0\}$ ). Let $\left(u_{j}\right) \subset C_{r}$ be a maximizing sequence for $\Phi$. Since

$$
\Phi(u)=\frac{1}{2}\left\langle L_{0} u^{-}, u^{-}\right\rangle+\int_{\Omega} G_{0}(x, t, u) d x d t,
$$

the first term on the right-hand side above is negative definite on $F^{-}$and the second one is bounded on $C_{r}$, it follows that $\Phi\left(u_{j}\right) \rightarrow-\infty$ as $\left\|u_{j}^{-}\right\| \rightarrow \infty$ in $E$. So we may assume that $u_{j} \rightarrow \widetilde{u}$ weakly in $E$ and strongly in $L^{2}(\Omega)$ (we have used that $Y \subset N^{\perp}$ and the embedding $N^{\perp} \hookrightarrow L^{2}(\Omega)$ is compact). Since negative definite quadratic forms are weakly upper semicontinuous, $\lim _{j \rightarrow \infty} \Phi\left(u_{j}\right) \leq \Phi(\widetilde{u})$. So $\Phi(\widetilde{u})=\sup _{C_{r}} \Phi$ and

$$
\square \widetilde{u}=Q f(x, t, \widetilde{u})-\lambda \widetilde{u} \equiv Q(f(x, t, \widetilde{u})-\lambda \widetilde{u})
$$


in the sense of distributions, where $\lambda \geq 0$ and $Q$ is the projector onto the subspace of $L^{2}(\Omega)$ on which $k^{2}-j^{2}+b_{0} \leq 0$. The quadratic form on the right-hand side of (8.16) is now negative definite, so

$$
0 \leq \lambda\|\widetilde{u}\|_{2}^{2} \leq \int_{\Omega} f(x, t, \widetilde{u}) \widetilde{u} d x d t \leq c_{6}\|\widetilde{u}\|_{2}^{2}
$$

and $0 \leq \lambda \leq c_{6}$. By (8.7) and (8.19), $\|\widetilde{u}\|_{\infty} \leq c_{7}\|\widetilde{u}\|_{2}$. If $r$ is small enough, then $\|\widetilde{u}\|_{\infty} \leq \delta$, and it follows from (8.18) that $\Phi(\widetilde{u}) \leq 0$.

Theorem 8.4. Suppose that $f$ satisfies (8.2)-(8.5) and either $b \notin \sigma(\square)$ or $g$ is bounded and $G(x, t, \xi) \rightarrow \infty$ uniformly in $(x, t)$ as $|\xi| \rightarrow \infty$. Then the wave equation (8.1) has a nontrivial weak solution in each of the following cases:

(i) $b_{0} \notin \sigma(\square)$ and $\left(0, b_{0}\right] \cap \sigma(\square) \neq(0, b] \cap \sigma(\square)$;

(ii) $b_{0} \in \sigma(\square),\left(0, b_{0}\right] \cap \sigma(\square) \neq(0, b] \cap \sigma(\square)$ and there is a $\delta>0$ such that $G_{0}(x, t, \xi) \geq 0$ whenever $|\xi| \leq \delta$;

(iii) $b_{0} \in \sigma(\square),\left(0, b_{0}\right) \cap \sigma(\square) \neq(0, b] \cap \sigma(\square)$ and there is a $\delta>0$ such that $G_{0}(x, t, \xi) \leq 0$ whenever $|\xi| \leq \delta$.

Proof. Suppose that 0 is the only critical point of $\Phi$. If $b \notin \sigma(\square)$, let $\Phi_{\lambda}(u):=$ $\frac{1}{2}\langle L u, u\rangle+(1-\lambda) \psi(u)($ cf. (8.12)). Since $\nabla \psi(u)=o(\|u\|)$ as $\|u\| \rightarrow \infty$, it follows from Propositions 2.12 and 2.14 that $c_{\mathcal{E}}^{*}(\Phi, 0)=c_{\mathcal{E}}^{*}(\Phi, K(\Phi))=c_{\mathcal{E}}^{*}\left(\Phi_{1}, 0\right)$ (cf. the proof of Theorem 7.4). Since $E^{-} \cap E_{n}$ is spanned by the eigenfunctions $e_{m}$ such that $b<\lambda_{m} \leq \lambda_{n}$, we see that $\operatorname{dim}\left(E^{-} \cap E_{n}\right)=n-q_{\infty}$ if $n>q_{\infty}$, where $q_{\infty}$ is the number of eigenvalues of $\square$ in the interval $(0, b]$ (counted with their multiplicity). Hence $M_{\mathcal{E}}^{-}(L)=-q_{\infty}$ and

$$
c_{\mathcal{E}}^{q}(\Phi, 0)=c_{\mathcal{E}}^{q}(\Phi, K(\Phi))= \begin{cases}{[\mathcal{F}]} & \text { if } q=-q_{\infty} \\ {[0]} & \text { otherwise }\end{cases}
$$

Now let $b \in \sigma(\square)$. Since $\left.\Phi\right|_{E_{n}}$ satisfies (PS), we may proceed as in the proof of Lemma 7.6(i) and we obtain (8.20) again (note that here $N(L) \subset E_{n}$ for almost all $n$, so $P_{n} N(L)=N(L)$ and $\left.E_{n}^{\prime}=E_{n}\right)$. According to Proposition $8.3, c_{\mathcal{E}}^{-q_{0}}(\Phi, 0) \neq$ [0]. So $q_{0}=q_{\infty}$. On the other hand, it follows from the definitions of $q_{0}$ and $q_{\infty}$ and from our hypotheses on the intersection with $\sigma(\square)$ that $q_{0} \neq q_{\infty}$.

Theorem 8.5. Suppose that $f$ satisfies (8.2)-(8.5) and either $b \notin \sigma(\square)$ or $g$ is bounded and $G(x, t, \xi) \rightarrow-\infty$ uniformly in $(x, t)$ as $|\xi| \rightarrow \infty$. Then the wave equation (8.1) has a nontrivial weak solution if the interval $(0, b]$ in the assumptions (i)-(iii) of Theorem 8.4 is replaced by $(0, b)$.

Proof. The only difference compared to the proof of the preceding theorem is that if $b \in \sigma(\square)$, we now use the argument of Lemma 7.6(ii) and obtain $c_{\mathcal{E}}^{q}(\Phi, 0)=[\mathcal{F}]$ for $q=M_{\mathcal{E}}^{-}(L)+M^{0}(L)$ and [0] otherwise. So (8.20) holds with $q_{\infty}=-M_{\mathcal{E}}^{-}(L)-$ $M^{0}(L)$, and it follows that in the present case $q_{\infty}$ is the number of eigenvalues of $\square$ in the interval $(0, b)$.

Remark 8.6. If $b \notin \sigma(\square)$, then (8.4) may be replaced by the slightly weaker hypothesis that $|g(x, t, \xi)| \leq \alpha|\xi|+\beta$, where $\alpha$ is less than the distance from $b$ to $\sigma(\square)$ [27, Corollary 5.2].

Remark 8.7. Theorems 8.4 and 8.5 extend some results of $[1,27]$. In [1] it was assumed that $f \in C^{1}$, the derivative $f_{\xi}$ is bounded, bounded away from zero and $b \notin \sigma(\square)$ (on the other hand, if $b_{0} \in \sigma(\square)$, our hypothesis at $\xi=0$ is different-and 
rather more restrictive - than the corresponding one in [1]). Theorem 8.4 is a slight generalization of the main result of [27] (in [27] the sign conditions (ii) and (iii) are for $g_{0}(x, t, \xi) \xi$; here they are for $\left.G_{0}(x, t, \xi)\right)$. Theorem 8.5 is new.

\section{Elliptic SYSTEM}

Let $\Omega \subset \mathbf{R}^{N}$ be a bounded domain with smooth boundary, let $F \in C^{1}\left(\bar{\Omega} \times \mathbf{R}^{2}, \mathbf{R}\right)$ and consider the Dirichlet problem

$$
\begin{cases}-\Delta u=F_{v}(x, u, v) & \text { in } \Omega, \\ -\Delta v=F_{u}(x, u, v) & \text { in } \Omega, \\ u=v=0 & \text { on } \partial \Omega .\end{cases}
$$

Problems of this type have been studied recently in [14] for subquadratic $F$, in $[16,23]$ for superquadratic $F$, and in [42] a bifurcation problem for (9.1) has been considered. Here we assume that $F$ is asymptotically quadratic, or more precisely, that

$$
\begin{aligned}
F(x, u, v) & =\frac{1}{2} a u^{2}+b u v+\frac{1}{2} c v^{2}+G(x, u, v), \text { where }\left|G_{u}(x, u, v)\right|+\left|G_{v}(x, u, v)\right| \\
& =o(|u|+|v|) \text { uniformly in } x \text { as }|u|+|v| \rightarrow \infty
\end{aligned}
$$

and

$$
\begin{aligned}
F(x, u, v) & =\frac{1}{2} a_{0} u^{2}+b_{0} u v+\frac{1}{2} c_{0} v^{2}+G_{0}(x, u, v), \\
& \quad \text { where }\left|\left(G_{0}\right)_{u}(x, u, v)\right|+\left|\left(G_{0}\right)_{v}(x, u, v)\right| \\
& =o(|u|+|v|) \text { uniformly in } x \text { as }|u|+|v| \rightarrow 0 .
\end{aligned}
$$

For simplicity $a, b, c$ and $a_{0}, b_{0}, c_{0}$ are assumed to be constant, though $x$-dependence could be admitted.

Let $H_{0}^{1}(\Omega)$ be the usual Sobolev space (of real-valued functions) and set $E:=$ $H_{0}^{1}(\Omega) \times H_{0}^{1}(\Omega)$. Then $E$ is a Hilbert space with inner product given by

$$
\left\langle(u, v),\left(u^{\prime}, v^{\prime}\right)\right\rangle:=\int_{\Omega}\left(\nabla u \cdot \nabla u^{\prime}+\nabla v \cdot \nabla v^{\prime}\right) d x .
$$

It is easily seen from [38, Appendix B] and (9.2) that the functional $\Phi: E \rightarrow \mathbf{R}$ defined by

$$
\Phi(u, v):=\int_{\Omega} \nabla u \cdot \nabla v d x-\int_{\Omega} F(x, u, v) d x
$$

is of class $C^{1}$ and critical points of $\Phi$ correspond to weak solutions of (9.1). Moreover, weak solutions are classical ones if either $N=1$ or $N \geq 2$ and $F_{u}, F_{v}$ are locally Hölder continuous.

Let $0<\lambda_{1}<\lambda_{2} \leq \lambda_{3} \leq \ldots$ be the eigenvalues of $-\Delta$ in $H_{0}^{1}(\Omega)$ and let $\left(e_{n}\right)_{n=1}^{\infty}$ be the corresponding orthonormal basis of eigenfunctions. Define

$$
F_{n}:=\operatorname{span}\left\{\left(e_{n}, 0\right),\left(0, e_{n}\right)\right\}, \quad E_{n}:=\operatorname{span}\left\{\left(e_{i}, 0\right),\left(0, e_{j}\right): 1 \leq i, j \leq n\right\}
$$


and $\mathcal{E}:=\left\{E_{n}, n\right\}_{n=1}^{\infty}$. Furthermore, set

$$
\begin{aligned}
\Phi(u, v) & =\int_{\Omega}\left(\nabla u \cdot \nabla v-\frac{1}{2} a u^{2}-b u v-\frac{1}{2} c v^{2}\right) d x-\int_{\Omega} G(x, u, v) d x \\
& =: \frac{1}{2}\langle L(u, v),(u, v)\rangle-\varphi(u, v)
\end{aligned}
$$

and

$$
\begin{aligned}
\Phi(u, v) & =\int_{\Omega}\left(\nabla u \cdot \nabla v-\frac{1}{2} a_{0} u^{2}-b_{0} u v-\frac{1}{2} c_{0} v^{2}\right) d x-\int_{\Omega} G_{0}(x, u, v) d x \\
& =: \frac{1}{2}\left\langle L_{0}(u, v),(u, v)\right\rangle-\varphi_{0}(u, v) .
\end{aligned}
$$

Let

$$
A:=\left(\begin{array}{ll}
a & b \\
b & c
\end{array}\right)
$$

be the matrix representing the quadratic form in (9.2). Since $\left(e_{n}\right)_{n=1}^{\infty}$ is an orthonormal basis of eigenfunctions, $\int_{\Omega} e_{m} e_{n} d x=0$ if $m \neq n$ and $1=\int_{\Omega}\left|\nabla e_{n}\right|^{2} d x=$ $\lambda_{n} \int_{\Omega} e_{n}^{2} d x$. Using this it is easy to see that $L\left(F_{n}\right) \subset F_{n}, L\left(E_{n}\right) \subset E_{n}$ and the same is true for $L_{0}$. Moreover, if $(u, v)=\left(\alpha e_{n}, \beta e_{n}\right) \in F_{n}$, then

$$
\frac{1}{2}\langle L(u, v),(u, v)\rangle=\alpha \beta-\frac{1}{\lambda_{n}}\left(\frac{1}{2} a \alpha^{2}+b \alpha \beta+\frac{1}{2} c \beta^{2}\right) .
$$

Hence the linear mapping $\left.L\right|_{F_{n}}: F_{n} \rightarrow F_{n}$ is represented by the matrix

$$
T_{n}(A):=\left(\begin{array}{cc}
-\frac{a}{\lambda_{n}} & 1-\frac{b}{\lambda_{n}} \\
1-\frac{b}{\lambda_{n}} & -\frac{c}{\lambda_{n}}
\end{array}\right) .
$$

Let

$$
\begin{aligned}
i^{-}(A) & :=\sum_{n=1}^{\infty}\left(M^{-}\left(T_{n}(A)\right)-1\right) \\
i^{+}(A) & :=\sum_{n=1}^{\infty}\left(M^{+}\left(T_{n}(A)\right)-1\right)
\end{aligned}
$$

and

$$
i^{0}(A):=\sum_{n=1}^{\infty} M^{0}\left(T_{n}(A)\right)
$$

Since $\lambda_{n} \rightarrow \infty$ as $n \rightarrow \infty, i^{ \pm}(A)$ and $i^{0}(A)$ are well-defined and finite. Moreover, $N(L) \subset E_{n}$ for some $n, \operatorname{dim} N(L)=i^{0}(A), Q_{n} L=L$ (because $L\left(E_{n}\right) \subset E_{n}$ ) and $i^{-}(A)=M_{\mathcal{E}}^{-}(L)$. It is also easy to see that $i^{-}(A)+i^{+}(A)+i^{0}(A)=0$.

Lemma 9.1. Suppose that $F$ satisfies (9.2). Then $\Phi$ satisfies (PS)* in each of the following two cases:

(i) $i^{0}(A)=0$;

(ii) $G_{u}, G_{v}$ are bounded and $G(x, u, v) \rightarrow \infty(\operatorname{or} G(x, u, v) \rightarrow-\infty)$ uniformly in $x$ as $|u|+|v| \rightarrow \infty$.

Moreover, under the above assumptions $\left.\Phi\right|_{E_{n}}$ satisfies (PS) for each $n$.

The proof uses the same argument as that of Lemma 7.3 and is therefore omitted (note that $\nabla \varphi$ is compact according to [38, Appendix B]). 
Let $F \in C^{2}\left(\bar{\Omega} \times \mathbf{R}^{2}, \mathbf{R}\right)$ and denote the Hessian of $F$ with respect to $u, v$ by $D^{2}$. Suppose that there is a constant $C$ such that

$$
\left\|D^{2} F(x, u, v)\right\| \leq C(1+|u|+|v|)^{p-1},
$$

where $1 \leq p<(N+2) /(N-2)$ if $N>2$ and $1 \leq p<\infty$ if $N=2$ (if $N=1$, no growth restriction (9.4) is necessary). Then $\Phi \in C^{2}(E, \mathbf{R})$ [38, Appendix B].

Denote by $A_{0}$ the matrix which represents the quadratic form in (9.3). Below we formulate two sufficient conditions for the existence of a nontrivial solution to (9.1). The proofs are omitted because they are obtained by an easy modification of the arguments in Theorems 7.4 and 7.5 (here in fact the situation is somewhat simpler: since $P_{n} N(L) \subset N(L)$ for some $n$, it is not necessary to introduce a modified filtration $\mathcal{E}^{\prime}$ as in Theorem 7.5).

Theorem 9.2. Suppose that $F$ satisfies (9.2) and (9.3). If $i^{0}(A)=i^{0}\left(A_{0}\right)=0$ and $i^{-}(A) \neq i^{-}\left(A_{0}\right)$, then (9.1) has a nontrivial weak solution.

Theorem 9.3. Suppose that $F \in C^{2}\left(\bar{\Omega} \times \mathbf{R}^{2}, \mathbf{R}\right)$ satisfies (9.2), (9.3) and (9.4) ((9.4) may be omitted if $N=1)$. If $G_{u}, G_{v}$ are bounded, then (9.1) has a nontrivial weak solution in each of the following two cases:

(i) $G(x, u, v) \rightarrow-\infty$ uniformly in $x$ as $|u|+|v| \rightarrow \infty$ and

$$
i^{-}(A) \notin\left[i^{-}\left(A_{0}\right), i^{-}\left(A_{0}\right)+i^{0}\left(A_{0}\right)\right] ;
$$

(ii) $G(x, u, v) \rightarrow \infty$ uniformly in $x$ as $|u|+|v| \rightarrow \infty$ and

$$
i^{+}(A) \notin\left[i^{+}\left(A_{0}\right), i^{+}\left(A_{0}\right)+i^{0}\left(A_{0}\right)\right] .
$$

If $i^{0}\left(A_{0}\right)=0$, it is not necessary to assume that $F \in C^{2}$, and if $i^{0}(A)=0$, $G_{u}, G_{v}$ need not be bounded and $G$ need not tend to infinity.

Also Theorem 7.10 has a counterpart here. We leave the formulation to the reader and observe only that the local linking condition may be verified e.g. by adapting the argument of $[29$, Theorem 4$]$.

Suppose that $N=1, \Omega=(a, b)$ and $F \in C^{2}\left([a, b] \times \mathbf{R}^{2}, \mathbf{R}\right)$. If $\left(u_{0}, v_{0}\right)$ is a solution of (9.1), then $(u, v) \in E$ is in the nullspace of $\Phi^{\prime \prime}\left(u_{0}, v_{0}\right)$ if and only if

$$
\left\{\begin{array}{l}
-u^{\prime \prime}=F_{u v}\left(x, u_{0}(x), v_{0}(x)\right) u+F_{v v}\left(x, u_{0}(x), v_{0}(x)\right) v \\
-v^{\prime \prime}=F_{u u}\left(x, u_{0}(x), v_{0}(x)\right) u+F_{u v}\left(x, u_{0}(x), v_{0}(x)\right) v \\
u(a)=v(a)=u(b)=v(b)=0
\end{array}\right.
$$

It is well-known that such systems can have at most two linearly independent solutions, so $\operatorname{dim} N\left(\Phi^{\prime \prime}\left(u_{0}, v_{0}\right)\right) \leq 2$ (this is no longer true if $N \geq 2$ ). Using the argument of Theorem 7.8 , we therefore obtain

Theorem 9.4. Suppose that $N=1, F \in C^{2}\left([a, b] \times \mathbf{R}^{2}, \mathbf{R}\right)$ satisfies (9.2), (9.3) and $i^{0}\left(A_{0}\right)=0$. Then (9.1) has at least two nontrivial solutions in each of the following cases:

(i) $\left|i^{-}(A)-i^{-}\left(A_{0}\right)\right| \geq 2$ and $i^{0}(A)=0$;

(ii) $\left|i^{-}(A)-i^{-}\left(A_{0}\right)\right| \geq 2, G_{u}, G_{v}$ are bounded and $G(x, u, v) \rightarrow-\infty$ uniformly in $x$ as $|u|+|v| \rightarrow \infty$;

(iii) $\left|i^{+}(A)-i^{+}\left(A_{0}\right)\right| \geq 2, G_{u}, G_{v}$ are bounded and $G(x, u, v) \rightarrow \infty$ uniformly in $x$ as $|u|+|v| \rightarrow \infty$. 
Remark 9.5. Similar results remain valid for the Neumann problem

$$
\begin{cases}-\Delta u=F_{v}(x, u, v) & \text { in } \Omega, \\ -\Delta v=F_{u}(x, u, v) & \text { in } \Omega, \\ \partial u / \partial n=\partial v / \partial n=0 & \text { on } \partial \Omega .\end{cases}
$$

The appropriate choice of the space is then $E=H^{1}(\Omega) \times H^{1}(\Omega)$ (the inner product and the indices $i^{ \pm}(A), i^{0}(A)$ need to be modified in a rather obvious way). Since the null space of the quadratic form $(u, v) \mapsto \int_{\Omega} \nabla u \cdot \nabla v d x$ in $H^{1}(\Omega) \times H^{1}(\Omega)$ consists of constant functions, it is easy to see that also results analogous to Corollary 7.9 (for $N=1$ ) and Theorem 7.10 (for $N \geq 1$ ) are true here (in the latter case we assume $F$ is periodic in one or both variables $u, v)$.

\section{REFERENCES}

[1] H. Amann and E. Zehnder, Nontrivial solutions for a class of nonresonance problems and applications to nonlinear differential equations, Ann. Scuola Norm. Sup. Pisa (4) 7 (1980), 539-603. MR 82b:47077

[2] _ Periodic solutions of asymptotically linear Hamiltonian systems, Manuscr. Math. 32 (1980), 149-189. MR 82i:58026

[3] J.P. Aubin and I. Ekeland, Applied Nonlinear Analysis, Wiley, New York, $1984 . \quad$ MR 87a:58002

[4] A. Bahri and H. Berestycki, Existence of forced oscillations for some nonlinear differential equations, Comm. Pure Appl. Math. 37 (1984), 403-442. MR 85m:34061

[5] _ Forced vibrations of superquadratic Hamiltonian systems, Acta Math. 152 (1984), 143-197. MR 85g:34041

[6] V. Benci, A new approach to the Morse-Conley theory and some applications, Ann. Mat. Pura Appl. (4) 158 (1991), 231-305. MR 92k:58043

[7] M.L. Bertotti, Forced oscillations of asymptotically linear Hamiltonian systems, Boll. Un. Mat. Ital. (7) 1-B (1987), 729-740. MR 89b:58176

[8] H. Brézis, J.M. Coron and L. Nirenberg, Free vibrations for a nonlinear wave equation and a theorem of P. Rabinowitz, Comm. Pure Appl. Math. 33 (1980), 667-689. MR 81k:35013

[9] H. Brézis and L. Nirenberg, Forced vibrations for a nonlinear wave equation, Comm. Pure Appl. Math. 31 (1978), 1-30. MR 81i:35112

[10] K.C. Chang, Infinite Dimensional Morse Theory and Multiple Solution Problem, Birkhäuser, Boston, 1993. MR 94e:58023

[11] C.C. Conley, Isolated Invariant Sets and the Morse Index, CBMS Regional Conf. Ser. Math., no. 38, Amer. Math. Soc., Providence, R.I., 1978. MR 80c:58009

[12] C.C. Conley and E. Zehnder, The Birkhoff-Lewis fixed point theorem and a conjecture of V.I. Arnold, Invent. Math. 73 (1983), 33-49. MR 85e:58044

[13] _ Morse type index theory for flows and periodic solutions for Hamiltonian equations, Comm. Pure Appl. Math. 37 (1984), 207-253. MR 89b:58021

[14] D.G. Costa and C.A. Magalhães, A variational approach to subquadratic perturbations of elliptic systems, J. Diff. Eq. 111 (1994), 103-122. MR 95f:35082

[15] E.N. Dancer, Degenerate critical points, homotopy indices and Morse inequalities, J. Reine Angew. Math. 350 (1984), 1-22. MR 85i:58033

[16] D.G. de Figueiredo and P. Felmer, On superquadratic elliptic systems, Trans. Amer. Math. Soc. 343 (1994), 99-116. MR 94g:35072

[17] A. Dold, Lectures on Algebraic Topology, Springer-Verlag, Berlin, 1972. MR 54:3685

[18] G. Fournier, D. Lupo, M. Ramos and M. Willem, Limit relative category and critical point theory. In: Dynamics Reported 3 (New Series), C.K.R.T. Jones, U. Kirchgraber and H.O. Walther eds., Springer-Verlag, Berlin, 1994, pp. 1-24.

[19] A. Fonda and J. Mawhin, Multiple periodic solutions of conservative systems with periodic nonlinearity. In: Differential Equations and Applications, Proc. Int. Conf. Columbus, Ohio, Vol. I, A.R. Aftabizadeh ed., Ohio Univ. Press, Athens, OH., 1989, pp. 298-304. MR 91d:58039 
[20] K. Gęba and A. Granas, Algebraic topology in linear normed spaces I-V, Bull. Acad. Polon. Sci., I: 13 (1965), 287-290; II: 13 (1965), 341-346; III: 15 (1967), 137-143; IV: 15 (1967), 145-152; V: 17 (1969), 123-130. MR 31:1708; MR 32:4694; MR 35:6141; MR 35:6142; MR 39:7592

[21] _ Infinite dimensional cohomology theories, J. Math. Pure Appl. 52 (1973), 145-270. MR 52:1762

[22] D. Gromoll and W. Meyer, On differentiable functions with isolated critical points, Topology 8 (1969), 361-369. MR 39:7633

[23] J. Hulshof and R. van der Vorst, Differential systems with strongly indefinite variational structure, J. Func. Anal. 114 (1993), 32-58. MR 94g:35073

[24] W. Kryszewski, B. Przeradzki and S. Wereński, Remarks on approximation methods in degree theory, Trans. Amer. Math. Soc. 316 (1989), 97-114. MR 90b:47098

[25] S.J. Li and J.Q. Liu, Some existence theorems on multiple critical points and their applications, Kexue Tongbao 17 (1984), 1025-1027 (in Chinese).

[26] _ , Morse theory and asymptotic linear Hamiltonian system, J. Diff. Eq. 78 (1989), 53-73. MR 90d:58041

[27] S.J. Li and A. Szulkin, Periodic solutions of an asymptotically linear wave equation, Topol. Meth. Nonl. Anal. 1 (1993), 211-230. MR 94i:35016

[28] _ Periodic solutions for a class of nonautonomous Hamiltonian systems, J. Diff. Eq. 112 (1994), 226-238. MR 95g:58038

[29] S.J. Li and M. Willem, Applications of local linking to critical point theory, J. Math. Anal. Appl. 189 (1995), 6-32. MR 96a:58045

[30] J.Q. Liu, A generalized saddle point theorem, J. Diff. Eq. 82 (1989), 372-385. MR 90k:58035

[31] N.G. Lloyd, Degree Theory, Cambridge University Press, Cambridge, 1978. MR 58:12558

[32] Y. Long, Maslov-type index, degenerate critical points, and asymptotically linear Hamiltonian systems, Science in China (Series A) 33 (1990), 1409-1419. MR 92d:58171

[33] Y. Long and E. Zehnder, Morse-theory for forced oscillations of asymptotically linear Hamiltonian systems. In: Stochastic Processes, Physics and Geometry, S. Albeverio, G. Casati, U. Cattaneo, D. Merlini and A. Moresi eds., World Sci. Publ., Singapore, 1990, pp. 528-563. MR 92j:58019

[34] J. Mawhin and M. Willem, Critical Point Theory and Hamiltonian Systems, Springer-Verlag, New York, 1989. MR 90e:58016

[35] W.V. Petryshyn, On the approximation-solvability of equations involving A-proper and pseudo-A-proper mappings, Bull. Amer. Math. Soc. 81 (1975), 223-312. MR 52:9010

[36] — , Approximation-solvability of Nonlinear Functional and Differential Equations, Dekker, New York, 1993. MR 94f:47081

[37] P.H. Rabinowitz, Free vibrations for a semilinear wave equation, Comm. Pure Appl. Math. 31 (1978), 31-68. MR 81i:35109

[38] _ Minimax Methods in Critical Point Theory with Applications to Differential Equations, CBMS Regional Conf. Ser. Math., no. 65, Amer. Math. Soc., Providence, R.I., 1986. MR 87j:58024

[39] E.H. Spanier, Algebraic Topology, McGraw-Hill, New York, 1966. MR 35:1007

[40] A. Szulkin, A relative category and applications to critical point theory for strongly indefinite functionals, Nonl. Anal. TMA 15 (1990), 725-739. MR 91i:58027

[41] _ Cohomology and Morse theory for strongly indefinite functionals, Math. Z. 209 (1992), 375-418. MR 93c:58046

[42] _ , Bifurcation for strongly indefinite functionals and a Liapunov type theorem for Hamiltonian systems, Diff. Int. Eq. 7 (1994), 217-234. MR 94j:58069

[43] K. Tanaka, Infinitely many periodic solutions for the equation: $u_{t t}-u_{x x} \pm|u|^{p-1} u=f(x, t)$. II, Trans. Amer. Math. Soc. 307 (1988), 615-645. MR 89i:35010

[44] J.W. Thomas, Upper and lower bounds for the number of solutions of functional equations involving $k$-set contractions, Rocky Mt. J. Math. 4 (1974), 89-93. MR 48:12180

Department of Mathematics, Nicholas Copernicus University, Toruń, Poland

E-mail address: wkrysz@mat.uni.torun.pl

Department of Mathematics, Stockholm University, Stockholm, Sweden

E-mail address: andrzejs@matematik.su.se 